\title{
WAVE ASYMPTOTICS AT A COSMOLOGICAL TIME-SINGULARITY: CLASSICAL AND QUANTUM SCALAR FIELDS
}

\author{
ALAIN BACHELOT
}

\begin{abstract}
We investigate the propagation of the scalar waves in the FLRW universes beginning with a Big Bang and ending with a Big Crunch, a Big Rip, a Big Brake or a Sudden Singularity. We obtain the sharp description of the asymptotics for the solutions of the linear Klein-Gordon equation, and similar results for the semilinear equation with a subcritical exponent. We prove that the number of cosmological particle creation is finite under general assumptions on the initial Big Bang and the final Big Crunch or Big Brake.
\end{abstract}

\section{INTRODUCTION}

A lot of cosmological models have a time singularity either in the past, such as the Big Bang, or in the future, such as the Big Crunch, the Big Rip introduced by R.R. Caldwell [12], the Big Brake introduced in [33] or a Sudden Singularity (see e.g. [13], 25], 42], for a classification of these time singularities). The physical literature on this subject is very abundant. In particular we mention the works by J. D. Barrow et alii [5], 6], 7], 8]. The non linear hyperbolic equations, mainly the Einstein system, have been deeply investigated near these singularities, we can cite e.g. the work by L. Andersson and A. D. Rendall [3] on the Belinskii-Khalatnikov-Lifshitz asymptotics at the singularities, the study of T. Damour, M. Henneaux, A. D. Rendall, M. Weaver [17], the recent papers by I. Rodianski and J. Speck [53], [54], [55], [59], and the Fuchsian methods that have been applied to the Gowdy spacetimes by S. Kichenassamy and A. D. Rendall [37], [46], F. Beyer and P. G. LeFloch [9], [10]; moreover the asymptotic behaviour of generic vacuum solutions in the $T^{3}$-Gowdy setting is deeply investigated by H. Ringström in [48]. There are also numerous works on the field equations in the smooth (without singulatity) FLRW space-times, in particular in the De Sitter universe (see the fundamental work of H. Friedrich [26], and also e.g. [4], [21], [29], [30], [31], [36], [40], [47], [49], [52], [58], 62]). In contrast, surprisingly enough, few mathematical papers deal with the simpler issue of the behaviour of the linear fields near the cosmological singularities. We can cite the works by P. T. Allen and A. D. Rendall [2] on the cosmological linearized perturbations; there are also recent works: in an important monography [50], H. Ringström investigates the linear systems on cosmological backgrounds, in particular on the Kasner solutions; 51] deals with the Klein-Gordon equation on Bianchi backgrounds and silent singularities; A. Alho, G. Fournodavlos, A. T. Franzen study the wave equation near a Big Bang in [1]. In this paper, we study the linear Klein-Gordon equation $\square u+m^{2} u+\xi R u=0$ on a type of spatially complete warped geometries, including the FLRW universes for which the scale factor has time singularities, and we obtain accurate asymptotics of the fields at these points. These issues are also investigated by H. Ringström who considers in [50] spatially compact settings whith a different methodology. The important case of $\xi=0, m=0$ is studied for some Big Bang in [1] and [51] (see Remark VI.3 below). We also discuss the semilinear equations in the subcritical case and we get similar results. Concerning the linear quantum fields, we investigate the cosmological particle production between a Big Bang and a Big Crunch or a Big Brake. In short, in this paper, we develop the rigorous mathematical framework for most of the results obtained by J. D. Barrow et alii in the previously cited papers. Now we describe our setting and the results. 
Given a $d$-dimensional complete Riemannian $C^{2}$ manifold $\left(\mathbf{K}, \gamma_{i j} d \mathbf{x}^{i} d \mathbf{x}^{j}\right), d \geqslant 3$, and a bounded open interval $I=\left(t_{-}, t_{+}\right) \subset \mathbb{R}$, we consider the FLRW universe $(\mathcal{M}, g)$ where $\mathcal{M}:=I_{t} \times \mathbf{K}_{\mathbf{x}}$ is endowed with the Lorentzian metric

$$
g_{\mu \nu} d x^{\mu} d x^{\nu}=d t^{2}-a^{2}(t) \gamma_{i j} d \mathbf{x}^{i} d \mathbf{x}^{j} .
$$

The scale factor $a(t)$ is a positive function in $C^{2}(I)$ and we assume that near $t_{ \pm}$we have for $k \leqslant 2$

$$
\frac{d^{k} a}{d t^{k}}(t)=\frac{d^{k}}{d t^{k}}\left[c_{0}^{ \pm}\left|t-t_{ \pm}\right|^{\eta_{0}^{ \pm}}+c_{1}^{ \pm}\left|t-t_{ \pm}\right|^{\eta_{1}^{ \pm}}\right]+o\left(\left|t-t_{ \pm}\right|^{\eta_{1}^{ \pm}-k}\right), \quad t \rightarrow t_{ \pm},
$$

where the coefficients $c_{0}^{ \pm}, \eta_{j}^{ \pm} \in \mathbb{R}$ satisfy :

$$
c_{0}^{ \pm}>0, \eta_{0}^{ \pm}<\eta_{1}^{ \pm} .
$$

The Big Bang (resp. the Big Crunch) corresponds to the case $\eta_{0}^{-}>0$ (resp. $\eta_{0}^{+}>0$ ). It will be useful to distinguish the $C^{0}-$ Big Bang/Crunch for which $\eta_{0}^{ \pm} \in(0,1)$, from the $C^{1}-$ Big Bang/Crunch for which $\eta_{0}^{ \pm} \geqslant 1$. A Sudden Singularity at $t_{ \pm}$is associated to $\eta_{0}^{ \pm}=0$ and $\eta_{1}^{ \pm} \notin \mathbb{N}$. A Big Brake is a Sudden Singularity at $t_{+}$with $c_{1}^{+}>0$ and $\eta_{1}^{+}>1$. A very severe singularity is the Big Rip, defined by $\eta_{0}^{ \pm}<0$, that is Slow if $\eta_{0}^{ \pm} \in(-1,0)$ and Strong if $\eta_{0}^{ \pm} \leqslant-1$. In this paper we consider an universe with such time singularities at $t_{ \pm}$, and we investigate the asymptotic behaviour near $t_{ \pm}$of the solution $u$ of the Klein-Gordon type equation

$$
\square_{g} u+m^{2} u+\xi R_{g} u=0,
$$

where $\square_{g}$ is the D'Alembertian operator

$$
\square_{g} u:=\frac{1}{\sqrt{|\operatorname{det}(g)|}} \partial_{\mu}\left(g^{\mu \nu} \sqrt{|\operatorname{det}(g)|} \partial_{\nu} u\right)=\left[\partial_{t}^{2}+d \frac{a^{\prime}(t)}{a(t)} \partial_{t}-\frac{1}{a^{2}(t)} \Delta_{\mathbf{K}}\right] u
$$

$\Delta_{\mathbf{K}}$ is the Laplace-Beltrami operator on $\mathbf{K}$,

$$
\Delta_{\mathbf{K}} u:=\frac{1}{\sqrt{\operatorname{det}(\gamma)}} \partial_{i}\left(\gamma^{i j} \sqrt{\operatorname{det}(\gamma)} \partial_{j} u\right),
$$

$m \geqslant 0$ is the mass of the field, $\xi \in \mathbb{R}$ is a given constant, and $R_{g}$ is the Ricci scalar of $g$. We may consider (I.4) as a kind of master equation arising in different contexts. $u$ can be a component of the metric tensor when the first-order perturbed Einstein equations are recast as a second order PDE, or $u$ is a scalar field involving in several scenarios studied in cosmology, such as the quintessence (see e.g. [5], [7], 8], [14, [24] and the references therein).

In this Klein-Gordon equation, $\xi$ that is associated with the coupling of the field with the geometry, plays a fundamental role. The behaviour of the field near the time singularity $t_{ \pm}$crucially depends on its value. To discuss the different situations it is convenient to use the conformal time $\tau$ that, given $t_{0} \in\left(t_{-}, t_{+}\right)$, is defined by

$$
\tau:=\int_{t_{0}}^{t} \frac{1}{a(s)} d s
$$

and we introduce the Liouville transform $\varphi$ of $u$ :

$$
\varphi(\tau, \mathbf{x}):=[a(t)]^{\frac{d-1}{2}} u(t, \mathbf{x}) .
$$

Then $u$ is solution of (I.4) iff $\varphi$ satisfies

$$
\left\{\partial_{\tau}^{2}-\Delta_{\mathbf{K}}+\xi R_{\gamma}(\mathbf{x})+V(\tau)\right\} \varphi=0, \quad \tau \in\left(\tau_{-}, \tau_{+}\right),
$$

where

$$
V(\tau)=m^{2} \alpha^{2}(\tau)+\left(\xi-\frac{d-1}{4 d}\right) Q(\tau), \quad Q(\tau):=d(d-3) \frac{\alpha^{2}(\tau)}{\alpha^{2}(\tau)}+2 d \frac{\alpha^{\prime \prime}(\tau)}{\alpha(\tau)}
$$




$$
\alpha(\tau):=a(t), \quad \tau_{ \pm}:=\int_{t_{0}}^{t_{ \pm}} \frac{1}{a(s)} d s \in[-\infty, \infty] .
$$

The behavior of the field depends on the asymptotic behavior of the potential near the singularity. We summarize the different cases in Figure 1.

\begin{tabular}{|c|c|c|c|c|}
\hline Singularity & $\eta_{0}^{+}$ & $\xi$ & $\tau_{+}$ & $V(\tau)$ \\
\hline$C^{1}$ Big Crunch & $(1, \infty)$ & $\mathbb{R}$ & $+\infty$ & $O\left(\tau^{-2}\right)$ \\
\hline$C^{1}$ Big Crunch & 1 & $\mathbb{R}$ & $+\infty$ & $C(\xi-(d-1) / 4 d)+O\left(e^{-c_{0}^{+} \tau}\right)$ \\
\hline$C^{0}$ Big Crunch & $(0,1)$ & $\frac{d-1}{4 d}$ & $<\infty$ & $\sim C\left(\tau_{+}-\tau\right)^{2 \eta_{0}^{+} /\left(1-\eta_{0}^{+}\right)}$ \\
\hline$C^{0}$ Big Crunch & $(0,1)$ & $\neq \frac{d-1}{4 d}$ & $<\infty$ & $\sim C\left(\tau_{+}-\tau\right)^{-2}$ \\
\hline Sudden Singularity & 0 & $\frac{d-1}{4 d}$ & $<\infty$ & $\sim m^{2}\left(c_{0}^{+}\right)^{2}$ \\
\hline Sudden Singularity & 0 & $\neq \frac{d-1}{4 d}$ & $<\infty$ & $\sim m^{2}\left(c_{0}^{+}\right)^{2}+C c_{1}^{+}\left(\tau_{+}-\tau\right)^{\eta_{1}^{+}-2}$ \\
\hline Big Rip & $(-\infty, 0)$ & $\frac{d-1}{4 d}$ & $<\infty$ & $\sim C\left(\tau_{+}-\tau\right)^{2 \eta_{0}^{+} /\left(1-\eta_{0}^{+}\right)}$ \\
\hline Big Rip & $(-\infty, 0)$ & $\neq \frac{d-1}{4 d}$ & $<\infty$ & $\sim C\left(\tau_{+}-\tau\right)^{-2}$ \\
\hline
\end{tabular}

Figure 1. Asymptotics of the potential at the singularity. In the last column, $C \in \mathbb{R} \backslash\{0\}$ and $V(\tau) \sim W(\tau)$ means $V(\tau)=W(\tau)+o(W(\tau))$ as $\tau \rightarrow \tau_{+}$.

In the case of a $C^{1}-$ Big Crunch, $\eta_{0}^{+} \geqslant 1$, we have $\tau_{+}=\infty$ and we deal with a scattering problem for a Klein-Gordon equation with a time dependent potential $V(\tau)$. This situation is simple because $V(\tau)=O\left(\tau^{-2}\right)$ if $\eta_{0}^{+}>1$ hence we have to compare the solutions of (I.7) with those of

$$
\left\{\partial_{\tau}^{2}-\Delta_{\mathbf{K}}+\xi R_{\gamma}(\mathbf{x})\right\} \varphi=0, \quad \tau \in[0, \infty) .
$$

If $\eta_{0}^{+}=1, V(\tau)=\left(c_{0}^{+}\right)^{2} d(d-1)\left(\xi-\frac{d-1}{4 d}\right)+O\left(e^{-c_{0}^{+} \tau}\right)$ and the asymptotic dynamics is given by

$$
\left\{\partial_{\tau}^{2}-\Delta_{\mathbf{K}}+\xi R_{\gamma}(\mathbf{x})+\left(c_{0}^{+}\right)^{2} d(d-1)\left(\xi-\frac{d-1}{4 d}\right)\right\} \varphi=0, \quad \tau \in[0, \infty) .
$$

Therefore the investigation of the behaviour of $u(t)$ at the singularity $t=t_{+}$is reduced to a classic problem of asymptotic completeness of wave operators for $\tau \rightarrow+\infty$. Given $u$ solution of (I.4), we look for $\varphi_{0}^{+}$and $\varphi_{1}^{+}$such that the solution $\varphi$ of (I.10) or (I.11) with initial data $\varphi(0)=\varphi_{0}^{+}$, $\partial_{\tau} \varphi(0)=\varphi_{1}^{+}$, satisfies in a suitable sense

$$
[a(t)]^{\frac{d-1}{2}} u(t, \mathbf{x}) \sim \varphi(\tau, \mathbf{x}), \quad a(t) \partial_{t}\left[[a(t)]^{\frac{d-1}{2}} u(t, \mathbf{x})\right] \sim \partial_{\tau} \varphi(\tau, \mathbf{x}), \quad t \rightarrow t_{+} .
$$

Conversely, given $\varphi_{0}^{+}, \varphi_{1}^{+}$, we expect to find $u$ solution of (I.4) satisfying (I.12) and we want to prove that the map

$$
W_{+}:\left(u\left(t_{0}\right), \partial_{t} u\left(t_{0}\right)\right) \mapsto\left(\varphi_{0}^{+}, \varphi_{1}^{+}\right)
$$

is an isomorphism on a suitable functional space. Numerous papers have been devoted to this issue for wave equations with a time dependent mass (see e.g. 20] and the references therein). We show that all the expected results hold with a convenient cut-off for the low frequencies in order to eliminate the exponentially increasing solutions of (I.10) or (I.11). These modes possibly occur if $\xi R_{\gamma}(\mathbf{x})$ is negative at some points. In this case, the spectrum of the operator $-\Delta_{\mathbf{K}}+\xi R_{\gamma}$ can intersect $(-\infty, 0)$ and we need an infrared cut-off to eliminate these solutions.

The case $\eta_{0}^{+}<1$ is deeply different. We have $\tau_{+}<\infty$, therefore we are interested in the existence of the following limits

$$
\varphi_{0}^{+}:=\lim _{t \rightarrow t_{+}}[a(t)]^{\frac{d-1}{2}} u(t, \mathbf{x})=\lim _{\tau \rightarrow \tau_{+}} \varphi(\tau, \mathbf{x})
$$




$$
\varphi_{1}^{+}:=\lim _{t \rightarrow t_{+}} a(t) \partial_{t}\left([a(t)]^{\frac{d-1}{2}} u(t, \mathbf{x})\right)=\lim _{\tau \rightarrow \tau_{+}} \partial_{\tau} \varphi(\tau, \mathbf{x}) .
$$

Moreover $\alpha^{2}(\tau)=O\left(\left(\tau_{+}-\tau\right)^{\gamma}\right)$ with $\gamma=\frac{2 \eta_{0}^{+}}{1-\eta_{0}^{+}}>-2$, and $Q(\tau) \sim c\left(\tau_{+}-\tau\right)^{-2}$. Therefore (I.7) is a Klein-Gordon equation with a time-dependent potential $V(\tau)$ that is singular at $\tau_{+}$. The existence of the previous limits is obtained in extending to $\left[0, \tau_{+}\right]$the solutions defined on $\left[0, \tau_{+}\right)$. Conversely, given $\varphi_{0}^{+}, \varphi_{1}^{+}$, we investigate the Cauchy problem for the singular Klein-Gordon equation (I.7) with the initial data specified at the singularity $\tau_{+}$. Therefore we want to prove the existence of the operator $W_{+}$defined by (I.13) and its invertibility. Such an equation has been investigated by D. Del Santo, T. Kinoshita, M. Reissig in [19], where the authors study the behaviour of the solution near the time singularity of a Klein-Gordon equation. It turns out that their equation corresponds, in our context, to the case for which $\mathbf{K}$ is the Euclidean space. We shall take advantage of the elegant techniques used in [19] to investigate our general situation. We now briefly present our results for the future time singularity $t_{+}$(the results at $t_{-}$are obtained straightforwardly by reversing time).

First we consider the case

$$
\xi=\frac{d-1}{4 d}
$$

called conformal coupling (if the mass is zero we have $V=0$ and the equation (I.4) is conformally invariant). Then the potential $V$ in equation (I.7) is just

$$
V(\tau)=m^{2} \alpha^{2}(\tau),
$$

and we have $V(\tau) \sim c\left(\tau_{+}-\tau\right)^{\gamma}$. If $\eta_{0}^{+}>-1$ we have $\gamma>-1$ hence $V \in L^{1}\left(0, \tau_{+}\right)$. This integrability allows to easily solve the Cauchy problem even for the initial data specified at the singularity, therefore $W_{+}$exists and is invertible. The case of the Strong Big Rip $\eta_{0}^{+} \leqslant-1$ is much more delicate. We prove that $\varphi_{0}^{+}$is well defined but in general $\varphi_{1}^{+}$does not exist as we can see in explicit examples. We summarize our results for the conformal coupling in Figure 2 ,

\begin{tabular}{|c|c|c|c|c|}
\hline Singularity & $\eta_{0}^{+}$ & Infrared Cut - Off & $\varphi_{0}^{+}$ & $\varphi_{1}^{+}$ \\
\hline$C^{1}$ Big Crunch & {$[1, \infty)$} & $Y E S$ & $Y E S$ & $Y E S$ \\
\hline$C^{0}$ Big Crunch & $(0,1)$ & & & \\
Sudden Singularity & 0 & $N O$ & $Y E S$ & $Y E S$ \\
Slow Big Rip & $(-1,0)$ & & & \\
\hline Strong Big Rip & $(-\infty,-1]$ & NO & $Y E S$ & $N O$ \\
\hline
\end{tabular}

Figure 2. Results of existence of $\varphi_{j}^{+}$for the conformal coupling $\xi=\frac{d-1}{4 d}$. When $\varphi_{0}^{+}$and $\varphi_{1}^{+}$exist, $W_{+}$is an isomorphism.

The non conformal coupling corresponds to $\xi \neq \frac{d-1}{4 d}$. In this case the singularity of the potential $V$ at $\tau_{+}$is strong if $\eta_{0}^{+} \neq 0$ since

$$
V(\tau) \sim\left(\xi-\frac{d-1}{4 d}\right)\left(d+1-\frac{2}{\eta_{0}^{+}}\right) d \frac{\left(\eta_{0}^{+}\right)^{2}}{1-\eta_{0}^{+}}\left(\tau_{+}-\tau\right)^{-2}, \quad \tau \rightarrow \tau_{+} .
$$

Let's consider the ordinary differential equation

$$
\varphi^{\prime \prime}+A^{2} \varphi+\frac{\frac{1}{4}-\mu^{2}}{\left(\tau_{+}-\tau\right)^{2}} \varphi=0, \quad \tau<\tau_{+},
$$


that is a toy model for (I.7) (for example if $\mathbf{K}=S^{d}$ ). The solutions are given by

$$
\varphi(\tau)=\left(\tau_{+}-\tau\right)^{\frac{1}{2}}\left[C_{1} J_{\mu}\left(A\left(\tau_{+}-\tau\right)\right)+C_{2} Y_{\mu}\left(A\left(\tau_{+}-\tau\right)\right)\right],
$$

and we can see that in general the limits (I.14) and (I.15) can not exist. Nevertheless, if $\mu^{2}<0$ $\varphi(\tau)$ tends to zero as $\tau \rightarrow \tau_{+}$(and $\partial_{\tau} \varphi(\tau)$ can be diverging). Therefore it is natural to investigate the case

$$
\left(\xi-\frac{d-1}{4 d}\right)\left(d+1-\frac{2}{\eta_{0}^{+}}\right) d \frac{\left(\eta_{0}^{+}\right)^{2}}{1-\eta_{0}^{+}}>\frac{1}{4} .
$$

We prove that in this case, the solution of (I.7) with $\eta_{0}^{+}<1, \eta_{0}^{+} \neq 0$, tends also to zero. This result is not surprising since (1.18) assures that $V$ acts like an infinite potential barrier. The case of the $C^{0}$-Big Crunch without hypothese (I.18) is open but there are recent results for $\xi=0, d=3$, $m=0$ : we have to mention [1] if $\mathbf{K}=\mathbb{R}^{3}, \gamma_{i j}=\delta_{j}^{i}, a(t)=t^{\eta_{0}}, 1 / 3<\eta_{0}<1$, and the general study by H. Ringström [51], for the Bianchi spacetimes with silent singularities (see Remark VI.3 below).

The situation $\eta_{0}^{+}=0$ is very special since $V(\tau)$ is less singular, and the asymptotic behaviour of the field near a Sudden Singularity heavily depends on the lower term associated with $\eta_{1}^{+}$.

\begin{tabular}{|c|c|c|c|c|c|c|}
\hline Singularity & $\eta_{0}^{+}$ & $\eta_{1}^{+}$ & $\xi$ & Infrared Cut - Off & $\varphi_{0}^{+}$ & $\varphi_{1}^{+}$ \\
\hline$C^{1}$ Big Crunch & {$[1, \infty)$} & $\left(\eta_{0}^{+}, \infty\right)$ & $\mathbb{R}$ & $Y E S$ & $Y E S$ & $Y E S$ \\
\hline$C^{0}$ Big Crunch & $(0,1)$ & $\left(\eta_{0}^{+}, \infty\right)$ & $(\underline{I .18})$ & $N O$ & $Y E S(=0)$ & $N O$ \\
\hline Big Brake & 0 & $(1, \infty)$ & $\mathbb{R}$ & $N O$ & $Y E S$ & $Y E S$ \\
\hline Sudden Singularity & 0 & $(0,1)$ & $\mathbb{R}$ & $N O$ & $Y E S$ & $N O$ \\
\hline Big Rip & $(-\infty, 0)$ & $\left(\eta_{0}^{+}, \infty\right)$ & $(\underline{I .18})$ & $N O$ & $Y E S(=0)$ & $N O$ \\
\hline
\end{tabular}

Figure 3. Results of existence of $\varphi_{j}^{+}$for non conformal coupling $\xi \neq \frac{d-1}{4 d}$. When $\varphi_{0}^{+}$and $\varphi_{1}^{+}$exist, $W_{+}$is an isomorphism.

We also tackle the problem of nonlinear fields. Important results are known for smooth universes, in particular for the De Sitter spacetime. Besides the studies on the stability of the Einsteinnonlinear field systems [47], 49], [52], 58], 62], we can cite for the analysis of non-linear wave equations, the works by Ebert and Reissig [21], Galstian and Yagdjian [30], [31], Nakamura [40]. At our knowledge a lot of hard issues about interacting fields on singular Lorentzian manifolds remain to be investigated. In this paper we present just a modest contribution to this topic. We consider the semilinear Klein-Gordon equation

$$
\square_{g} u+m^{2} u+\xi R_{g} u=-C u|u|^{p-1} .
$$

In this work we investigate only the subcritical case

$$
1 \leqslant p \leqslant \frac{d}{d-2} .
$$

In this simple situation we may use the usual Sobolev embeddings to solve the Cauchy problem, and choosing $C>0$ the finite energy solutions are global. We study the asymptotic behaviour at a Big Bang/Crunch or at a Sudden Singularity for the conformal coupling $\xi=\frac{d-1}{4 d}$ and we obtain the same results as for the linear equation.

Finally we investigate some quantum aspects in a universe beginning with a Big Bang and ending with a Big Crunch or a Sudden Singularity. The study of cosmological particle creation in an expanding universe has a long history since the $\mathrm{PhD}$ of L. Parker in 1965 [44]. We have to mention the work of S. A. Fulling [28] for the spatially flat Robertson-Walker metrics and the 
generalized Kasner (Bianchi type I) metrics. For more recent works, see e.g. [11, [18], [27], [34]. Fulling had investigated the creation of particles between two regular times $t_{1}, t_{2} \in\left(t_{-}, t_{+}\right)$on $\mathbf{K}=\mathbb{R}^{3}$. Here we consider the case of the time singularities $t_{1}=t_{-}, t_{2}=t_{+}$when a Big Bang, a Big Crunch or a Big Brake occurs and $\mathbf{K}$ is a $d$-dimensional compact manifold. In the last part, we give sufficient conditions on $d$ and $\eta_{j}^{ \pm}$for the number of cosmological particle creation to be finite. This number $\mathcal{N}$ is estimated by the Zeta function of the Laplacian on $\mathbf{K}$,

$$
\mathcal{N} \lesssim \sum_{n} \lambda_{n}^{-l(d)-1}, \quad l(d):=\max (2,[d / 2]),
$$

where $[x]$ is the integer part of $x$ and $\lambda_{n}$ are the positive eigenvalues of $-\Delta_{\mathbf{K}}$. The proof, inspired from Fulling [28, is based on the Liouville-Green (or WKB) approximation that needs a sufficient regularity of the coefficients. That explains why the results are established for a scale factor $a \in C^{l}\left(t_{-}, t_{+}\right)$satisfying (I.2) for $0 \leqslant k \leqslant l$ with $l=l(d)$ if $\xi=\frac{d-1}{4 d}$ and $l=l(d)+2$ if $\xi \neq \frac{d-1}{4 d}$.

\section{The Linear and Nonlinear Global Cauchy Problems}

In this part we investigate the Cauchy problem associated to the inhomogeneous Klein-Gordon equation

$$
\begin{gathered}
\left\{\partial_{t}^{2}+d \frac{a^{\prime}(t)}{a(t)} \partial_{t}-\frac{1}{a^{2}(t)} \Delta_{\mathbf{K}}+m^{2}+\xi R_{g}(t, \mathbf{x})\right\} u=F, \quad t \in I, \quad \mathbf{x} \in \mathbf{K}, \\
u\left(t_{0}, \mathbf{x}\right)=u_{0}(\mathbf{x}), \quad \partial_{t} u\left(t_{0}, \mathbf{x}\right)=u_{1}(\mathbf{x}),
\end{gathered}
$$

where $t_{0}$ is fixed in $I$. If $a \in C^{\infty}(I)$, the existence and the uniqueness of the solution is well known since the seminal works of Leray [38], for smooth or distributions initial data. We denote $X$ the space $C^{\infty}(\mathbf{K})$ or $C_{0}^{\infty}(\mathbf{K})$ or $\mathcal{D}^{\prime}(\mathbf{K})$. Then for any $u_{0}, u_{1} \in X, F \in C^{\infty}(I ; X)$, the classic results of Leray assure the existence and uniqueness of $u \in C^{\infty}(I ; X)$. Here we are interested in the finite energy solutions and the quantum fields, and we assume just $a \in C^{2}(I)$. Hence we have to introduce a suitable Hilbertian functional framework (for the $H^{1}$-solutions see [15]). If $\xi \neq 0$ we assume that the scalar curvature $R_{\gamma}$ of $(\mathbf{K}, \gamma)$ is bounded

$$
R_{\gamma} \in L^{\infty}(\mathbf{K})
$$

We denote $L^{2}(\mathbf{K})$ the $L^{2}$-Lebesgue space on $\mathbf{K}$ endowed with the volume measure associated to the metric $\gamma$, that we simply denote $d \mathbf{x}$. A well known result by Strichartz (Theorem 2.4 in 61]) assures that $\Delta_{\mathbf{K}}$ is essentially selfadjoint on $C_{0}^{\infty}(\mathbf{K})$ since $\mathbf{K}$ is a complete manifold, and we shall use the Sobolev spaces $H^{s}(\mathbf{K}), s \in \mathbb{R}$, defined as the closure of $C_{0}^{\infty}(\mathbf{K})$ for the norm

$$
\|f\|_{H^{s}(\mathbf{K})}:=\left\|\left(-\Delta_{\mathbf{K}}+1\right)^{\frac{s}{2}} f\right\|_{L^{2}(\mathbf{K})} .
$$

More precisely, $H^{s}(\mathbf{K})$ is the Bessel-potential space introduced by Strichartz [61]. Alternatively, if $\mathbf{K}$ has a bounded geometry, we can characterize $H^{s}(\mathbf{K})$ via localization and pull-back onto $\mathbb{R}^{d}$ (see [35] and the references therein). Moreover, if $s$ is a positive integer, Triebel proved that $H^{s}(\mathbf{K})$ is just the classical Sobolev space $W^{s}(\mathbf{K})$ introduced by Aubin, which contains all $L^{2}$ functions on $\mathbf{K}$ having bounded covariant derivatives up to order $s$. The following Proposition states that the Cauchy problem is well posed in these spaces. These expected results are not surprising but due to the weak regularity of the coefficients they are not straight consequences of well known results on the problems of evolution with time dependent Hamiltonians and smooth coefficients (see e.g. [63]). To overcome this difficulty we combine the variational approach of Lions [39] and the Picard iteration method.

Proposition II.1. Let a be a strictly positive function in $C^{2}(I)$ and $m \geqslant 0$. If $\xi \neq 0$, we assume that (II.3) holds. Given $\theta \in[0,1], u_{0} \in H^{1-\theta}(\mathbf{K}), u_{1} \in H^{-\theta}(\mathbf{K}), F \in L_{l o c}^{2}\left(I ; H^{-\theta}(\mathbf{K})\right)$, there exists a unique $u \in C^{0}\left(I ; H^{1-\theta}(\mathbf{K})\right) \cap C^{1}\left(I ; H^{-\theta}(\mathbf{K})\right)$ solution of (II.1) satisfying (II.2). Moreover 
$\left(u_{0}, u_{1}, F\right) \mapsto u$ is a continuous mapping of these spaces: for any $\left[t_{-}^{\prime}, t_{+}^{\prime}\right] \subset I$, there exists $C>0$ such that for all $t \in\left[t_{-}^{\prime}, t_{+}^{\prime}\right]$ we have :

$$
\|u(t)\|_{H^{1-\theta}}^{2}+\left\|\partial_{t} u(t)\right\|_{H^{-\theta}}^{2} \leqslant C\left(\left\|u_{0}\right\|_{H^{1-\theta}}^{2}+\left\|u_{1}\right\|_{H^{-\theta}}^{2}+\left|\int_{t_{0}}^{t}\|F(s)\|_{H^{-\theta}}^{2} d s\right|\right) .
$$

If $u$ and $\tilde{u}$ are two solutions with $\theta=\frac{1}{2}$ and $F=0$, then

$$
\sigma(u, \tilde{u}):=[a(t)]^{d}\left(\left\langle u(t), \partial_{t} \tilde{u}(t)\right\rangle-\left\langle\tilde{u}(t), \partial_{t} u(t)\right\rangle\right)
$$

does not depend on $t$, where $\langle$,$\rangle denotes the duality bracket between H^{\frac{1}{2}}(\mathbf{K})$ and $H^{-\frac{1}{2}}(\mathbf{K})$.

Proof. It is convenient to eliminate the first order time derivative in (II.1) by putting

$$
v(t, \mathbf{x}):=[a(t)]^{\frac{d}{2}} u(t, \mathbf{x}) .
$$

Then $u$ is solution of (II.1) iff $v$ satisfies

$$
\left\{\partial_{t}^{2}-\frac{1}{a^{2}(t)} \Delta_{\mathbf{K}}+m^{2}+\xi R_{g}+\frac{d}{2}\left[\frac{a^{\prime 2}(t)}{a^{2}(t)}\left(1-\frac{d}{2}\right)-\frac{a^{\prime \prime}(t)}{a(t)}\right]\right\} v=[a(t)]^{\frac{d}{2}} F .
$$

Given $\left[t_{-}^{\prime}, t_{+}^{\prime}\right] \subset I$ with $t_{0} \in\left[t_{-}^{\prime}, t_{+}^{\prime}\right]$, we solve the Cauchy problem for $v$ on this interval in the same spaces $H^{1-\theta}, H^{-\theta}, L_{l o c}^{2}\left(I ; H^{-\theta}\right)$. First we compute the Ricci curvature for the metric $g$,

$$
R_{g}(t, \mathbf{x})=\frac{1}{a^{2}(t)} R_{\gamma}(\mathbf{x})+2 d \frac{a^{\prime \prime}(t)}{a(t)}+d(d-1) \frac{a^{\prime 2}(t)}{a^{2}(t)}
$$

hence (II.7) becomes

$$
\left\{\partial_{t}^{2}-\frac{1}{a^{2}(t)} \Delta_{\mathbf{K}}+m^{2}+\frac{\xi}{a^{2}(t)} R_{\gamma}(\mathbf{x})+B(t)\right\} v=[a(t)]^{\frac{d}{2}} F
$$

where

$$
B(t):=\xi\left[2 d \frac{a^{\prime \prime}(t)}{a(t)}+d(d-1) \frac{a^{2}(t)}{a^{2}(t)}\right]+\frac{d}{2}\left[\frac{a^{\prime 2}(t)}{a^{2}(t)}\left(1-\frac{d}{2}\right)-\frac{a^{\prime \prime}(t)}{a(t)}\right] \in C^{0}(I) .
$$

Because of the weak regularity of $B$ we cannot apply general results to assure the existence and the uniqueness of the solution, and we consider an intermediate problem. We remark that the family of sesquilinear forms on $H^{1}(\mathbf{K})$

$$
A(t ; v, w):=\int_{\mathbf{K}}\left\{\frac{1}{a^{2}(t)} \nabla_{\mathbf{K}} v \cdot \overline{\nabla_{\mathbf{K}} w}+\left(m^{2}+\frac{\xi}{a^{2}(t)} R_{\gamma}\right) v \bar{w}\right\} d \mathbf{x}
$$

satisfies assumptions (8.1), (8.2) p. 265 and (9.37) p. 290 of [39]. Therefore the Cauchy problem

$$
\left\{\partial_{t}^{2}-\frac{1}{a^{2}(t)} \Delta_{\mathbf{K}}+m^{2}+\frac{\xi}{a^{2}(t)} R_{\gamma}(\mathbf{x})\right\} v=G
$$

is well-posed on $\left[t_{-}^{\prime}, t_{+}^{\prime}\right]$ for $v\left(t_{0}\right) \in H^{1-\theta}, \partial_{t} v\left(t_{0}\right) \in H^{-\theta}, G \in L^{2}\left(\left[t_{-}^{\prime}, t_{+}^{\prime}\right] ; H^{-\theta}\right)$ : this a direct consequence of the following results of chapter 3 in [39]: Theorem 8.2 if $\theta=0$, Theorem 9.4 if $\theta=1$, Theorem 9.5 if $0<\theta<1$. In particular the finite energy solutions of (II.11) satisfy the energy inequality : there exists $C>0$ such for any $t \in\left[t_{-}^{\prime}, t_{+}^{\prime}\right]$ we have

$$
\|v(t)\|_{H^{1}}^{2}+\left\|\partial_{t} v(t)\right\|_{L^{2}}^{2} \leqslant C\left(\left\|v\left(t_{0}\right)\right\|_{H^{1}}^{2}+\left\|\partial_{t} v\left(t_{0}\right)\right\|_{L^{2}}^{2}+\left|\int_{t_{0}}^{t}\|G(s)\|_{L^{2}}^{2} d s\right|\right) .
$$

Furthermore, taking advantage of the peculiar structure of (II.11), we can show that a similar estimate for weaker solutions of (II.11) holds. If $v \in C^{0}\left(I ; H^{1-\theta}\right) \cap C^{1}\left(I ; H^{-\theta}\right)$ is a solution of 
(II.11) with $G \in L_{l o c}^{2}\left(I ; H^{-\theta}\right)$, we put $\tilde{v}:=\left(-\Delta_{\mathbf{K}}+1\right)^{-\frac{\theta}{2}} v$. Applying (II.12) with $\xi=0$ to $\tilde{v}$ we get

$$
\begin{gathered}
\|v(t)\|_{H^{1-\theta}}^{2}+\left\|\partial_{t} v(t)\right\|_{H^{-\theta}}^{2} \leqslant C\left(\left\|v\left(t_{0}\right)\right\|_{H^{1-\theta}}^{2}+\left\|\partial_{t} v\left(t_{0}\right)\right\|_{H^{-\theta}}^{2}+\left|\int_{t_{0}}^{t}\|\tilde{G}(s)\|_{L^{2}}^{2} d s\right|\right), \\
\tilde{G}:=\left(-\Delta_{\mathbf{K}}+1\right)^{-\frac{\theta}{2}}\left[G-\frac{\xi}{a^{2}(t)} R_{\gamma} v\right] .
\end{gathered}
$$

Since we have

$$
\|\tilde{G}\|_{L^{2}}^{2} \leqslant 2\left(\|G\|_{H^{-\theta}}^{2}+\left\|\frac{\xi}{a^{2}(t)} R_{\gamma} v\right\|_{L^{2}}^{2}\right) \leqslant C^{\prime}\left(\|G\|_{H^{-\theta}}^{2}+\|v\|_{L^{2}}^{2}\right) \leqslant C^{\prime}\left(\|G\|_{H^{-\theta}}^{2}+\|v\|_{H^{1-\theta}}^{2}\right),
$$

we deduce using Gronwall's lemma that the solutions of (II.11) satisfy :

$$
\|v(t)\|_{H^{1-\theta}}^{2}+\left\|\partial_{t} v(t)\right\|_{H^{-\theta}}^{2} \leqslant C^{\prime \prime}\left(\left\|v\left(t_{0}\right)\right\|_{H^{1-\theta}}^{2}+\left\|\partial_{t} v\left(t_{0}\right)\right\|_{H^{-\theta}}^{2}+\left|\int_{t_{0}}^{t}\|G(s)\|_{H^{-\theta}}^{2} d s\right|\right) .
$$

Now we return to the initial value problem for (II.7) by using the Picard method. We solve iteratively

$$
\begin{gathered}
\left\{\partial_{t}^{2}-\frac{1}{a^{2}(t)} \Delta_{\mathbf{K}}+m^{2}+\frac{\xi}{a^{2}(t)} R_{\gamma}(\mathbf{x})\right\} v_{0}=[a(t)]^{\frac{d}{2}} F, \quad v_{0}\left(t_{0}\right)=v\left(t_{0}\right), \partial_{t} v_{0}\left(t_{0}\right)=\partial_{t} v\left(t_{0}\right), \\
\left\{\partial_{t}^{2}-\frac{1}{a^{2}(t)} \Delta_{\mathbf{K}}+m^{2}+\frac{\xi}{a^{2}(t)} R_{\gamma}(\mathbf{x})\right\} v_{k+1}=-B(t) v_{k}, \quad v_{k+1}\left(t_{0}\right)=\partial_{t} v_{k+1}\left(t_{0}\right)=0, k \geqslant 0 .
\end{gathered}
$$

We put

$$
M^{2}:=C\left(\left\|v\left(t_{0}\right)\right\|_{H^{1-\theta}}^{2}+\left\|\partial_{t} v\left(t_{0}\right)\right\|_{H^{-\theta}}^{2}+\int_{t_{-}^{\prime}}^{t_{+}^{\prime}}[a(t)]^{d}\|F(t)\|_{H^{-\theta}}^{2} d t\right) .
$$

Using inequality (II.13), we easily prove by recurrence that

$$
\left\|v_{k}(t)\right\|_{H^{1-\theta}}^{2}+\left\|\partial_{t} v_{k}(t)\right\|_{H^{-\theta}}^{2} \leqslant M^{2} \frac{\left(C\|B\|_{L^{\infty}\left(t_{-}^{\prime}, t_{+}^{\prime}\right)}^{2}\left(t-t_{0}\right)\right)^{k}}{k !} .
$$

Then we conclude that

$$
v(t, \mathbf{x}):=\sum_{k=0}^{\infty} v_{k}(t, \mathbf{x})
$$

is a solution in $C^{0}\left(\left[t_{-}^{\prime}, t_{+}^{\prime}\right] ; H^{1-\theta}\right) \cap C^{1}\left(\left[t_{-}^{\prime}, t_{+}^{\prime}\right] ; H^{-\theta}\right)$ of the initial value problem associated to (II.7). Moreover the continuous dependence with respect to the data is given by the inequality

$$
\sup _{t \in\left[t_{-}^{\prime}, t_{+}^{\prime}\right]}\|v(t)\|_{H^{1-\theta}}+\left\|\partial_{t} v(t)\right\|_{H^{-\theta}} \leqslant C^{\prime} M
$$

where $C^{\prime}>0$ only depends on the interval $\left[t_{-}^{\prime}, t_{+}^{\prime}\right]$. To establish the uniqueness of the solutions of (II.1) and the energy type estimate (II.5), we apply (II.13) with $G(t)=[a(t)]^{\frac{d}{2}} F-B(t) v$, and we deduce using Gronwall's lemma that the solutions of (II.7) satisfy

$$
\|v(t)\|_{H^{1-\theta}}^{2}+\left\|\partial_{t} v(t)\right\|_{H^{-\theta}}^{2} \leqslant C\left(\left\|v\left(t_{0}\right)\right\|_{H^{1-\theta}}^{2}+\left\|\partial_{t} v\left(t_{0}\right)\right\|_{H^{-\theta}}^{2}+\left|\int_{t_{0}}^{t}\|F(s)\|_{H^{-\theta}}^{2} d s\right|\right), \quad t \in\left[t_{-}^{\prime}, t_{+}^{\prime}\right],
$$

for some $C>0$ depending on $t_{ \pm}^{\prime}$. Replacing $v$ by $a^{\frac{d}{2}} u$ we obtain (II.5) with another suitable constant $C$.

To establish (II.6) it is sufficient to consider the case $\theta=1$ thanks to the density of $H^{1}$ in $H^{\frac{1}{2}}$, and the continuous dependence of $u$ with respect the initial data. In this case we have $u, \tilde{u} \in C^{0}\left(I ; H^{1}(\mathbf{K})\right) \cap C^{1}\left(I ; L^{2}(\mathbf{K})\right) \cap C^{2}\left(I ; H^{-1}(\mathbf{K})\right)$. Hence we may compute the time derivative of $\sigma(u(t), \tilde{u}(t))$ and using (III.1) we get that it is equal to zero.

Q.E.D. 
We introduce the propagator $T\left(t, t_{0}\right)$ associated to the linear equation (II.1) with $F=0$, and defined by $T\left(t, t_{0}\right)\left(u_{0}, u_{1}\right):=\left(u(t), \partial_{t} u(t)\right)$ where $u$ is the solution of (II.1) satisfying (II.2). The previous proposition assures that

$$
T \in L_{l o c}^{\infty}\left(I \times I ; \mathcal{L}\left(H^{1-\theta}(\mathbf{K}) \times H^{-\theta}(\mathbf{K})\right)\right), \quad \theta \in[0,1],
$$

and the unique solution of the inhomogenous equation with Cauchy data $\left(u_{0}, u_{1}\right)$ at $t_{0}$ is given by the usual Duhamel formula

$$
\left(u(t), \partial_{t} u(t)\right)=T\left(t, t_{0}\right)\left(u_{0}, u_{1}\right)+\int_{t_{0}}^{t} T(t, s)(0, F(s)) d s .
$$

It will be useful to have a result on the existence of solutions in higher order Sobolev spaces, in particular to treat the non linear equation. We strengthen the assumptions concerning the metric $\gamma$ by assuming that :

$$
\forall \alpha \in \mathbb{N}^{d}, \quad D^{\alpha} \gamma_{i j} \in L^{\infty}(\mathbf{K}), \quad \gamma^{i j} \in L^{\infty}(\mathbf{K}),
$$

where $D$ represents coordinate derivatives in any normal coordinate system. Some equivalent or sufficient conditions have been obtained by J. Eichhorn (Corollary 2.2, Proposition 2.3 and Theorem 2.4 of $[23]$ ).

Proposition II.2. We assume that $(\mathbf{K}, \gamma)$ is a $C^{\infty}$ manifold that satisfies (II.16). Then, given $s \geqslant 0, u_{0} \in H^{s}(\mathbf{K}), u_{1} \in H^{s-1}(\mathbf{K}), F \in L_{l o c}^{2}\left(I ; H^{s-1}(\mathbf{K})\right)$, there exists a unique $u \in C^{0}\left(I ; H^{s}(\mathbf{K})\right) \cap$ $C^{1}\left(I ; H^{s-1}(\mathbf{K})\right)$ solution of (II.1) satisfying (II.2). Moreover $\left(u_{0}, u_{1}, F\right) \mapsto u$ is a continuous mapping of these spaces.

Proof. First we consider the case $s \in \mathbb{N}$. We write $s=2 N+1-\theta$ with $N \in \mathbb{N}, \theta \in\{0,1\}$. Proposition $\llbracket$ II.1 assures the existence of the solution $u \in C^{0}\left(I ; H^{1-\theta}(\mathbf{K})\right) \cap C^{1}\left(I ; H^{-\theta}(\mathbf{K})\right)$. Hence it is sufficient to establish that $u_{N}:=\left(-\Delta_{\mathbf{K}}+1\right)^{N} u$ belongs to the same space. $u_{N}$ is a solution of (I.4) where $F$ is replaced by $\left[\left(-\Delta_{\mathbf{K}}+1\right)^{N}, R_{\gamma}\right] u+\left(-\Delta_{\mathbf{K}}+1\right)^{N} F$. Now a theorem of Eichhorn 22 assures that all the covariant derivatives of the Riemannian curvature tensor are bounded iff (II.16) is satisfied. Therefore (II.16) implies that for any $\alpha \in \mathbb{N}^{d}, \nabla^{\alpha} R_{\gamma} \in L^{\infty}(\mathbf{K})$ and then the commutator $\left[\left(-\Delta_{\mathbf{K}}+1\right)^{N}, R_{\gamma}\right]$ is a bounded operator from $H^{\sigma}(\mathbf{K})$ to $H^{\sigma-2 N+1}(\mathbf{K})$. Now the result for any integer follows from Proposition \I.1 by recurrence on $N$. In the general case with $s \in \mathbb{R}^{+} \backslash \mathbb{N}$ we write $s=N+\theta, \theta \in(0,1)$. The previous result assures that for any $t \in I$, the map $\left(u_{0}, u_{1}, F\right) \longmapsto\left(u(t), \partial_{t} u(t)\right)$ is continuous from $H^{N} \times H^{N-1} \times L_{l o c}^{2}\left(I ; H^{N-1}\right)$ to $H^{N} \times H^{N-1}$, and from $H^{N+1} \times H^{N} \times L_{l o c}^{2}\left(I ; H^{N}\right)$ to $H^{N+1} \times H^{N}$. Since these spaces are interpolation spaces, we deduce from the interpolation thorem (see Theorem 5.1 in [39]) that this map is continuous from $H^{s} \times H^{s-1} \times L_{l o c}^{2}\left(I ; H^{s-1}\right)$ to $H^{s} \times H^{s-1}$. Moreover, we get from the theorem of interpolation of a family of operators (see Theorem 5.2 in [39]) that $u \in C^{0}\left(I ; H^{s}\right) \cap C^{1}\left(I ; H^{s-1}\right)$. Finally the Banach-Steinhauss theorem implies that the map $\left(u_{0}, u_{1}, F\right) \longmapsto\left(u, \partial_{t} u\right)$ is continuous from $H^{s} \times H^{s-1} \times L_{l o c}^{2}\left(I ; H^{s-1}\right)$ to $C^{0}\left(I ; H^{s}\right) \cap C^{1}\left(I ; H^{s-1}\right)$.

Q.E.D.

Finally we consider the global Cauchy problem for the semilinear Klein-Equation (II.1) where $F$ is the subcritical nonlinearity

$$
F(u)=-C u|u|^{p-1}, \quad 1 \leqslant p \leqslant \frac{d}{d-2}, \quad C \geqslant 0 .
$$

To be able to use Sobolev embedding we have to be strengthened the assumptions on the metric. We recall that $(\mathbf{K}, \gamma)$ is a $C^{\infty}$ bounded geometry manifold, if the following conditions are satisfied: (1) the injectivity radius is strictly positive, (2) every covariant derivative of the Riemannian curvature tensor is bounded, i.e., thanks to the Eichhorn theorem, (II.16) is satisfied. 
Theorem II.3. We assume that $(\mathbf{K}, \gamma)$ is a $C^{\infty}$ bounded geometry manifold. Given $u_{0} \in H^{1}(\mathbf{K})$, $u_{1} \in L^{2}(\mathbf{K})$, there exists a unique $u \in C^{0}\left(I ; H^{1}(\mathbf{K})\right) \cap C^{1}\left(I ; L^{2}(\mathbf{K})\right)$ solution of (II.1), (II.17), (II.2). Moreover, there exists a function $K \in C^{0}\left(I^{2} \times \mathbb{R}^{4}\right)$ such that for any solutions $u$ and $\hat{u}$, we have for all $t, t_{0} \in I$,

(II.18)

$$
\begin{aligned}
& \|u(t)-\hat{u}(t)\|_{H^{1}}+\left\|\partial_{t} u(t)-\partial_{t} \hat{u}(t)\right\|_{L^{2}} \\
& \quad \leqslant K\left(t, t_{0},\left\|u\left(t_{0}\right)\right\|_{H^{1}},\left\|\partial_{t} u\left(t_{0}\right)\right\|_{L^{2}},\left\|\hat{u}\left(t_{0}\right)\right\|_{H^{1}},\left\|\hat{u}\left(t_{0}\right)\right\|_{L^{2}}\right)\left(\left\|u\left(t_{0}\right)-\hat{u}\left(t_{0}\right)\right\|_{H^{1}}+\left\|\partial_{t} u\left(t_{0}\right)-\partial_{t} \hat{u}\left(t_{0}\right)\right\|_{L^{2}}\right) .
\end{aligned}
$$

Proof. Due to the assumption on $(\mathbf{K}, \gamma)$, the Sobolev embeddings hold and in particular we have:

$$
H^{1}(\mathbf{K}) \subset L^{\frac{2 d}{d-2}}(\mathbf{K}) .
$$

We deduce that there exists $c_{p}>0$ such that for any $u, \hat{u} \in H^{1}(\mathbf{K})$,

$$
\|F(u)-F(\hat{u})\|_{L^{2}} \leqslant c_{p}\|u-\hat{u}\|_{H^{1}}\left(\|u\|_{H^{1}}+\|\hat{u}\|_{H^{1}}\right)^{p-1} .
$$

Now the proof of the local existence is a classic routine. Given the Cauchy data $\left(u_{0}, u_{1}\right)$ at $t_{0}$, we consider the nonlinear operator $\mathcal{F}$ defined for $(u, v) \in C^{0}\left(I ; H^{1} \times L^{2}\right)$ by

$$
\mathcal{F}(u, v)(t):=T\left(t, t_{0}\right)\left(u_{0}, u_{1}\right)-\int_{t_{0}}^{t} T(t, s)\left(0, C u(s)|u(s)|^{p-1}\right) d s .
$$

Thanks to (II.14) and (II.19), we have for $t \in\left[t_{0}-\epsilon, t_{0}+\epsilon\right]$

$$
\begin{gathered}
\|\mathcal{F}(u, v)(t)\|_{H^{1} \times L^{2}} \leqslant M_{\epsilon}\left(R+\epsilon C c_{p}\|(u, v)\|_{\epsilon}^{p}\right), \\
\|\mathcal{F}(u, v)(t)-\mathcal{F}(\hat{u}, \hat{v})(t)\|_{H^{1} \times L^{2}} \leqslant \epsilon M_{\epsilon} C c_{p}\left(\|(u, v)\|_{\epsilon}+\|(\hat{u}, \hat{v})\|_{\epsilon}\right)^{p-1}\|(u, v)-(\hat{u}, \hat{v})\|_{\epsilon},
\end{gathered}
$$

where $M_{\epsilon}:=\sup _{t, t^{\prime} \in\left[t_{0}-\epsilon, t_{0}+\epsilon\right]}\left\|T\left(t, t^{\prime}\right)\right\|_{\mathcal{L}\left(H^{1} \times L^{2}\right)}, R:=\left\|\left(u_{0}, u_{1}\right)\right\|_{H^{1} \times L^{2}}$, and

$$
\|(u, v)\|_{\epsilon}:=\sup _{t \in\left[t_{0}-\epsilon, t_{0}+\epsilon\right]}\|(u(t), v(t))\|_{H^{1} \times L^{2}} .
$$

We deduce that for $\epsilon>0$ small enough so that

$$
\epsilon C c_{p}\left(2 M_{\epsilon} R\right)^{p} \leqslant R,
$$

then $\mathcal{F}$ is defined from $B_{\epsilon}\left(R M_{\epsilon}\right)$ into itself, where

$$
B_{\epsilon}(\rho):=\left\{(u, v) \in C^{0}\left(\left[t_{0}-\epsilon, t_{0}+\epsilon\right] ; H^{1} \times L^{2}\right), \quad\left\|(u, v)-T\left(., t_{0}\right)\left(u_{0}, u_{1}\right)\right\|_{\epsilon} \leqslant \rho\right\} .
$$

Furthermore, if we also choose $\epsilon>0$ small enough so that

$$
\epsilon C c_{p}(4 R)^{p-1} M_{\epsilon}^{p}<1
$$

then $\mathcal{F}$ is a strict contraction on $B_{\epsilon}\left(R M_{\epsilon}\right)$. The unique fixed point $(u, v)$ in this space satisfies $v=\partial_{t} u$ and $u$ is a solution of (II.1), (II.17), (II.2) on $\left[t_{0}-\epsilon, t_{0}+\epsilon\right]$.

To obtain global existence, we recall an abstract result of Strauss ([60], Theorem 4.1). If $u \in$ $C^{0}\left([0, T] ; H^{1}\right) \cap C^{1}\left([0, T] ; L^{2}\right)$ is a solution of

$$
\partial_{t}^{2} u+A(t) u=G
$$

where $A(t)$ is a densely defined selfadjoint operator on $L^{2}$ with $A \in C^{1}\left([0, T] ; \mathcal{L}\left(H^{1}, H^{-1}\right)\right)$, and $G \in C^{0}\left([0, T] ; L^{2}\right)$, then

$$
\begin{aligned}
\left\|\partial_{t} u(t)\right\|_{L^{2}}^{2}+<A(t) u(t), u(t)>_{H^{-1}, H^{1}} & \\
& =\left\|\partial_{t} u(0)\right\|_{L^{2}}^{2}+<A(t) u(0), u(0)>_{H^{-1}, H^{1}}^{t} \\
& +\int_{0}^{t}<A^{\prime}(s) u(s), u(s)>_{H^{-1}, H^{1}} d s+2 \Re \int_{0}^{t}\left(G(s), \partial_{t} u(s)\right)_{L^{2}} d s .
\end{aligned}
$$


We apply this equality to $A(t):=a^{-2}(t)\left[-\Delta_{\mathbf{K}}+1\right], G:=\left(a^{-2}(t)-m^{2}-\xi R_{g}\right) u-d a^{\prime}(t) a^{-1}(t) \partial_{t} u-$ $C u|u|^{p-1}$. We note that for any $v \in C^{1}\left(I ; H^{1}(\mathbf{K})\right)$ we have

$$
\|v(t)\|_{L^{p+1}}^{p+1}-\left\|v\left(t_{0}\right)\right\|_{L^{p+1}}^{p+1}=(p+1) \Re \int_{t_{0}}^{t} v(s)|v(s)|^{p-1} \overline{\partial_{t} v(s)} d s .
$$

By density, this equality holds also for $v \in C^{0}\left(I, H^{1}\right) \cap C^{1}\left(I ; L^{2}\right)$. We deduce that a local solution $u \in C^{0}\left(\left(t_{-}^{\prime}, t_{+}^{\prime}\right), H^{1}\right) \cap C^{1}\left(\left(t_{-}^{\prime}, t_{+}^{\prime}\right) ; L^{2}\right),\left[t_{-}^{\prime}, t_{+}^{\prime}\right] \subset I$, satisfies

$$
\begin{aligned}
\left\|\partial_{t} u(t)\right\|_{L^{2}}^{2}+\frac{1}{a^{2}(t)}\|u(t)\|_{H^{1}}^{2}+\frac{2 C}{p+1} \| & u(t) \|_{L^{p+1}}^{p+1} \\
& \leqslant\left\|\partial_{t} u\left(t_{0}\right)\right\|_{L^{2}}^{2}+\frac{1}{a^{2}\left(t_{0}\right)}\left\|u\left(t_{0}\right)\right\|_{H^{1}}^{2}+\frac{2 C}{p+1}\left\|u\left(t_{0}\right)\right\|_{L^{p+1}}^{p+1} \\
& +\left|\int_{t_{0}}^{t} h(s)\left[\left\|\partial_{t} u(s)\right\|_{L^{2}}^{2}+\frac{1}{a^{2}(s)}\|u(s)\|_{H^{1}}^{2}\right] d s\right|,
\end{aligned}
$$

where $h \in C^{0}(I)$ only depends on $a, a^{\prime}, R_{g}, d, m, \xi$. The Gronwall lemma implies that there exists $k \in C^{0}\left(I^{2} \times \mathbb{R}^{2}\right)$ such that

$$
\|u(t)\|_{H^{1}}+\left\|\partial_{t} u(t)\right\|_{L^{2}} \leqslant k\left(t, t_{0},\left\|u\left(t_{0}\right)\right\|_{H^{1}},\left\|\partial_{t} u\left(t_{0}\right)\right\|_{L^{2}}\right) .
$$

Therefore $u \in L^{\infty}\left(\left(t_{-}^{\prime}, t_{+}^{\prime}\right) ; H^{1}\right), \partial_{t} u \in L^{\infty}\left(\left(t_{-}^{\prime}, t_{+}^{\prime}\right) ; L^{2}\right)$ and the integral equation $\left(u(t), \partial_{t} u(t)\right)=$ $\mathcal{F}\left(u, \partial_{t} u\right)(t)$ assures that $\left.u \in C^{0}\left(\left[t_{-}^{\prime}, t_{+}^{\prime}\right] ; H^{1}\right) \cap C^{1}\left(\left[t_{-}^{\prime}, t_{+}^{\prime}\right)\right] ; L^{2}\right)$. We conclude by the classic argument of unique continuation that $u$ is globally defined on $I$.

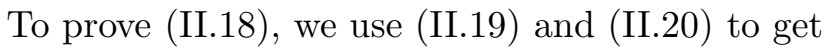

$$
\begin{aligned}
\|u(t)-\hat{u}(t)\|_{H^{1}}+\| \partial_{t} u(t)- & \partial_{t} \hat{u}(t) \|_{L^{2}} \\
\leqslant & c\left(t, t_{0}\right)\left(\left\|u\left(t_{0}\right)-\hat{u}\left(t_{0}\right)\right\|_{H^{1}}+\left\|\partial_{t} u\left(t_{0}\right)-\partial_{t} \hat{u}\left(t_{0}\right)\right\|_{L^{2}}\right) \\
& +\left|\int_{t_{0}}^{t} c(t, s)\|u(s)-\hat{u}(s)\|_{H^{1}}\left(\|u(s)\|_{H^{1}}^{p-1}+\|\hat{u}(s)\|_{H^{1}}^{p-1}\right) d s\right|
\end{aligned}
$$

for some $c \in C^{0}\left(I^{2}\right)$. Now (II.18) is a consequence of (ㅍ․22) and of Gronwall's lemma again.

Q.E.D.

These results of existence and uniqueness of the solutions being acquired, the fundamental problems lie in finding uniform energy estimates on $\left(t_{-}, t_{+}\right)$, and in investigating the asymptotic behaviours at $t_{ \pm}$. To overcome the difficulties linked to the variable speed due to the term $a^{-2}(t) \Delta_{\mathbf{K}}$, it is convenient to replace the cosmic time $t$ by the conformal time $\tau$ defined by (I.5). In this time coordinate, the metric (I.1) is expressed with the scale factor $\alpha$ given by (I.9), as

$$
g_{\mu \nu} d x^{\mu} d x^{\nu}=\alpha^{2}(\tau)\left[d \tau^{2}-\gamma_{i j} d \mathbf{x}^{i} d \mathbf{x}^{j}\right], \mathcal{M}=\left(\tau_{-}, \tau_{+}\right)_{\tau} \times \mathbf{K}_{\mathbf{x}}
$$

and the Ricci scalar is given by

$$
R_{g}=\frac{1}{\alpha^{2}(\tau)} R_{\gamma}+2 d \frac{\alpha^{\prime \prime}(\tau)}{\alpha^{3}(\tau)}+d(d-3) \frac{\alpha^{\prime 2}(\tau)}{\alpha^{4}(\tau)} .
$$

Hence $u$ is a solution of (II.1) iff its Liouville transform $\varphi$ defined by (I.6) is a solution of (II.24)

$$
\left\{\partial_{\tau}^{2}-\Delta_{\mathbf{K}}+m^{2} \alpha^{2}(\tau)+\xi R_{\gamma}+d\left(\xi-\frac{d-1}{4 d}\right)\left((d-3) \frac{\alpha^{\prime 2}(\tau)}{\alpha^{2}(\tau)}+2 \frac{\alpha^{\prime \prime}(\tau)}{\alpha(\tau)}\right)\right\} \varphi=[\alpha(\tau)]^{\frac{d+3}{2}} F
$$

and the initial data (II.2) on $u$ are transformed by an isomorphism $\mathcal{L}$ on $H^{s} \times H^{s-1}$,

$$
\mathcal{L}:\left(u_{0}, u_{1}\right) \mapsto\left(\varphi_{0}, \varphi_{1}\right), \quad \varphi_{0}=\left[a\left(t_{0}\right)\right]^{\frac{d-1}{2}} u_{0}, \quad \varphi_{1}=\frac{d-1}{2} a^{\prime}\left(t_{0}\right)\left[a\left(t_{0}\right)\right]^{\frac{d-1}{2}} u_{0}+\left[a\left(t_{0}\right)\right]^{\frac{d+1}{2}} u_{1} .
$$


In the case of the conformal coupling $\xi=\frac{d-1}{4 d}$, this equation takes a simpler and more tractable form. The linear equation (I.4) is equivalent to

$$
\left\{\partial_{\tau}^{2}-\Delta_{\mathbf{K}}+m^{2} \alpha^{2}(\tau)+\frac{d-1}{4 d} R_{\gamma}\right\} \varphi=0,
$$

and the semilinear equation (I.19) with (I.20), becomes

$$
\begin{gathered}
\left\{\partial_{\tau}^{2}-\Delta_{\mathbf{K}}+m^{2} \alpha^{2}(\tau)+\frac{d-1}{4 d} R_{\gamma}\right\} \varphi=-C[\alpha(\tau)]^{\nu} \varphi|\varphi|^{p-1}, \\
\nu:=\frac{d+3-p(d-1)}{2} \in\left[\frac{d-3}{d-2}, 2\right] .
\end{gathered}
$$

Thus for the conformal coupling, we deal with a Klein-Gordon equation with variable mass $m \alpha(\tau)$ that, as $\tau \rightarrow \tau_{ \pm}$, tends to zero for a Big Bang/Crunch, to the infinity for a Big Rip, or a constant (and $\alpha^{\prime}$ is diverging) for a Sudden Singularity. Therefore the asymptotic properties of $u$ heavily depend on the behaviour of the scale factor $a(t)$ near the singularities $t_{ \pm}$. In the Appendix we present the asymptotics of $\alpha(\tau)$ near $\tau_{ \pm}$.

\section{At a Big Bang/Crunch or a Sudden Singularity (Conformal Coupling)}

In this part we investigate the asymptotics at a Big Bang/Crunch or a Sudden Singularity, of a massive scalar field with the conformal coupling (I.16), solving the linear or nonlinear Klein-Gordon equation (II.1) with $F$ given by (II.17). We consider only the case of a future time singularity at $t_{+}$, the case at $t_{-}$is easily deduced by reversing time. First we remark that for $0 \leqslant \eta_{+}^{0}<1$, we have $\tau_{+}<\infty$ and $\alpha \in C^{0}\left(\left[0, \tau_{+}\right]\right)$. In this simple situation, it is sufficient to prove that the solution of (II.26) is well defined at $\tau_{+}$and the Cauchy problem is well posed for initial data specified at $\tau_{+}$. In contrast, for $\eta_{0}^{+} \geqslant 1$, we have $\tau_{+}=+\infty$ and since $\alpha(\tau) \rightarrow 0$ as $\tau \rightarrow \infty$, we compare the solutions of (II.26) near $\tau_{+}$with those of the massless equation

$$
\left\{\partial_{\tau}^{2}-\Delta_{\mathbf{K}}+\frac{d-1}{4 d} R_{\gamma}\right\} \varphi=0 .
$$

Therefore we deal with a scattering problem. The Cauchy problem is well posed for this equation: given $\varphi_{0} \in H^{1-\theta}(\mathbf{K}), \varphi_{1} \in H^{-\theta}(\mathbf{K})$ with $\theta \in[0,1]$, there exists a $\varphi \in C^{0}\left(\left(\tau_{-}, \tau_{+}\right) ; H^{1-\theta}(\mathbf{K})\right) \cap$ $C^{1}\left(\left(\tau_{-}, \tau_{+}\right) ; H^{-\theta}(\mathbf{K})\right)$ solution of (III.1) satisfying

$$
\varphi(0)=\varphi_{0}, \partial_{\tau} \varphi(0)=\varphi_{1} .
$$

We remark that in some cases, the solutions of (III.1) can be unbounded as $\tau \rightarrow \infty$ : the Kato Rellich theorem and (II.3) assure that $-\Delta_{\mathbf{K}}+\frac{d-1}{4 d} R_{\gamma}$ endowed with the domain $H^{2}(\mathbf{K})$ is selfadjoint on $L^{2}$, hence if its spectrum intersects $(-\infty, 0]$ the norm of some solutions can increase as $\tau \rightarrow+\infty$. To avoid this situation we introduce an "infrared" cut-off. Given $\delta \geqslant 0$, we introduce

$$
H^{2, \delta}(\mathbf{K}):=\left\{\varphi \in H^{2}(\mathbf{K}) ; \chi_{(-\infty, \delta)}\left(-\Delta_{\mathbf{K}}+\frac{d-1}{4 d} R_{\gamma}\right) \varphi=0\right\}
$$

where $\chi_{\Omega}$ is the characteristic function of $\Omega \subset \mathbb{R}$, and for $s \leqslant 2$ we define $H^{s, \delta}(\mathbf{K})$ as the closure of $H^{2, \delta}(\mathbf{K})$ for the norm (II.4).

First, we investigate the asymptotic behaviours of the free fields solving the linear Klein-Gordon equation.

Theorem III.1 (Big Crunch, Sudden Singularity). We assume that (I.2), (I.3) and (II.3) and hold. Given $\theta \in[0,1], u_{0} \in H^{1-\theta}(\mathbf{K}), u_{1} \in H^{-\theta}(\mathbf{K})$, we consider the unique $u \in C^{0}\left(I ; H^{1-\theta}(\mathbf{K})\right) \cap$ 
$C^{1}\left(I ; H^{-\theta}(\mathbf{K})\right)$ solution of $(I .4)$, (I.16), (II.2).

If $0 \leqslant \eta_{0}^{+}<1$, there exist unique $\varphi_{0}^{+} \in H^{1-\theta}(\mathbf{K}), \varphi_{1}^{+} \in H^{-\theta}(\mathbf{K})$, such that

$$
\left\|[a(t)]^{\frac{d-1}{2}} u(t, .)-\varphi_{0}^{+}\right\|_{H^{1-\theta}}+\left\|a(t) \partial_{t}\left[[a(t)]^{\frac{d-1}{2}} u(t, .)\right]-\varphi_{1}^{+}\right\|_{H^{-\theta}} \longrightarrow 0, t \rightarrow t_{+},
$$

and the map $W_{+}:\left(u_{0}, u_{1}\right) \mapsto\left(\varphi_{0}^{+}, \varphi_{1}^{+}\right)$is an isomorphism on $H^{1-\theta}(\mathbf{K}) \times H^{-\theta}(\mathbf{K})$. For any $\delta \in \mathbb{R}$, $H^{1-\theta, \delta}(\mathbf{K}) \times H^{-\theta, \delta}(\mathbf{K})$ is invariant under $W_{+}$.

If $1 \leqslant \eta_{0}^{+}$, given $0<\delta, u_{0} \in H^{1-\theta, \delta}(\mathbf{K}), u_{1} \in H^{-\theta, \delta}(\mathbf{K})$, there exist unique $\varphi_{0}^{+} \in H^{1-\theta, \delta}(\mathbf{K})$, $\varphi_{1}^{+} \in H^{-\theta, \delta}(\mathbf{K})$, such that

$$
\left\|[a(t)]^{\frac{d-1}{2}} u(t, .)-\varphi(\tau)\right\|_{H^{1-\theta}}+\left\|a(t) \partial_{t}\left[[a(t)]^{\frac{d-1}{2}} u(t, .)\right]-\partial_{\tau} \varphi(\tau)\right\|_{H^{-\theta}} \longrightarrow 0, \quad t \rightarrow t_{+},
$$

where $\varphi$ is the solution of (III.1) satisfying $\varphi(0)=\varphi_{0}^{+}, \partial_{\tau} \varphi(0)=\varphi_{1}^{+}$, and $\tau$ is defined by (I.5). The map $W_{+}:\left(u_{0}, u_{1}\right) \mapsto\left(\varphi_{0}^{+}, \varphi_{1}^{+}\right)$is an isomorphism on $H^{1-\theta, \delta}(\mathbf{K}) \times H^{-\theta, \delta}(\mathbf{K})$.

If $0 \leqslant \eta_{0}^{+}, \theta=\frac{1}{2}$ and $\sigma$ is given by (II.6), we have

$$
\sigma(u, \tilde{u})=\left\langle\varphi_{0}^{+}, \tilde{\varphi}_{1}^{+}\right\rangle-\left\langle\tilde{\varphi}_{0}^{+}, \varphi_{1}^{+}\right\rangle
$$

where $\left(\tilde{\varphi}_{0}^{+}, \tilde{\varphi}_{1}^{+}\right)=W_{+}\left(\tilde{u}\left(t_{0}\right), \partial_{t} \tilde{u}\left(t_{0}\right)\right)$.

Proof. We introduce the group $U_{0}(\tau)$ associated to equation (III.1) and defined by $U_{0}(\tau)\left(\varphi_{0}, \varphi_{1}\right)=$ $\left(\varphi(\tau), \partial_{\tau} \varphi(\tau)\right)$ if $\varphi \in C^{0}\left(\mathbb{R}_{\tau} ; H^{1-\theta}(\mathbf{K})\right) \cap C^{1}\left(\mathbb{R}_{\tau} ; H^{-\theta}(\mathbf{K})\right)$ is the solution of (III.1) with initial data $\varphi(0)=\varphi_{0}, \partial_{\tau} \varphi(0)=\varphi_{1}$. For any $a, b \in \mathbb{R}, a<b$, there exists $C_{b-a}>0$ such that

$$
\sup _{\tau \in[a, b]}\left\|U_{0}(\tau)\right\|_{\mathcal{L}\left(H^{1-\theta} \times H^{-\theta}\right)} \leqslant C_{b-a}<\infty .
$$

Given $u_{0} \in H^{1-\theta}(\mathbf{K}), u_{1} \in H^{-\theta}(\mathbf{K})$, we choose $\left(\varphi_{0}, \varphi_{1}\right)=\mathcal{L}_{t_{0}}\left(u_{0}, u_{1}\right)$ given by (II.25). Now the solution of (II.26) with initial data $\left(\varphi_{0}, \varphi_{1}\right)$ satisfies for $\tau \in\left(\tau_{-}, \tau_{+}\right)$

$$
\left(\varphi(\tau), \partial_{\tau} \varphi(\tau)\right)=U_{0}(\tau)\left(\varphi_{0}, \varphi_{1}\right)-m^{2} \int_{0}^{\tau} U_{0}(\tau-\sigma)\left(0, \alpha^{2}(\sigma) \varphi(\sigma)\right) d \sigma
$$

Then the Gronwall lemma implies that for $\tau \in\left[0, \tau_{+}\right)$

$$
\|\varphi(\tau)\|_{H^{1-\theta}}+\left\|\partial_{\tau} \varphi(\tau)\right\|_{H^{-\theta}} \leqslant C_{\tau_{+}}\left(\left\|\varphi_{0}\right\|_{H^{1-\theta}}+\left\|\varphi_{1}\right\|_{H^{-\theta}}\right) \exp \left(C_{\tau_{+}} m^{2} \int_{0}^{\tau} \alpha^{2}(\sigma) d \sigma\right) .
$$

For $\eta_{0}^{+} \in[0,1)$ we have $\alpha \in C^{0}\left(\left[0, \tau_{+}\right]\right)$hence

$$
\left(\varphi, \partial_{\tau} \varphi\right) \in L^{\infty}\left(\left(0, \tau_{+}\right) ; H^{1-\theta} \times H^{-\theta}\right),
$$

therefore (III.8) assures that $\left(\varphi(\tau), \partial_{\tau} \varphi(\tau)\right)$ has a limit as $\tau \rightarrow \tau_{+}$, and $\varphi_{0}^{+}, \varphi_{1}^{+}$defined by

$$
\left(\varphi_{0}^{+}, \varphi_{1}^{+}\right)=U_{0}\left(\tau_{+}\right)\left(\varphi_{0}, \varphi_{1}\right)-m^{2} \int_{0}^{\tau_{+}} U_{0}\left(\tau_{+}-\sigma\right)\left(0, \alpha^{2}(\sigma) \varphi(\sigma)\right) d \sigma
$$

satisfy (III.4). Conversely, given $\left(\varphi_{0}^{+}, \varphi_{1}^{+}\right) \in H^{1-\theta} \times H^{-\theta}$, we can easily solve, by Picard iteration, the integral equation

$$
\left(\varphi(\tau), \partial_{\tau} \varphi(\tau)\right)=U_{0}\left(\tau-\tau_{+}\right)\left(\varphi_{0}^{+}, \varphi_{1}^{+}\right)-m^{2} \int_{\tau_{+}}^{\tau} U_{0}(\tau-\sigma)\left(0, \alpha^{2}(\sigma) \varphi(\sigma)\right) d \sigma
$$

for $\tau \in\left(\tau_{-}, \tau_{+}\right]$.. In other words, the Cauchy problem for (II.26) is well posed in $C^{0}\left(\left(\tau_{-}, \tau_{+}\right] ; H^{1-\theta} \times H^{-\theta}\right)$ with initial data specified at $\tau_{+}$. Now it is sufficient to define $\left(\varphi_{0}, \varphi_{1}\right)$ from (III.11) and we conclude that $\left(u_{0}, u_{1}\right)$ given by (II.25) satisfy (III.4). To obtain the invariance of $H^{1-\theta, \delta} \times H^{-\theta, \delta}$ it is 
sufficient to remark that $U_{0}(\tau)$ lets invariant this space.

If $\eta_{0}^{+} \geqslant 1$, we have $\tau_{+}=+\infty$ and $\left\|U_{0}(\tau)\right\|_{\mathcal{L}\left(H^{1-\theta} \times H^{-\theta}\right)}$ can be unbounded on $[0, \infty)$, hence we shall introduce an infrared cut-off. First we have to define equivalent norms on $H^{s}(\mathbf{K})$. We consider the operators

$$
\Lambda_{0}:=-\Delta_{\mathbf{K}}+1, \quad A_{0}:=-\Delta_{\mathbf{K}}+\frac{d-1}{4 d} R_{\gamma}, \quad \Lambda_{1}:=A_{0}+1+\frac{d-1}{4 d}\left\|R_{\gamma}\right\|_{L^{\infty}} .
$$

By the Kato-Rellich theorem $A_{0}, \Lambda_{1}$ are selfadjoint operators with the same domain as $\Lambda_{0}$ :

$$
H^{2}(\mathbf{K})=\mathcal{D}\left(A_{0}\right)=\mathcal{D}\left(\Lambda_{0}\right)=\mathcal{D}\left(\Lambda_{1}\right) .
$$

Since $1 \leqslant \Lambda_{j}$ we may define $\Lambda_{j}^{\theta}$ for $\theta \in[0,1]$ by the spectral theorem and it is well known from complex interpolation (see [39], Remark 2.3, p.10) that

$$
H^{2 \theta}(\mathbf{K})=\mathcal{D}\left(\Lambda_{0}^{\theta}\right)=\mathcal{D}\left(\Lambda_{1}^{\theta}\right) \text { with equivalent norms } .
$$

Given $\delta>0$, we introduce the truncated at low frequency operator

$$
A_{\delta}:=\chi_{[\delta, \infty)}\left(A_{0}\right) A_{0}, \mathcal{D}\left(A_{\delta}\right)=H^{2, \delta}(\mathbf{K}),
$$

which is selfadjoint on $L_{\delta}^{2}:=H^{0, \delta}(\mathbf{K})$ and $\delta I \leqslant A_{\delta}$. We remark that the norms

$$
\left\|\Lambda_{1}^{\theta} u\right\|_{L^{2}}, \quad\left\|A_{\delta}^{\theta} u\right\|_{L^{2}}
$$

are equivalent on $H^{2 \theta, \delta}(\mathbf{K}), \theta \in[0,1]$, hence by duality, the same result holds for $\theta \in[-1,0]$. We conclude with (III.13) that $\left\|A_{\delta}^{\theta} u\right\|_{L^{2}}$ is equivalent to the $H^{2 \theta}$-norm on $H^{2 \theta, \delta}$ given by $\left\|A_{0}^{\theta} u\right\|_{L^{2}}$. We remark that the group $U_{0}(\tau)$ leaves invariant $H^{1-\theta, \delta} \times H^{-\theta, \delta}$ and on this space the solution of (III.1), (III.2) can be expressed as

$$
\varphi(\tau)=\cos \left(\tau A_{\delta}^{\frac{1}{2}}\right) \varphi_{0}+A_{\delta}^{-\frac{1}{2}} \sin \left(\tau A_{\delta}^{\frac{1}{2}}\right) \varphi_{1} .
$$

As a consequence the restriction of $U_{0}(\tau)$ on $H^{1-\theta, \delta} \times H^{-\theta, \delta}$ is uniformly bounded: Given $\theta \in[0,1]$, $\delta>0$, there exists $C_{\delta}>0$ such that

$$
\sup _{\tau \in \mathbb{R}}\left\|U_{0}(\tau)\right\|_{\mathcal{L}\left(H^{1-\theta, \delta} \times H^{-\theta, \delta}\right)} \leqslant C_{\delta}<\infty .
$$

We deduce from (III.8) that the propagator associated to (II.26) leaves invariant $H^{1-\theta, \delta} \times H^{-\theta, \delta}$ and since $\alpha \in L^{1}(0, \infty)$, the Gronwall lemma assures that the solution of (II.26) with initial data in $H^{1-\theta, \delta} \times H^{-\theta, \delta}$ satisfies for $\sigma, \tau \in[0, \infty)$

(III.16)

$$
\left\|\left(\varphi(\tau), \partial_{\tau} \varphi(\tau)\right)\right\|_{H^{1-\theta, \delta} \times H^{-\theta, \delta}} \leqslant C_{\delta}\left[\left\|\left(\varphi(\sigma), \partial_{\tau} \varphi(\sigma)\right)\right\|_{H^{1-\theta, \delta} \times H^{-\theta, \delta}}+\exp \left(m^{2} C_{\delta} \int_{0}^{\infty} \alpha^{2}\left(\tau^{\prime}\right) d \tau^{\prime}\right)\right] .
$$

Therefore $U_{0}(-\tau)\left(0, \alpha^{2}(\tau) \varphi(\tau)\right)$ belongs to $L^{1}\left(0, \infty ; H^{1-\theta, \delta} \times H^{-\theta, \delta}\right)$ and the following limit exists

$$
\left(\varphi_{0}^{+}, \varphi_{1}^{+}\right):=\lim _{\tau \rightarrow \infty} U_{0}(-\tau)\left(\varphi(\tau), \partial_{\tau} \varphi(\tau)\right)=\left(\varphi_{0}, \varphi_{1}\right)-m^{2} \int_{0}^{\infty} U_{0}(-\tau)\left(0, \alpha^{2}(\tau) \varphi(\tau)\right) d \tau
$$

and satisfies (III.5). Conversely, given $\left(\varphi_{0}^{+}, \varphi_{1}^{+}\right) \in H^{1-\theta, \delta} \times H^{-\theta, \delta}$, we construct $\left(\varphi_{0}, \varphi_{1}\right)$ using the principle of the Cook method (see e.g. [45]): the time integrability of the perturbation. We introduce the propagator $U(\tau, \sigma)$ associated to (II.26) defined by $U(\tau, \sigma)\left(\varphi_{0}, \varphi_{1}\right)=\left(\varphi(\tau), \partial_{\tau} \varphi(\tau)\right)$ if $\varphi$ is the the solution of (II.26) with initial data at time $\sigma,\left(\varphi(\sigma), \partial_{\tau}(\sigma)\right)=\left(\varphi_{0}, \varphi_{1}\right)$. (III.16) assures that there exists $C_{\delta}^{\prime}<\infty$ such that

$$
\sup _{0 \leqslant \sigma, \tau}\|U(\tau, \sigma)\|_{\mathcal{L}\left(H^{1-\theta} \times H^{-\theta}\right)} \leqslant C_{\delta}^{\prime} .
$$


If $\left(\varphi_{0}^{+}, \varphi_{1}^{+}\right) \in H^{2, \delta} \times H^{1, \delta}$, Proposition $\coprod .2$ implies that $\sigma \mapsto U(0, \sigma) U_{0}(\sigma)\left(\varphi_{0}^{+}, \varphi_{1}^{+}\right)$belongs to $C^{1}\left([0, \infty) ; H^{1, \delta} \times H^{0, \delta}\right)$ and

$$
\frac{d}{d \sigma} U(0, \sigma) U_{0}(\sigma)\left(\varphi_{0}^{+}, \varphi_{1}^{+}\right)=m^{2} \alpha^{2}(\sigma) U(0, \sigma)\left(0, \varphi_{0}(\sigma)\right), \quad U_{0}(\sigma)\left(\varphi_{0}^{+}, \varphi_{1}^{+}\right)=\left(\varphi_{0}(\sigma), \partial_{\tau} \varphi_{0}(\sigma)\right),
$$

hence

$$
\left\|U(0, \tau) U_{0}(\tau)\left(\varphi_{0}^{+}, \varphi_{1}^{+}\right)-U(0, \sigma) U_{0}(\sigma)\left(\varphi_{0}^{+}, \varphi_{1}^{+}\right)\right\|_{H^{1-\theta} \times H^{-\theta}} \leqslant m^{2} C_{\delta} C_{\delta}^{\prime}\left|\int_{\sigma}^{\tau} \alpha^{2}\left(\tau^{\prime}\right) d \tau^{\prime}\right| .
$$

By density, this inequality holds for $\left(\varphi_{0}^{+}, \varphi_{1}^{+}\right) \in H^{1-\theta, \delta} \times H^{-\theta, \delta}$. Therefore the following limit exists in this space,

and satisfies (III.5).

$$
\left(\varphi_{0}, \varphi_{1}\right):=\lim _{\tau \rightarrow \infty} U(0, \tau) U_{0}(\tau)\left(\varphi_{0}^{+}, \varphi_{1}^{+}\right)
$$

Finally to prove (III.6) we remark that if $\tilde{\varphi}(\tau)=[a(t)]^{\frac{d-1}{2}} \tilde{u}(t)$, we have $\sigma(u, \tilde{u})=\left\langle\varphi(\tau), \partial_{\tau} \tilde{\varphi}(\tau)\right\rangle-\left\langle\tilde{\varphi}(\tau), \partial_{\tau} \varphi(\tau)\right\rangle=\lim _{\tau \rightarrow \infty}\left\langle\varphi(\tau), \partial_{\tau} \tilde{\varphi}(\tau)\right\rangle-\left\langle\tilde{\varphi}(\tau), \partial_{\tau} \varphi(\tau)\right\rangle=\left\langle\varphi_{0}^{+}, \tilde{\varphi}_{1}^{+}\right\rangle-\left\langle\tilde{\varphi}_{0}^{+}, \varphi_{1}^{+}\right\rangle$. Q.E.D.

We now investigate the non-linear problem. We recall that by the Liouville transform, the semi-linear Klein-Gordon equation (II.1), (II.17) is equivalent to (II.27).

Theorem III.2. We assume that $(\mathbf{K}, \gamma)$ is a $C^{\infty}$ bounded geometry manifold. Let a be a strictly positive function in $C^{2}(I)$ satisfying (I.2), (I.3), and $m \geqslant 0$. Given $u_{0} \in H^{1}(\mathbf{K}), u_{1} \in L^{2}(\mathbf{K})$, we consider the unique $u \in C^{0}\left(I ; H^{1}(\mathbf{K})\right) \cap C^{1}\left(I ; L^{2}(\mathbf{K})\right)$ solution of (II.1), (II.2), (II.17).

We suppose that $0 \leqslant \eta_{0}^{+}<1$, and if $\eta_{0}^{+}=0$ we assume $c_{1}^{+}>0$. Then there exist unique $\varphi_{0}^{+} \in H^{1}(\mathbf{K}), \varphi_{1}^{+} \in L^{2}(\mathbf{K})$, such that

$$
\left\|[a(t)]^{\frac{d-1}{2}} u(t, .)-\varphi_{0}^{+}\right\|_{H^{1}}+\left\|a(t) \partial_{t}\left[[a(t)]^{\frac{d-1}{2}} u(t, .)\right]-\varphi_{1}^{+}\right\|_{L^{2}} \longrightarrow 0, t \rightarrow t_{+} .
$$

If $1 \leqslant \eta_{0}^{+}$, we assume that there exists $\delta>0$ such that

$$
\delta \leqslant R_{\gamma}
$$

and the nonlinearity satisfies

$$
\left\{\begin{array}{l}
\text { if } \eta_{0}^{+}=1 \text { and } d=3,1 \leqslant p<3, \\
\text { if } \eta_{0}^{+}=1 \text { and } d \geqslant 4,1 \leqslant p \leqslant \frac{d}{d-2}, \\
\text { if } 1<\eta_{0}^{+}<d-2,1 \leqslant p \leqslant \frac{d}{d-2}, \\
\text { if } \eta_{0}^{+}=d-2,1 \leqslant p<\frac{d}{d-2}, \\
\text { if } \eta_{0}^{+}>d-2,1 \leqslant p<1+\frac{2}{d-1}\left(1+\frac{1}{\eta_{0}^{+}}\right) .
\end{array}\right.
$$

Then, given $u_{0} \in H^{1}(\mathbf{K}), u_{1} \in L^{2}(\mathbf{K})$, there exist unique $\varphi_{0}^{+} \in H^{1}(\mathbf{K}), \varphi_{1}^{+} \in L^{2}(\mathbf{K})$, such that the solution u satisfies

$$
\left\|[a(t)]^{\frac{d-1}{2}} u(t, .)-\varphi(\tau)\right\|_{H^{1}}+\left\|a(t) \partial_{t}\left[[a(t)]^{\frac{d-1}{2}} u(t, .)\right]-\partial_{\tau} \varphi(\tau)\right\|_{L^{2}} \longrightarrow 0, \quad t \rightarrow t_{+},
$$

where $\varphi$ is the solution of (III.1) with initial data $\varphi(0)=\varphi_{0}^{+}, \partial_{\tau} \varphi(0)=\varphi_{1}^{+}$.

For any $\eta_{0}^{+} \geqslant 0$, the map

$$
\Omega_{+}:\left(u_{0}, u_{1}\right) \mapsto\left(\varphi_{0}^{+}, \varphi_{1}^{+}\right)
$$

is a bicontinuous bijection on $H^{1}(\mathbf{K}) \times L^{2}(\mathbf{K})$ and there exists a continuous function $\Psi$ such that (III.23)

$\left\|\Omega_{+}\left(u_{0}, u_{1}\right)-\Omega_{+}\left(\hat{u}_{0}, \hat{u}_{1}\right)\right\|_{H^{1} \times L^{2}} \leqslant\left\|\left(u_{0}, u_{1}\right)-\left(\hat{u}_{0}, \hat{u}_{1}\right)\right\|_{H^{1} \times L^{2}} \Psi\left(\left\|\left(u_{0}, u_{1}\right)\right\|_{H^{1} \times L^{2}}+\left\|\left(\hat{u}_{0}, \hat{u}_{1}\right)\right\|_{H^{1} \times L^{2}}\right)$, 
(III.24)

$$
\left\|\Omega_{+}^{-1}\left(\varphi_{0}^{+}, \varphi_{1}^{+}\right)-\Omega_{+}^{-1}\left(\hat{\varphi}_{0}^{+}, \hat{\varphi}_{1}^{+}\right)\right\|_{H^{1} \times L^{2}} \leqslant\left\|\left(\varphi_{0}^{+}, \varphi_{1}^{+}\right)-\left(\hat{\varphi}_{0}^{+}, \hat{\varphi}_{1}^{+}\right)\right\|_{H^{1} \times L^{2}} \Psi\left(\left\|\left(\varphi_{0}^{+}, \varphi_{1}^{+}\right)\right\|_{H^{1} \times L^{2}}+\left\|\left(\hat{\varphi}_{0}^{+}, \hat{\varphi}_{1}^{+}\right)\right\|_{H^{1} \times L^{2}}\right) .
$$

Remark III.3. Obviously many issues on the asymptotics for the semi-linear Klein-Gordon equation remain open, and the assumptions (III.20) could seem to be too strong. Nevertheless we remark that the existence of a free asymptotic field fails in an important limit case. If $\mathbf{K}$ is a compact manifold with $R_{\gamma}=6, d=3, \eta_{0}^{+}=1, C=1, p=3, \xi=1 / 6$, the semilinear Klein-Gordon equation satisfied by a field constant on $\mathbf{K}$ is the quartic oscillator. Then given $\varphi_{0} \in \mathbb{R}^{*}, \varphi_{1}=0$, the solution $\varphi(\tau, \mathbf{x})=\varphi(\tau)$ of (II.27) with $\varphi(0)=\varphi_{0}, \varphi^{\prime}(0)=0$, satisfies the Duffin equation $\varphi^{\prime \prime}+\varphi+\varphi^{3}=0$ and $\varphi$ is expressed with the Jacobi function $\operatorname{cn}(z, k)$ (see [41], p. 565):

$$
\varphi(\tau)=\varphi_{0} \mathrm{cn}\left(\tau \sqrt{1+\frac{\varphi_{0}^{2}}{2}}, \frac{1}{\sqrt{2+\frac{2}{\varphi_{0}^{2}}}}\right) .
$$

Hence $\varphi$ is periodic with the period

$$
T\left(\varphi_{0}\right)=\frac{4}{\sqrt{1+\frac{\varphi_{0}^{2}}{2}}} \int_{0}^{\pi / 2} \frac{1}{\sqrt{1-\frac{\varphi_{0}^{2}}{2\left(\varphi_{0}^{2}+1\right)} \sin ^{2} \theta}} d \theta
$$

Using inequality (19.9.1) of [41], we can estimate this period for $\varphi_{0} \neq 0$,

$$
T\left(\varphi_{0}\right) \leqslant \frac{2 \pi}{\sqrt{1+\frac{\varphi_{0}^{2}}{2}}}\left(1+\frac{1}{\pi} \ln \left(1+\frac{\varphi_{0}^{2}}{\varphi_{0}^{2}+2}\right)\right)<2 \pi,
$$

therefore we conclude that $\varphi$ can not be asymptotic to a free field solution of (III.1) which, in our case, is reduced to the simple harmonic oscillator $\varphi^{\prime \prime}+\varphi=0$.

Proof. If $0 \leqslant \eta_{0}^{+}<1$, the existence of the isomorphism $\Omega_{+}$is assured iff the global Cauchy problem associated to (II.27) with initial data given at time $\tau_{0}$ fixed in $\left[0, \tau_{+}\right]$is well posed in $C^{0}\left(\left[0, \tau_{+}\right] ; H^{1}(\mathbf{K})\right) \cap C^{1}\left(\left[0, \tau_{+}\right] ; L^{2}(\mathbf{K})\right)$. Since Theorem 11.3 assures that the global Cauchy problem is well posed on $\left[0, \tau_{+}\right)$, it is sufficient to study it on $\left[\tau_{0}^{\prime}, \tau_{+}\right]$for some $\tau_{0}^{\prime}<\tau_{+}$. We choose $\tau_{0}^{\prime}$ such that $\alpha^{\prime}(\tau) \leqslant 0$ on $\left[\tau_{0}^{\prime}, \tau_{+}\right)$. Such a choice is possible since $c_{0}^{+}>0$ and, if $\eta_{0}^{+}=0, c_{1}>0$. In the proof of the previous theorem, we have proved that the Cauchy problem for the linear equation (II.26) is solved by a propagator

$$
U\left(\tau, \tau_{0}\right) \in C^{0}\left(\left[0, \tau_{+}\right] \times\left[0, \tau_{+}\right] ; \mathcal{L}\left(H^{1} \times L^{2}\right)\right) .
$$

Therefore given $\varphi_{0} \in H^{1}, \varphi_{1} \in L^{2}, \tau_{0} \in\left[\tau_{0}^{\prime}, \tau_{+}\right]$, we have to solve the integral equation

$$
\left(\varphi(\tau), \partial_{\tau} \varphi(\tau)\right)=U\left(\tau, \tau_{0}\right)\left(\varphi_{0}, \varphi_{1}\right)+\int_{\tau_{0}}^{\tau} U(\tau, \sigma)\left(0,-C[\alpha(\sigma)]^{\nu} \varphi(\sigma)|\varphi(\sigma)|^{p-1}\right) d \sigma
$$

Using the Lipschitz property (II.19), it is easy to get a unique local solution defined near $\tau_{0}$ for $\left|\tau-\tau_{0}\right| \leqslant \epsilon$ small enough. To obtain the global existence, we apply (II.21) with $A(\tau)=-\Delta_{\mathbf{K}}+$ 
$1+m^{2} \alpha^{2}(\tau)$ and $G=\varphi-\frac{d-1}{4 d} R_{\gamma} \varphi-C[\alpha(\tau)]^{\nu} \varphi|\varphi|^{p-1}$. We get for any $\tau, \tau_{0}$

$$
\begin{aligned}
\left\|\partial_{\tau} \varphi(\tau)\right\|_{L^{2}}^{2} & +\|\varphi(\tau)\|_{H^{1}}^{2}+m^{2} \alpha^{2}(\tau)\|\varphi(\tau)\|_{L^{2}}^{2}+\frac{2 C}{p+1}[\alpha(\tau)]^{\nu}\|\varphi(\tau)\|_{L^{p+1}}^{p+1} \\
& =\left\|\partial_{\tau} \varphi\left(\tau_{0}\right)\right\|_{L^{2}}^{2}+\left\|\varphi\left(\tau_{0}\right)\right\|_{H^{1}}^{2}+m^{2} \alpha^{2}\left(\tau_{0}\right)\left\|\varphi\left(\tau_{0}\right)\right\|_{L^{2}}^{2}+\frac{2 C}{p+1}\left[\alpha\left(\tau_{0}\right)\right]^{\nu}\left\|\varphi\left(\tau_{0}\right)\right\|_{L^{p+1}}^{p+1} \\
& +2 \int_{\tau_{0}}^{\tau} \alpha^{\prime}(\sigma)\left[m^{2} \alpha(\sigma)\|\varphi(\sigma)\|_{L^{2}}^{2}+\frac{C \nu}{p+1}\left[\alpha(\sigma]^{\nu-1}\|\varphi(\sigma)\|_{L^{p+1}}^{p+1}\right] d \sigma\right. \\
& +2 \Re \int_{\tau_{0}}^{\tau}\left\langle\left(1-\frac{d-1}{4 d} R_{\gamma}\right) \varphi(\sigma), \partial_{\tau} \varphi(\sigma)\right\rangle_{L^{2}} d \sigma
\end{aligned}
$$

Since $\alpha^{\prime} \leqslant 0$ on $\left[\tau_{0}^{\prime}, \tau_{+}\right)$we deduce that

$$
\begin{aligned}
\left\|\partial_{\tau} \varphi(\tau)\right\|_{L^{2}}^{2} & +\|\varphi(\tau)\|_{H^{1}}^{2}+m^{2} \alpha^{2}(\tau)\|\varphi(\tau)\|_{L^{2}}^{2}+\frac{2 C}{p+1}[\alpha(\tau)]^{\nu}\|\varphi(\tau)\|_{L^{p+1}}^{p+1} \\
& \leqslant\left\|\partial_{\tau} \varphi\left(\tau_{0}\right)\right\|_{L^{2}}^{2}+\left\|\varphi\left(\tau_{0}\right)\right\|_{H^{1}}^{2}+m^{2} \alpha^{2}\left(\tau_{0}\right)\left\|\varphi\left(\tau_{0}\right)\right\|_{L^{2}}^{2}+\frac{2 C}{p+1}\left[\alpha\left(\tau_{0}\right)\right]^{\nu}\left\|\varphi\left(\tau_{0}\right)\right\|_{L^{p+1}}^{p+1} \\
& +\int_{\tau_{0}}^{\tau}\left(1+\left\|R_{\gamma}\right\|_{L^{\infty}}\right)\left(\left\|\partial_{\tau} \varphi(\sigma)\right\|_{L^{2}}^{2}+\|\varphi(\sigma)\|_{H^{1}}^{2}\right) d \sigma .
\end{aligned}
$$

We conclude with the Gronwall lemma that $\|\varphi(\tau)\|_{H^{1}}+\left\|\partial_{\tau} \varphi(\tau)\right\|_{L^{2}}$ is bounded and so by the continuation principle, $\varphi$ is a solution well defined on $\left[\tau_{0}^{\prime}, \tau_{+}\right]$. We have also proved that there exists a continuous function $H$ such that for any solution $\varphi$ we have

$$
\forall \tau, \tau_{0} \in\left[0, \tau_{+}\right],\|\varphi(\tau)\|_{H^{1}}+\left\|\partial_{\tau} \varphi(\tau)\right\|_{L^{2}} \leqslant H\left(\left\|\varphi\left(\tau_{0}\right)\right\|_{H^{1}}+\left\|\partial_{\tau} \varphi\left(\tau_{0}\right)\right\|_{L^{2}}\right) .
$$

Given two solutions, we write

$$
\begin{aligned}
\left(\varphi(\tau), \partial_{\tau} \varphi(\tau)\right)-\left(\hat{\varphi}(\tau), \partial_{\tau} \hat{\varphi}(\tau)\right)= & U\left(\tau, \tau_{0}\right)\left[\left(\varphi\left(\tau_{0}\right), \partial_{\tau} \varphi\left(\tau_{0}\right)\right)-\left(\hat{\varphi}(\tau), \partial_{\tau} \hat{\varphi}\left(\tau_{0}\right)\right)\right] \\
& -C \int_{\tau_{0}}^{\tau}[\alpha(\sigma)]^{\nu} U(\tau, \sigma)\left(0, \varphi(\sigma)|\varphi(\sigma)|^{p-1}-\hat{\varphi}(\sigma)|\hat{\varphi}(\sigma)|^{p-1}\right) d \sigma,
\end{aligned}
$$

hence by using the Lipschitz estimate (II.19) we get

$$
\begin{aligned}
\left\|\left(\varphi(\tau), \partial_{\tau} \varphi(\tau)\right)-\left(\hat{\varphi}(\tau), \partial_{\tau} \hat{\varphi}(\tau)\right)\right\|_{H^{1} \times L^{2}} & \lesssim\left\|\left(\varphi\left(\tau_{0}\right), \partial_{\tau} \varphi\left(\tau_{0}\right)\right)-\left(\hat{\varphi}(\tau), \partial_{\tau} \hat{\varphi}\left(\tau_{0}\right)\right)\right\|_{H^{1} \times L^{2}} \\
& +\int_{\tau_{0}}^{\tau}\|\varphi(\sigma)-\hat{\varphi}(\sigma)\|_{H^{1}}\left(\|\varphi(\sigma)\|_{H^{1}}^{p-1}+\hat{\varphi}(\sigma) \|_{H^{1}}^{p-1}\right) d \sigma .
\end{aligned}
$$

We use (III.27) and the Gronwall Lemma to obtain (III.23) and

We now consider the case $\eta_{0}^{+} \geqslant 1$. Theorem $\llbracket .3$ assures that given $u_{0} \in H^{1}, u_{1} \in L^{2}$ the nonlinear equation (II.27) has a unique solution $\varphi \in C^{0}\left([0, \infty) ; H^{1}\right) \cap C^{1}\left([0, \infty) ; L^{2}\right)$ with initial data $\left(\varphi_{0}, \varphi_{1}\right)=\mathcal{L}\left(u_{0}, u_{1}\right)$ given by (II.25). To establish the existence of $\Omega_{+}$it is sufficient to prove the existence of the nonlinear operator $\mathfrak{W}_{+}$defined by

$$
\mathfrak{W}_{+}:\left(\varphi_{0}, \varphi_{1}\right) \mapsto\left(\psi_{0}^{+}, \psi_{1}^{+}\right):=\lim _{\tau \rightarrow \infty} U(0, \tau)\left(\varphi(\tau), \partial_{\tau} \varphi(\tau)\right) \text { in } H^{1} \times L^{2},
$$

then, using operator $W_{+}$given by the previous Theorem, we put

$$
\Omega_{+}:=W_{+} \circ \mathcal{L}^{-1} \circ \mathfrak{W}_{+} \circ \mathcal{L} \text {. }
$$

We have

$$
U(0, \tau)\left(\varphi(\tau), \partial_{\tau} \varphi(\tau)\right)=\left(\varphi_{0}, \varphi_{1}\right)-C \int_{0}^{\tau}[\alpha(\sigma)]^{\nu} U(0, \sigma)\left(0, \varphi(\sigma)|\varphi(\sigma)|^{p-1}\right) d \sigma
$$


From (III.17) and (III.19) we have

$$
\sup _{0 \leqslant \sigma, \tau<\infty}\|U(\tau, \sigma)\|_{\mathcal{L}\left(H^{1} \times L^{2}\right)}<\infty .
$$

Now we apply (II.21) with $A(\tau)=-\Delta_{\mathbf{K}}+m^{2} \alpha^{2}(\tau)+\frac{d-1}{4 d} R_{\gamma}$ and $G=-C[\alpha(\tau)]^{\nu} \varphi|\varphi|^{p-1}$. We get for any $\tau, \tau_{0} \geqslant 0$,

$$
\begin{aligned}
\left\|\partial_{\tau} \varphi(\tau)\right\|_{L^{2}}^{2} & +\left\|\nabla_{\mathbf{K}} \varphi(\tau)\right\|_{L^{2}}^{2}+m^{2} \alpha^{2}(\tau)\|\varphi(\tau)\|_{L^{2}}^{2}+\frac{d-1}{4 d}\left\|R_{\gamma} \varphi(\tau)\right\|_{L^{2}}^{2}+\frac{2 C}{p+1}[\alpha(\tau)]^{\nu}\|\varphi(\tau)\|_{L^{p+1}}^{p+1} \\
& =\left\|\partial_{\tau} \varphi\left(\tau_{0}\right)\right\|_{L^{2}}^{2}+\left\|\nabla_{\mathbf{K}} \varphi\left(\tau_{0}\right)\right\|_{L^{2}}^{2}+m^{2} \alpha^{2}\left(\tau_{0}\right)\left\|\varphi\left(\tau_{0}\right)\right\|_{L^{2}}^{2}+\frac{d-1}{4 d}\left\|R_{\gamma} \varphi\left(\tau_{0}\right)\right\|_{L^{2}}^{2} \\
& +\frac{2 C}{p+1}\left[\alpha\left(\tau_{0}\right)\right]^{\nu}\left\|\varphi\left(\tau_{0}\right)\right\|_{L^{p+1}}^{p+1}+2 \int_{\tau_{0}}^{\tau} \alpha^{\prime}(\sigma)\left[m^{2} \alpha(\sigma)\|\varphi(\sigma)\|_{L^{2}}^{2}+\frac{C \nu}{p+1}\left[\alpha(\sigma]^{\nu-1}\|\varphi(\sigma)\|_{L^{p+1}}^{p+1}\right] d \sigma\right.
\end{aligned}
$$

Thanks to (III.19) there exists $C_{\delta}>0$ such that for any $\tau, \tau_{0} \geqslant 0$,

$$
\begin{aligned}
\left\|\partial_{\tau} \varphi(\tau)\right\|_{L^{2}}^{2} & +C_{\delta}^{-1}\|\varphi(\tau)\|_{H^{1}}^{2}+\frac{2 C}{p+1}[\alpha(\tau)]^{\nu}\|\varphi(\tau)\|_{L^{p+1}}^{p+1} \\
& \leqslant\left\|\partial_{\tau} \varphi\left(\tau_{0}\right)\right\|_{L^{2}}^{2}+C_{\delta}\left\|\varphi\left(\tau_{0}\right)\right\|_{H^{1}}^{2}+\frac{2 C}{p+1}\left[\alpha\left(\tau_{0}\right)\right]^{\nu}\left\|\varphi\left(\tau_{0}\right)\right\|_{L^{p+1}}^{p+1} \\
& +2 \int_{\tau_{0}}^{\tau} \alpha^{\prime}(\sigma)\left[m^{2} \alpha(\sigma)\|\varphi(\sigma)\|_{L^{2}}^{2}+\frac{C \nu}{p+1}\left[\alpha(\sigma]^{\nu-1}\|\varphi(\sigma)\|_{L^{p+1}}^{p+1}\right] d \sigma .\right.
\end{aligned}
$$

Since $\alpha>0$ on $[0, \infty)$ and $\alpha^{\prime} / \alpha \in L_{l o c}^{1}([0, \infty))$, the Gronwall lemma assures the existence of a continuous function $C_{0}$ such that for any $\tau, \tau_{0} \in[0, T]$ we have

$$
\|\varphi(\tau)\|_{H^{1}}+\left\|\partial_{\tau} \varphi(\tau)\right\|_{L^{2}} \leqslant C_{0}(T)\left(\left\|\varphi\left(\tau_{0}\right)\right\|_{H^{1}}+\left\|\partial_{\tau} \varphi\left(\tau_{0}\right)\right\|_{L^{2}}+\left\|\varphi\left(\tau_{0}\right)\right\|_{L^{p+1}}^{\frac{p+1}{2}}\right) .
$$

As previously, we choose $\tau_{0}^{\prime}$ such that $\alpha^{\prime}(\tau)<0$ for $\tau \geqslant \tau_{0}^{\prime}$. We take $\tau_{0}=\tau_{0}^{\prime}$ in (III.32) so we have for $\tau \geqslant \tau_{0}^{\prime}$

$$
\begin{aligned}
\left\|\partial_{\tau} \varphi(\tau)\right\|_{L^{2}}^{2} & +C_{\delta}^{-1}\|\varphi(\tau)\|_{H^{1}}^{2}+\frac{2 C}{p+1}[\alpha(\tau)]^{\nu}\|\varphi(\tau)\|_{L^{p+1}}^{p+1} \\
& \leqslant\left\|\partial_{\tau} \varphi\left(\tau_{0}^{\prime}\right)\right\|_{L^{2}}^{2}+C_{\delta}\left\|\varphi\left(\tau_{0}^{\prime}\right)\right\|_{H^{1}}^{2}+\frac{2 C}{p+1}\left[\alpha\left(\tau_{0}^{\prime}\right)\right]^{\nu}\left\|\varphi\left(\tau_{0}^{\prime}\right)\right\|_{L^{p+1}}^{p+1} .
\end{aligned}
$$

We deduce from (III.33), (III.34) and the Sobolev estimate, that there exists a continuous function $H$ such that

$$
\begin{gathered}
\sup _{\tau \geqslant 0}\left(\left\|\partial_{\tau} \varphi(\tau)\right\|_{L^{2}}+\|\varphi(\tau)\|_{H^{1}}\right) \leqslant H\left(\left\|\partial_{\tau} \varphi(0)\right\|_{L^{2}}+\|\varphi(0)\|_{H^{1}}\right), \\
\sup _{\tau \geqslant 0}\left\|\varphi(\tau)|\varphi(\tau)|^{p-1}\right\|_{L^{2}} \leqslant H\left(\left\|\partial_{\tau} \varphi(0)\right\|_{L^{2}}+\|\varphi(0)\|_{H^{1}}\right) .
\end{gathered}
$$

On the other hand (II.28), (III.20), (IX.3) and (IX.4) imply that

$$
[\alpha(\tau)]^{\nu} \in L^{1}(0, \infty) .
$$

Therefore thanks to (III.30) and (III.36), we may define $\left(\psi_{0}^{+}, \psi_{1}^{+}\right)$as

$$
\left(\psi_{0}^{+}, \psi_{1}^{+}\right):=\left(\varphi_{0}, \varphi_{1}\right)-C \int_{0}^{\infty}[\alpha(\sigma)]^{\nu} U(0, \sigma)\left(0, \varphi(\sigma)|\varphi(\sigma)|^{p-1}\right) d \sigma .
$$


To invert $\Omega_{+}$, given $\left(\varphi_{0}^{+}, \varphi_{-}^{+}\right) \in H^{1} \times L^{2}$, it is sufficient to solve in $C^{0}\left([0, \infty) ; H^{1} \times L^{2}\right)$ the integral equation

$$
\left(\varphi(\tau), \partial_{\tau} \varphi(\tau)\right)=U(\tau, 0)\left(\psi_{0}^{+}, \psi_{1}^{+}\right)+C \int_{\tau}^{\infty}[\alpha(\sigma)]^{\nu} U(\tau, \sigma)\left(0, \varphi(\sigma)|\varphi(\sigma)|^{p-1}\right) d \sigma
$$

with

$$
\left(\psi_{0}^{+}, \psi_{1}^{+}\right)=\mathcal{L} \circ\left(W_{+}\right)^{-1}\left(\varphi_{0}^{+}, \varphi_{1}^{+}\right)
$$

and we put

$$
W_{+}^{-1}\left(\varphi_{0}^{+}, \varphi_{1}^{+}\right):=\mathcal{L}^{-1}\left(\varphi(0), \partial_{\tau} \varphi(0)\right) .
$$

We remark that we may just solve (III.39) on some interval $[T, \infty)$ and then we consider the unique solution of the Cauchy problem on $[0, T]$ with initial data at $T$ given by $\left(\varphi(T), \partial_{\tau} \varphi(T)\right)$. We introduce the Banach space $X_{T}$ of the bounded continuous $H^{1} \times L^{2}$-valued functions on $[T, \infty)$. Thanks to (III.30) and (III.37), the map

$$
\mathcal{G}:(\varphi, \psi) \in X_{T} \longmapsto \mathcal{G}(\varphi, \psi)(\tau):=U(\tau, 0)\left(\psi_{0}^{+}, \psi_{1}^{+}\right)+C \int_{\tau}^{\infty}[\alpha(\sigma)]^{\nu} U(\tau, \sigma)\left(0, \varphi(\sigma)|\varphi(\sigma)|^{p-1}\right) d \sigma
$$

is well defined. Moreover if $T$ is large enough, $\mathcal{G}$ is a strict contraction on

$$
\left\{(\varphi, \psi) \in X_{T} ; \sup _{\tau \geqslant T}\|(\varphi(\tau), \psi(\tau))\|_{H^{1} \times L^{2}} \leqslant 2 \sup _{\tau \geqslant T}\left\|U(\tau, 0)\left(\psi_{0}^{+}, \psi_{1}^{+}\right)\right\|_{H^{1} \times L^{2}}\right\} .
$$

The fixed point $(\varphi, \psi)=\mathcal{G}(\varphi, \psi)$ satisfies $\psi=\partial_{\tau} \varphi$ and $\varphi$ is a solution of (III.39) on [T, $\left.\infty\right)$. Finally we extend $\varphi$ into a solution on $[0, \infty)$ by solving the Cauchy problem with initial data given at time $T$ by $\left(\varphi(T), \partial_{\tau} \varphi(T)\right)$. Therefore we have constructed a solution of (III.39) and $W_{+}^{-1}$ is well defined by (III.40). We remark that $T$ only depends on $\left\|\left(\psi_{0}^{+}, \psi_{1}^{+}\right)\right\|_{H^{1} \times L^{2}}$. Therefore we conclude with (III.30) and (III.33) that there exists a continuous function $K$ such that

$$
\sup _{\tau \geqslant 0}\left(\left\|\partial_{\tau} \varphi(\tau)\right\|_{L^{2}}+\|\varphi(\tau)\|_{H^{1}}\right) \leqslant K\left(\left\|\psi_{0}^{+}\right\|_{H^{1}}+\left\|\psi_{1}^{+}\right\|_{L^{2}}\right)
$$

To establish the Lipchitz properties of $W_{+}$and $W_{+}^{-1}$ we write

$\left(\varphi_{0}^{+}, \varphi_{1}^{+}\right)-\left(\hat{\varphi}_{0}^{+}, \hat{\varphi}_{1}^{+}\right)=\left(\varphi_{0}-\hat{\varphi}_{0}, \varphi_{1}-\hat{\varphi}_{1}\right)-C \int_{0}^{\infty}[\alpha(\sigma)]^{\nu} U(0, \sigma)\left(0, \varphi(\sigma)|\varphi(\sigma)|^{p-1}-\hat{\varphi}(\sigma)|\hat{\varphi}(\sigma)|^{p-1}\right) d \sigma$,

then (III.23) and (III.24) follow from (II.19), (III.30), (III.35), (III.41) and (III.37).

Q.E.D.

\section{At a Big Rip (COnformal COUpling)}

In this part we investigate the behaviour of the solutions of the linear Klein-Gordon equation with a conformal coupling (I.4), (I.16), if a Big Rip occurs at $t_{+}$, that is to say we assume that the scale factor satisfies (I.2) and (I.3) with $\eta_{0}^{+}<0$. Using the conformal time $\tau$ we deal with the Klein-Gordon equation (II.26) with the variable mass $m \alpha(\tau)$ that tends to infinity as $\tau \rightarrow \tau_{+}$. More precisely (IX.1) assures that for some $C>0$,

$$
\alpha(\tau) \sim C\left(\tau_{+}-\tau\right)^{\frac{\eta_{0}^{+}}{1-\eta_{0}^{+}}} .
$$

We remark that the potential $m^{2} \alpha^{2}(\tau)$ in (II.26) belongs to $L^{1}\left(0, \tau_{+}\right)$iff $-1<\eta_{0}^{+}<0$ (Slow Big Rip). We start by considering this case. 
Theorem IV.1 (Slow Big Rip). We assume that (I.2), (I.3) and (II.3) hold. We suppose that $-1<\eta_{0}^{+}<0$. Given $\theta \in[0,1], u_{0} \in H^{1-\theta}(\mathbf{K}), u_{1} \in H^{-\theta}(\mathbf{K})$, we consider the unique $u \in$ $C^{0}\left(I ; H^{1-\theta}(\mathbf{K})\right) \cap C^{1}\left(I ; H^{-\theta}(\mathbf{K})\right)$ solution of (I.4), (I.16), (II.2). Then there exist unique $\varphi_{0}^{+} \in$ $H^{1-\theta}(\mathbf{K}), \varphi_{1}^{+} \in H^{-\theta}(\mathbf{K})$, such that

$$
\left\|[a(t)]^{\frac{d-1}{2}} u(t, .)-\varphi_{0}^{+}\right\|_{H^{1-\theta}}+\left\|a(t) \partial_{t}\left[[a(t)]^{\frac{d-1}{2}} u(t, .)\right]-\varphi_{1}^{+}\right\|_{H^{-\theta}} \longrightarrow 0, \quad t \rightarrow t_{+},
$$

and the map $W_{+}:\left(u_{0}, u_{1}\right) \mapsto\left(\varphi_{0}^{+}, \varphi_{1}^{+}\right)$is an isomorphism on $H^{1-\theta}(\mathbf{K}) \times H^{-\theta}(\mathbf{K})$.

Proof. The proof is similar to those of the case $0 \leqslant \eta_{0}^{+}<1$ but $\alpha$ is no more continuous on $\left[0, \tau_{+}\right]$. Nevertheless thanks to (IV.1), we have $\alpha^{2}(\tau) \in L^{1}\left(0, \tau_{+}\right)$therefore we obtain (III.10) from (III.9) again, and $\left(\varphi_{0}^{+}, \varphi_{1}^{+}\right)$is well defined by (III.11). Conversely, given $\left(\varphi_{0}^{+}, \varphi_{1}^{+}\right) \in H^{1-\theta} \times H^{-\theta}$, we can easily solve the integral equation (III.12) for $\tau \in\left[\tau_{0}, \tau_{+}\right]$for some $\tau_{0}<\tau_{+}$. It is sufficient to take $\tau_{+}-\tau_{0}$ small enough in order the right member of (III.12) to be a strict contraction on $C^{0}\left(\left[\tau_{0}, \tau_{+}\right] ; H^{1-\theta} \times H^{-\theta}\right)$. Then $\left(\varphi(0), \partial_{\tau} \varphi(0)\right.$ is obtained by unique continuation.

Q.E.D.

If $\eta_{0}^{+} \leqslant-1$ we may not expect such a result. Here are some examples showing that $W_{+}$cannot be defined as an isomorphism, more precisely, in these cases, $\varphi(\tau)$ has a limit as $\tau \rightarrow \tau_{+}$but in contrast, $\partial_{\tau} \varphi(\tau)$ is diverging. We suppose that $\mathbf{K}$ is a compact manifold and we assume that there exists $\Phi_{0} \in L^{2}(\mathbf{K})$ solution of $-\Delta_{\mathbf{K}} \Phi_{0}+\frac{d-1}{4 d} R_{\gamma} \Phi_{0}=0$ (for instance, $\mathbf{K}$ is a compact flat manifold and $\left.\Phi_{0}=1\right)$. Then the solution $\varphi$ of $\left[\partial_{\tau}^{2}-\Delta_{\mathbf{K}}+m^{2} \alpha^{2}(\tau)+\frac{d-1}{4 d} R_{\gamma}\right] \varphi=0, \varphi(0)=c_{0} \Phi_{0}$, $\partial_{\tau} \varphi(0)=c_{1} \Phi_{0}$, is given by $\varphi(\tau, \mathbf{x})=u(\tau) \Phi_{0}(\mathbf{x})$ where $u$ is a solution of the differential equation

$$
u^{\prime \prime}+m^{2} \alpha^{2}(\tau) u=0 .
$$

First we investigate the case $a(t)=2\left(t_{+}-t\right)^{-1}$. We have

$$
\alpha(\tau)=\left(\tau_{+}-\tau\right)^{-\frac{1}{2}} .
$$

Then the solution of (IV.3) is expressed with Bessel functions,

$$
u(\tau)=\sqrt{\tau_{+}-\tau}\left[a J_{1}\left(2 m \sqrt{\tau_{+}-\tau}\right)+b Y_{1}\left(2 m \sqrt{\tau_{+}-\tau}\right)\right] \Phi_{0}(\mathbf{x}), \quad a, b \in \mathbb{C},
$$

hence as $\tau \rightarrow \tau_{+}$,

$$
\varphi(\tau, \mathbf{x}) \sim-\frac{b}{m \pi} \Phi_{0}(\mathbf{x}), \quad \partial_{\tau} \varphi(\tau, \mathbf{x}) \sim-\frac{m b}{2 \pi} \ln \left(\tau_{+}-\tau\right) \Phi_{0}(\mathbf{x}) .
$$

We remark that the asymptotics are characterized by $b$, which is not sufficient to obtain $c_{0}, c_{1}$. Moreover the time derivative is not bounded and the following limits exist:

$$
\lim _{\tau \rightarrow \tau_{+}} \varphi(\tau) \text { and } \lim _{\tau \rightarrow \tau_{+}}\left(1+\int_{0}^{\tau} \alpha^{2}(\sigma) d \sigma\right)^{-1} \partial_{\tau} \varphi(\tau) \text { in } L^{2}(\mathbf{K}) .
$$

Secondly we investigate the case $a(t)=9\left(t_{+}-t\right)^{-2}$ for which:

$$
\alpha(\tau)=\left(\tau_{+}-\tau\right)^{-\frac{2}{3}} .
$$

Then the solutions $u$ of (IV.3 $)$ are

$$
u(\tau)=a e^{3 m\left(\tau_{+}-\tau\right)^{\frac{1}{3}} i}\left(1-3 m\left(\tau_{+}-\tau\right)^{\frac{1}{3}} i\right)+b e^{-3 m\left(\tau_{+}-\tau\right)^{\frac{1}{3}} i}\left(1+3 m\left(\tau_{+}-\tau\right)^{\frac{1}{3}} i\right), \quad a, b \in \mathbb{C} .
$$

We have the following asymptotics as $\tau \rightarrow \tau_{+}$:

$$
\varphi(\tau, \mathbf{x}) \sim(a+b) \Phi_{0}(\mathbf{x}), \quad \partial_{\tau} \varphi(\tau, \mathbf{x}) \sim-3 m^{2}(a+b)\left(\tau_{+}-\tau\right)^{-\frac{1}{3}} \Phi_{0}(\mathbf{x}) .
$$

Hence the asymptotics are given by $a+b$ that is not sufficient to get $c_{0}, c_{1}$. Moreover (IV.4 is satisfied again. 
Now we choose $a(t)=64\left(t_{+}-t\right)^{-3}$, hence

$$
\alpha(\tau)=\left(\tau_{+}-\tau\right)^{-\frac{3}{4}}
$$

and the solutions of (IV.3) are

$$
\begin{aligned}
u(\tau) & =a\left[2 m\left(\tau_{+}-\tau\right)^{\frac{1}{2}} J_{0}\left(4 m\left(\tau_{+}-\tau\right)^{\frac{1}{4}}\right)-\left(\tau_{+}-\tau\right)^{\frac{1}{4}} J_{1}\left(4 m\left(\tau_{+}-\tau\right)^{\frac{1}{4}}\right)\right] \\
& +b\left[2 m\left(\tau_{+}-\tau\right)^{\frac{1}{2}} Y_{0}\left(4 m\left(\tau_{+}-\tau\right)^{\frac{1}{4}}\right)-\left(\tau_{+}-\tau\right)^{\frac{1}{4}} Y_{1}\left(4 m\left(\tau_{+}-\tau\right)^{\frac{1}{4}}\right)\right], \quad a, b \in \mathbb{C},
\end{aligned}
$$

and we have as $\tau \rightarrow \tau_{+}$

$$
\varphi(\tau, \mathbf{x}) \sim \frac{b}{2 m \pi} \Phi_{0}(\mathbf{x}), \quad \partial_{\tau} \varphi(\tau, \mathbf{x}) \sim-\frac{m b}{\pi}\left(\tau_{+}-\tau\right)^{-\frac{1}{2}} \Phi_{0}(\mathbf{x}) .
$$

Therefore the previous conclusions hold. To avoid the possibility that the eigenvalue zero plays a peculiar role, we consider an eigenfunction $\Phi_{\lambda}$ associated to some eigenvalue $\lambda^{2}>0$ of $-\Delta_{\mathbf{K}}+$ $\frac{d-1}{4 d} R_{\gamma}$, and we take $a(t)=2\left(t_{+}-t\right)^{-1}$. Then the solution $\varphi$ of $\left[\partial_{\tau}^{2}-\Delta_{\mathbf{K}}+m^{2} \alpha^{2}(\tau)+\frac{d-1}{4 d} R_{\gamma}\right] \varphi=$ $0, \varphi(0)=c_{0} \Phi_{\lambda}, \partial_{\tau} \varphi(0)=c_{1} \Phi_{\lambda}$, is given by $\varphi(\tau, \mathbf{x})=u(\tau) \Phi_{\lambda}(\mathbf{x})$ where $u$ is a solution of the ODE

$$
u^{\prime \prime}+\lambda^{2} u+\frac{m^{2}}{\tau_{+}-\tau} u=0 .
$$

The solutions are expressed with Whittaker functions (with the notations of [41]):

$$
u(\tau)=a M_{\mu, \frac{1}{2}}\left(-2 i \lambda\left(\tau_{+}-\tau\right)\right)+b W_{\mu, \frac{1}{2}}\left(-2 i \lambda\left(\tau_{+}-\tau\right)\right), \quad \mu:=\frac{m^{2}}{2 \lambda} i, \quad a, b \in \mathbb{C} .
$$

We deduce that

$$
\varphi(\tau) \sim \frac{b}{\Gamma\left(1-\frac{m^{2}}{2 \lambda} i\right)} \Phi_{\lambda}(\mathbf{x}), \quad \partial_{\tau} \varphi(\tau, \mathbf{x}) \sim-\frac{b m^{2}}{\Gamma\left(1-\frac{m^{2}}{2 \lambda} i\right)} \ln \left(\tau_{+}-\tau\right) \Phi_{\lambda}(\mathbf{x}) .
$$

All these examples show that the following Theorem concerning the Strong Big $\operatorname{Rip}\left(\eta_{0}^{+} \leqslant-1\right)$, is optimal.

Theorem IV.2 (Strong Big Rip). We assume that (I.2), (I.3) and (II.3) hold. We suppose $\eta_{0}^{+} \leqslant$ -1 . Given $\theta \in[0,1], u_{0} \in H^{1-\theta}(\mathbf{K}), u_{1} \in H^{-\theta}(\mathbf{K})$, we consider the unique $u \in C^{0}\left(I ; H^{1-\theta}(\mathbf{K})\right) \cap$ $C^{1}\left(I ; H^{-\theta}(\mathbf{K})\right)$ solution of (I.4), (I.16), (II.2). Then there exists unique $\varphi_{0}^{+} \in H^{1-\theta}(\mathbf{K})$ such that

$$
\begin{gathered}
{[a(t)]^{\frac{d-1}{2}} u(t, .) \rightarrow \varphi_{0}^{+} \text {in } H^{1-\theta}-\text { weak*, } \quad[a(t)]^{\frac{d-1}{2}} u(t, .) \rightarrow \varphi_{0}^{+} \text {in } H^{-\theta} t \rightarrow t_{+},} \\
\left(t_{+}-t\right)^{-\eta_{0}^{+}-1} \partial_{t}\left([a(t)]^{\frac{d-1}{2}} u\right) \in C^{0} \cap L^{\infty}\left(\left[t_{0}, t_{+}\right) ; H^{-\theta}\right) .
\end{gathered}
$$

The proof of the Theorem is based on an elegant method used in [19]. The following Lemmas, an abstract energy estimate and the resolution of the Ricatti equation, are directly inspired by this paper.

Given a densely defined selfadjoint operator $B$ on a Hilbert space $(\mathcal{H},\|\cdot\|)$, satisfying $I d \leqslant B$, we denote $\mathcal{H}^{\theta}, \theta \leqslant 2$, the closure of $\operatorname{Dom}\left(B^{2}\right)$ for the norm $\|u\|_{\theta}:=\left\|B^{\theta} u\right\|$.

Lemma IV.3. Given $A \in C^{1}\left(\left[\tau_{0}, \tau_{+}\right)\right)$and $G \in C^{0}\left(\left[\tau_{0}, \tau_{+}\right) ; \mathcal{L}(\mathcal{H}) \cap \mathcal{L}\left(\mathcal{H}^{1}\right)\right)$, any $u \in C^{0}\left(\left[\tau_{0}, \tau_{+}\right) ; \mathcal{H}^{1-\theta}\right) \cap$ $C^{1}\left(\left[\tau_{0}, \tau_{+}\right) ; \mathcal{H}^{-\theta}\right), \theta \in[0,1]$, solving

$$
\partial_{\tau}^{2} u+B^{2} u+\left(A^{\prime}(\tau)-A^{2}(\tau)\right) u=G(\tau) u,
$$

satisfies for all $\tau \in\left[\tau_{0}, \tau_{+}\right)$

(IV.10)

$\|u(\tau)\|_{1-\theta}+\frac{1}{1+|A(\tau)|}\left\|\partial_{\tau} u(\tau)\right\|_{-\theta} \leqslant 2\left[\left\|u\left(\tau_{0}\right)\right\|_{1-\theta}+\frac{1}{1+\left|A\left(\tau_{0}\right)\right|}\left\|\partial_{\tau} u\left(\tau_{0}\right)\right\|_{-\theta}\right] e^{\int_{\tau_{0}}^{\tau}|A(\sigma)|+\|G(\sigma)\|_{\mathcal{L}(\mathcal{H}) d \sigma}}$. 
Proof. We remark that thanks to the assumptions on $G$, the Cauchy problem is well posed in $\mathcal{H}^{s} \times \mathcal{H}^{s-1}$ for $s \in[0,2]$ therefore it is sufficient to consider $u \in C^{0}\left(\left[\tau_{0}, \tau_{+}\right) ; \mathcal{H}^{2}\right) \cap C^{1}\left(\left[\tau_{0}, \tau_{+}\right) ; \mathcal{H}^{1}\right) \cap$ $C^{2}\left(\left[\tau_{0}, \tau_{+}\right) ; \mathcal{H}\right)$, and the general case follows from the usual argument of density and continuity. Given $\theta \in[0,1]$, we introduce the modified energy

$$
\mathcal{E}(\tau):=\left(\left\|B^{1-\theta} u(\tau)\right\|^{2}+\left\|B^{-\theta}\left(\partial_{\tau} u(\tau)+A(\tau) u(\tau)\right)\right\|^{2}\right)^{\frac{1}{2}} .
$$

We have

$$
\|u(\tau)\|_{1-\theta} \leqslant \mathcal{E}(\tau),\left\|\partial_{\tau} u(\tau)\right\|_{-\theta} \leqslant(1+|A(\tau)|) \mathcal{E}(\tau)
$$

We compute

$$
\begin{aligned}
\mathcal{E}(\tau) \frac{d}{d \tau} \mathcal{E}(\tau) & =A(\tau)\left[-\left\|B^{1-\theta} u(\tau)\right\|^{2}+\left\|B^{-\theta} \partial_{\tau} u(\tau)+A(\tau) B^{-\theta} u(\tau)\right\|^{2}\right] \\
& +\Re<B^{-\theta} \partial_{\tau} u(\tau)+A(\tau) B^{-\theta} u(\tau), B^{-\theta} G(\tau) u(\tau)> \\
& \leqslant|A(\tau)| \mathcal{E}^{2}(\tau)+\|G(\tau)\|_{\mathcal{L}(\mathcal{H})} \mathcal{E}^{2}(\tau) .
\end{aligned}
$$

We deduce from the Gronwall lemma:

$$
\mathcal{E}(\tau) \leqslant \mathcal{E}\left(\tau_{0}\right) e^{\int_{\tau_{0}}^{\tau}|A(\sigma)|+\|G(\sigma)\|_{\mathcal{L}(\mathcal{H})} d \sigma}
$$

Now (IV.10) follows from (IV.12) and (IV.13).

Q.E.D.

Lemma IV.4. Let $V$ be a non-negative, continuous function on $\left[\tau_{1}, \tau_{+}\right)$. We assume that $V(\tau)=$ $O\left(\left|\tau_{+}-\tau\right|^{\gamma}\right)$ with $\gamma>-2$. Then, given $M>1$, there exists $\tau_{0} \in\left[\tau_{1}, \tau_{+}\right)$such that the Ricatti equation

$$
A^{\prime}(\tau)-A^{2}(\tau)=V(\tau), \quad \tau \in\left[\tau_{0}, \tau_{+}\right),
$$

has a unique solution such that $A\left(\tau_{0}\right)=0$, moreover $A$ satisfies

$$
\begin{gathered}
\int_{\tau_{0}}^{\tau} V(\sigma) d \sigma \leqslant A(\tau) \leqslant 2 M \int_{\tau_{0}}^{\tau} V(\sigma) d \sigma . \\
\int_{\tau_{0}}^{\tau_{+}} A(\tau) d \tau \leqslant \frac{1}{2 M} .
\end{gathered}
$$

Proof. Since $V(\tau)=O\left(\left|\tau_{+}-\tau\right|^{\gamma}\right)$ with $\gamma>-2$, we can choose $\tau_{0} \in\left[\tau_{1}, \tau_{+}\right)$such that

$$
\int_{\tau_{0}}^{\tau_{+}}\left(\int_{\tau_{0}}^{\tau} V(\sigma) d \sigma\right) d \tau \leqslant \frac{1}{4 M^{2}}
$$

We iteratively define the sequence $A_{n}(\tau)$ by $A_{0}(\tau)=0$ and

$$
A_{n+1}(\tau)=\int_{\tau_{0}}^{\tau} V(\sigma) d \sigma+\int_{\tau_{0}}^{\tau} A_{n}^{2}(\sigma) d \sigma
$$

We can easily prove by recurrence that for $n \geqslant 1$ we have

$$
\int_{\tau_{0}}^{\tau} V(\sigma) d \sigma \leqslant A_{n}(\tau) \leqslant A_{n+1}(\tau)
$$

Moreover if we assume that

$$
A_{n}(\tau) \leqslant 2 M \int_{\tau_{0}}^{\tau} V(\sigma) d \sigma
$$


we evaluate

$$
\begin{aligned}
A_{n+1}(\tau) & \leqslant \int_{\tau_{0}}^{\tau} V(\sigma) d \sigma+4 M^{2} \int_{\tau_{0}}^{\tau}\left(\int_{\tau_{0}}^{\sigma} V(\rho) d \rho\right)^{2} d \sigma \\
& \leqslant \int_{\tau_{0}}^{\tau} V(\sigma) d \sigma+4 M^{2}\left(\int_{\tau_{0}}^{\tau} V(\rho) d \rho\right)\left(\int_{\tau_{0}}^{\tau_{+}}\left(\int_{\tau_{0}}^{\sigma} V(\rho) d \rho\right) d \sigma\right) \\
& \leqslant 2 M \int_{\tau_{0}}^{\tau} V(\sigma) d \sigma .
\end{aligned}
$$

We conclude that $A_{n}(\tau)$ is an increasing sequence bounded by $2 M \int_{\tau_{0}}^{\tau} V(\sigma) d \sigma$. Therefore $A:=$ $\lim _{n} A_{n}$ satisfies (IV.15) and by the Beppo Levi theorem it is a solution of

$$
A(\tau)=\int_{\tau_{0}}^{\tau} V(\sigma) d \sigma+\int_{\tau_{0}}^{\tau} A^{2}(\sigma) d \sigma
$$

We conclude that $A$ belongs to $C^{\infty}\left(\left[\tau_{0}, \tau_{+}\right)\right)$and it is a solution of (IV.14) (parenthetically the first Dini theorem assures that $A_{n}$ converges locally uniformly to $A$ on $\left[\tau_{0}, \tau_{+}\right)$). Moreover we have

$$
\int_{\tau_{0}}^{\tau_{+}} A(\tau) d \tau \leqslant 2 M \int_{\tau_{0}}^{\tau_{+}}\left(\int_{\tau_{0}}^{\tau} V(\sigma) d \sigma\right) d \tau \leqslant \frac{1}{2 M}
$$

Proof of the Theorem. (IV.1) assures that $\alpha^{2}(\tau)=O\left(\left|\tau_{+}-\tau\right|^{\gamma}\right)$ with $\gamma>-2$. We use the potential $A$ given by the previous Lemma IV.4 with $V(\tau)=m^{2} \alpha^{2}(\tau)$ and we apply Lemma IV.3 with $B^{2}=-\Delta_{\mathbf{K}}+1, G=1-\frac{d-1}{4 d} R_{\gamma}$ to obtain

$$
\varphi \in C^{0} \cap L^{\infty}\left(\left[\tau_{0}, \tau_{+}\right) ; H^{1-\theta}\right), \quad(1+A)^{-1} \partial_{\tau} \varphi \in C^{0} \cap L^{\infty}\left(\left[\tau_{0}, \tau_{+}\right) ; H^{-\theta}\right) .
$$

Since $\alpha^{2}(\tau) \sim C\left(\tau_{+}-\tau\right)^{\frac{2 \eta_{0}^{+}}{1-\eta_{0}^{+}}}$and $\eta_{0}^{+} \leqslant-1$, we get from (IV.15) that

$$
\left(\tau_{+}-\tau\right)^{\frac{\eta_{0}^{+}+1}{\eta_{0}^{+}-1}} \partial_{\tau} \varphi \in C^{0} \cap L^{\infty}\left(\left[\tau_{0}, \tau_{+}\right) ; H^{-\theta}\right),
$$

and we deduce that

$$
\partial_{\tau} \varphi \in L^{1}\left(\left[\tau_{0}, \tau_{+}\right) ; H^{-\theta}\right) .
$$

We conclude with the Corollary 2.1 of 60 ] that

$$
\varphi \in C^{0}\left(\left[\tau_{0}, \tau_{+}\right], H^{-\theta}\right) \cap C_{w}^{0}\left(\left[\tau_{0}, \tau_{+}\right], H^{1-\theta}\right),
$$

where $C_{w}(I, X)$ denotes the space of the $X$-valued functions defined on an interval $I$ that are weakly continuous. Coming back to $u(t)$, (IV.7) follows from (IV.18) and (IV.8) is equivalent to (IV.17).

Q.E.D.

\section{At a Sudden Singularity (Non Conformal Coupling)}

We consider the case of Sudden Singularity, $\eta_{0}^{+}=0, \eta_{1}^{+} \in(0,1) \cup(1, \infty)$, and a general coupling $\xi \in \mathbb{R}, \xi \neq \frac{d-1}{4 d}$. In conformal time, we consider equation (I.7) where thanks to (IX.2) the potential $V \in C^{0}\left(\tau_{-}, \tau_{+}\right)$given by (I.8) satisfies

$$
V(\tau) \sim m^{2}\left(c_{0}^{+}\right)^{2}+\left(\xi-\frac{d-1}{4 d}\right) 2 d \eta_{1}^{+}\left(\eta_{1}^{+}-1\right) c_{0}^{\eta_{1}^{+}-1} c_{1}^{+}\left|\tau-\tau_{+}\right|^{\eta_{1}^{+}-2}, \quad \tau \rightarrow \tau_{+} .
$$

In the case of a Big Brake $\left(\eta_{1}^{+}>1\right)$, we have $V \in L^{1}\left(\left[0, \tau_{+}\right)\right)$and the situation is analogous to those of the conformal coupling. 
Theorem V.1 (Big Brake). We assume that (I.2), (I.3) and (II.3) and hold. We suppose that $\eta_{0}^{+}=0, \eta_{1}^{+}>1$. Given $\theta \in[0,1], u_{0} \in H^{1-\theta}(\mathbf{K}), u_{1} \in H^{-\theta}(\mathbf{K})$, we consider the unique $u \in$ $C^{0}\left(I ; H^{1-\theta}(\mathbf{K})\right) \cap C^{1}\left(I ; H^{-\theta}(\mathbf{K})\right)$ solution of (I.4), (II.2). Then there exist unique $\varphi_{0}^{+} \in H^{1-\theta}(\mathbf{K})$, $\varphi_{1}^{+} \in H^{-\theta}(\mathbf{K})$, such that

$$
\left\|[a(t)]^{\frac{d-1}{2}} u(t, .)-\varphi_{0}^{+}\right\|_{H^{1-\theta}}+\left\|a(t) \partial_{t}\left[[a(t)]^{\frac{d-1}{2}} u(t, .)\right]-\varphi_{1}^{+}\right\|_{H^{-\theta}} \longrightarrow 0, \quad t \rightarrow t_{+},
$$

and the map $W_{+}:\left(u_{0}, u_{1}\right) \mapsto\left(\varphi_{0}^{+}, \varphi_{1}^{+}\right)$is an isomorphism on $H^{1-\theta}(\mathbf{K}) \times H^{-\theta}(\mathbf{K})$.

Proof. The proof is similar to those of the case $-1<\eta_{0}^{+}<1$ with $\xi=\frac{d-1}{4 d}$ since (V.1) assures that $V \in L^{1}\left(0, \tau_{+}\right)$if $\eta_{1}^{+}>1$. We follow the proof of the Theorem IV.1 by replacing $m^{2} \alpha^{2}(\tau)$ by $V(\tau)$.

Q.E.D.

The case of a Sudden Singularity with $\eta_{1}^{+} \in(0,1)$, is analogous to the Strong Big Rip $\left(\eta_{0}^{+} \leqslant-1\right)$ with the conformal coupling $\xi=\frac{d-1}{4 d}$ since $V(\tau)=O\left(\left|\tau-\tau_{+}\right|^{\gamma}\right), \gamma>-2$.

Theorem V.2 (Sudden Singularity). We assume that (I.2), (I.3) and (II.3) and hold. We suppose $\eta_{0}^{+}=0, \eta_{1}^{+} \in(0,1)$. Given $\theta \in[0,1], u_{0} \in H^{1-\theta}(\mathbf{K}), u_{1} \in H^{-\theta}(\mathbf{K})$, we consider the unique $u \in C^{0}\left(I ; H^{1-\theta}(\mathbf{K})\right) \cap C^{1}\left(I ; H^{-\theta}(\mathbf{K})\right)$ solution of (I.4), (II.2). Then there exists unique $\varphi_{0}^{+} \in$ $H^{1-\theta}(\mathbf{K})$ such that

$$
\begin{gathered}
{[a(t)]^{\frac{d-1}{2}} u(t, .) \rightarrow \varphi_{0}^{+} \text {in } H^{1-\theta}-\text { weak*, } \quad[a(t)]^{\frac{d-1}{2}} u(t, .) \rightarrow \varphi_{0}^{+} \text {in } H^{-\theta} t \rightarrow t_{+},} \\
\left(t_{+}-t\right)^{1-\eta_{1}^{+}} \partial_{t}\left([a(t)]^{\frac{d-1}{2}} u\right) \in C^{0} \cap L^{\infty}\left(\left[t_{0}, t_{+}\right) ; H^{-\theta}\right) .
\end{gathered}
$$

Proof. If $\xi \neq \frac{d-1}{4 d}$, (V.1) assures that $V(\tau)$ has a constant sign near $\tau_{+}$. If $V(\tau) \geqslant 0$ on some interval $\left[\tau_{1}, \tau_{+}\right)$, we can mimic the proof of the Theorem IV.2 for the Strong Big Rip with the conformal coupling, by replacing $m^{2} \alpha^{2}(\tau)$ by $V(\tau)$. In contrast, if $V(\tau) \leqslant 0$ on some interval $\left[\tau_{1}, \tau_{+}\right)$, we cannot apply Lemma IV.4. Fortunately, we can solve the Riccatti equation for a non-positive right-hand side and Lemma IV.4 is replaced by the following:

Lemma V.3. Let $W$ be a non-negative, continuous function on $\left[\tau_{1}, \tau_{+}\right)$. We assume that $W(\tau)=$ $O\left(\left|\tau_{+}-\tau\right|^{\gamma}\right)$ with $\gamma>-2$. Then, given $M>1$, there exists $\tau_{0} \in\left[\tau_{1}, \tau_{+}\right)$such that the Ricatti equation

$$
A^{\prime}(\tau)-A^{2}(\tau)=-W(\tau), \quad \tau \in\left[\tau_{0}, \tau_{+}\right),
$$

has a unique solution such that $A\left(\tau_{0}\right)=0$, moreover $A$ satisfies

$$
\begin{gathered}
-\int_{\tau_{0}}^{\tau} W(\sigma) d \sigma \leqslant A(\tau) \leqslant-\left(1-\frac{1}{2 M}\right) \int_{\tau_{0}}^{\tau} W(\sigma) d \sigma . \\
\int_{\tau_{0}}^{\tau_{+}}|A(\tau)| d \tau \leqslant \frac{1}{2 M} .
\end{gathered}
$$

Proof. Since $W(\tau)=O\left(\left|\tau_{+}-\tau\right|^{\gamma}\right)$ with $\gamma>-2$, we can choose $\tau_{0} \in\left[\tau_{1}, \tau_{+}\right)$such that

$$
\int_{\tau_{0}}^{\tau_{+}}\left(\int_{\tau_{0}}^{\tau} W(\sigma) d \sigma\right) d \tau \leqslant \frac{1}{2 M}
$$

We iteratively define the sequence $A_{n}(\tau)$ by $A_{0}(\tau)=0$ and

$$
A_{n+1}(\tau)=-\int_{\tau_{0}}^{\tau} W(\sigma) d \sigma+\int_{\tau_{0}}^{\tau} A_{n}^{2}(\sigma) d \sigma .
$$


We obviously have for any $n \geqslant 0$

$$
-\int_{\tau_{0}}^{\tau} W(\sigma) d \sigma \leqslant A_{n}(\tau)
$$

and we prove that for any $n \geqslant 1$,

$$
A_{n}(\tau) \leqslant-\left(1-\frac{1}{2 M}\right) \int_{\tau_{0}}^{\tau} W(\sigma) d \sigma
$$

that follows from the inequalities :

$$
\begin{aligned}
A_{n+1}(\tau) & \leqslant-\int_{\tau_{0}}^{\tau} W(\sigma) d \sigma+\int_{\tau_{0}}^{\tau}\left(\int_{\tau_{0}}^{\sigma} W(\rho) d \rho\right)^{2} d \sigma \\
& \leqslant-\int_{\tau_{0}}^{\tau} W(\sigma) d \sigma+\left(\int_{\tau_{0}}^{\tau} W(\rho) d \rho\right)\left(\int_{\tau_{0}}^{\tau_{+}}\left(\int_{\tau_{0}}^{\sigma} W(\rho) d \rho\right) d \sigma\right) \\
& \leqslant-\left(1-\frac{1}{2 M}\right) \int_{\tau_{0}}^{\tau} W(\sigma) d \sigma .
\end{aligned}
$$

Now for $n \geqslant 1$ we evaluate

$$
\begin{aligned}
\left|A_{n+1}(\tau)-A_{n}(\tau)\right| & \leqslant \sup _{\sigma \in\left[\tau_{0}, \tau\right]}\left|A_{n}(\sigma)-A_{n-1}(\sigma)\right| \int_{\tau_{0}}^{\tau}\left|A_{n}(\sigma)+A_{n-1}(\sigma)\right| d \sigma \\
& \leqslant 2 \sup _{\sigma \in\left[\tau_{0}, \tau\right]}\left|A_{n}(\sigma)-A_{n-1}(\sigma)\right| \int_{\tau_{0}}^{\tau}\left(\int_{\tau_{0}}^{\sigma} W(\rho) d \rho\right) d \sigma \\
& \leqslant \frac{1}{M} \sup _{\sigma \in\left[\tau_{0}, \tau\right]}\left|A_{n}(\sigma)-A_{n-1}(\sigma)\right|
\end{aligned}
$$

We deduce that

$$
\sup _{\sigma \in\left[\tau_{0}, \tau\right]}\left|A_{n}(\sigma)-A_{n-1}(\sigma)\right| \leqslant \frac{1}{M^{n}} \int_{\tau_{0}}^{\tau} W(\sigma) d \sigma .
$$

We conclude that we can define $A$ as

$$
A(\tau):=\lim _{n \rightarrow \infty} A_{n}(\tau)=\sum_{n=0}^{\infty}\left(A_{n+1}(\tau)-A_{n}(\tau)\right),
$$

and $A$ satisfies ( $(\overline{V .5}),(\overline{V .6})$ and (V.7) follows from (V.6) and (V.8).

Q.E.D.

To end the proof of the theorem, we apply the previous Lemma with $W(\tau)=-V(\tau)$, then we use Lemma IV.3 with $B^{2}=-\Delta_{\mathbf{K}}+1, G=1-\frac{d-1}{4 d} R_{\gamma}$, and we achieve the proof as for the Theorem IV.2.

Q.E.D.

\section{At a Big Bang/Crunch/Rip (Non Conformal Coupling)}

We consider the case of a Big Crunch, $\eta_{0}^{+}>0$, or a Big Rip $\eta_{0}^{+}<0$ and a general coupling $\xi \in \mathbb{R}, \xi \neq \frac{d-1}{4 d}$. Using the conformal time, we have to investigate the solutions of the linear Klein Gordon equation (I.7) with the potential $V(\tau)$ given by (I.8). The estimates of the scale factor (IX.1) (IX.3) and (IX.4) assure that:

- if $\eta_{0}^{+}>1$, we have

$$
V(\tau)=\left(\xi-\frac{d-1}{4 d}\right) \frac{d \eta_{0}^{+}}{\left(\eta_{0}^{+}-1\right)^{2}}\left(\eta_{0}^{+}(d+1)-2\right) \tau^{-2}+O\left(\tau^{-\frac{2 \eta_{0}^{+}}{\eta_{0}^{+}-1}}\right), \quad \tau \rightarrow+\infty,
$$


- if $\eta_{0}^{+}=1$, we have

$$
V(\tau)=\left(c_{0}^{+}\right)^{2} d(d-1)\left(\xi-\frac{d-1}{4 d}\right)+O\left(e^{-c_{0}^{+} \tau}\right), \quad \tau \rightarrow+\infty,
$$

- if $\eta_{0}^{+}<1$, we have for some $\epsilon>0$

$$
V(\tau)=\left(\xi-\frac{d-1}{4 d}\right)\left[\eta_{0}^{+}(d+1)-2\right] d \frac{\eta_{0}^{+}}{1-\eta_{0}^{+}}\left(\tau_{+}-\tau\right)^{-2}+O\left(\left(\tau_{+}-\tau\right)^{-2+\epsilon}\right), \quad \tau \rightarrow \tau_{+} .
$$

We begin with the case of a $C^{1}-\operatorname{Big}$ Crunch $\left(\eta_{0} \geqslant 1\right)$ for which $\tau_{+}=+\infty$. If $\eta_{0}>1$, $V \in L^{1}([0, \infty))$ hence the situation is analogous to those of the conformal coupling and we compare the solutions of (I.7) with those of (I.10). If $\eta_{0}=1$, the asymptotic dynamics is defined by (I.11). Like for the conformal coupling, we have to avoid the increasing solutions by introducing a spectral cut-off. The Kato Rellich theorem and (II.3) assure that $-\Delta_{\mathbf{K}}+\xi R_{\gamma}$ endowed with the domain $H^{2}(\mathbf{K})$ is selfadjoint on $L^{2}$. Given $\delta \in \mathbb{R}$, we introduce

$$
H_{\xi}^{2, \delta}(\mathbf{K}):=\left\{\varphi \in H^{2}(\mathbf{K}) ; \chi_{(-\infty, \delta)}\left(-\Delta_{\mathbf{K}}+\xi R_{\gamma}\right) \varphi=0\right\}
$$

where $\chi_{\Omega}$ is the characteristic function of $\Omega \subset \mathbb{R}$, and for $s \leqslant 2$ we define $H_{\xi}^{s, \delta}(\mathbf{K})$ as the closure of $H_{\xi}^{2, \delta}(\mathbf{K})$ for the norm (II.4).

Theorem VI.1 ( $C^{1}$-Big Crunch). We assume that (I.2), (I.3) and (II.3) hold with $1 \leqslant \eta_{0}^{+}$. Given $\xi \in \mathbb{R}$, we take $\delta \in \mathbb{R}$ such that

$$
\delta>0 \text { if } \eta_{0}^{+}>1, \quad \delta>-\left(c_{0}^{+}\right)^{2} d(d-1)\left(\xi-\frac{d-1}{4 d}\right) \quad \text { if } \eta_{0}^{+}=1 .
$$

For any $\theta \in[0,1], u_{0} \in H_{\xi}^{1-\theta, \delta}(\mathbf{K}), u_{1} \in H_{\xi}^{-\theta, \delta}(\mathbf{K})$, we consider the unique $u \in C^{0}\left(I ; H^{1-\theta}(\mathbf{K})\right) \cap$ $C^{1}\left(I ; H^{-\theta}(\mathbf{K})\right)$ solution of $(I .4)$, (II.2). Then there exist unique $\varphi_{0}^{+} \in H_{\xi}^{1-\theta, \delta}(\mathbf{K}), \varphi_{1}^{+} \in H_{\xi}^{-\theta, \delta}(\mathbf{K})$, such that

$$
\left\|[a(t)]^{\frac{d-1}{2}} u(t, .)-\varphi(\tau)\right\|_{H^{1-\theta}}+\left\|a(t) \partial_{t}\left[[a(t)]^{\frac{d-1}{2}} u(t, .)\right]-\partial_{\tau} \varphi(\tau)\right\|_{H^{-\theta}} \longrightarrow 0, \quad t \rightarrow t_{+},
$$

where $\varphi$ is the solution of (I.10) if $\eta_{0}^{+}>1$ or (I.11) if $\eta_{0}^{+}=1$, satisfying $\varphi(0)=\varphi_{0}^{+}, \partial_{\tau} \varphi(0)=\varphi_{1}^{+}$. The map $W_{+}:\left(u_{0}, u_{1}\right) \mapsto\left(\varphi_{0}^{+}, \varphi_{1}^{+}\right)$is an isomorphism on $H_{\xi}^{1-\theta, \delta}(\mathbf{K}) \times H_{\xi}^{-\theta, \delta}(\mathbf{K})$.

If $\theta=\frac{1}{2}$ and $\sigma$ is given by (II.6), we have

$$
\sigma(u, \tilde{u})=\left\langle\varphi_{0}^{+}, \tilde{\varphi}_{1}^{+}\right\rangle-\left\langle\tilde{\varphi}_{0}^{+}, \varphi_{1}^{+}\right\rangle
$$

where $\left(\tilde{\varphi}_{0}^{+}, \tilde{\varphi}_{1}^{+}\right)=W_{+}\left(\tilde{u}\left(t_{0}\right), \partial_{t} \tilde{u}\left(t_{0}\right)\right)$.

Proof. The proof follows the same way used for the conformal coupling. If $\eta_{0}^{+}>1$ we just replace $m^{2} \alpha^{2}(\tau)$ by $V(\tau)$ taking advantage of its integrability. If $\eta_{0}^{+}=1$ we replace $U_{0}(\tau)$ by the propagator of (I.11) that is uniformly bounded on $H^{1-\theta, \delta} \times H^{-\theta, \delta}$, and we get the existence and asymptotic completeness of $W_{+}$by using the integrability of $V(\tau)-\left(c_{0}^{+}\right)^{2} d(d-1)\left(\xi-\frac{d-1}{4 d}\right)$.

Q.E.D.

For a $C^{0}-$ Big Crunch or a Big Rip with a non conformal coupling, the potential $V(\tau)$ has a singularity of $\left(\tau_{+}-\tau\right)^{-2}$ type that forbids the existence of $W_{+}$.

Theorem VI.2 $\left(C^{0}\right.$-Big Crunch, Big Rip). We assume that (I.2), (I.3) and (II.3) hold with $\eta_{0}^{+} \in(-\infty, 0) \cup(0,1)$. Given $\xi \in \mathbb{R}$ satisfying (I.18), $\theta \in[0,1]$, there exists $K>0$ such that for any $u_{0} \in H^{1-\theta}(\mathbf{K}), u_{1} \in H^{-\theta}(\mathbf{K})$, the unique solution $u \in C^{0}\left(I ; H^{1-\theta}(\mathbf{K})\right) \cap C^{1}\left(I ; H^{-\theta}(\mathbf{K})\right)$ of (I.4), (II.2) satisfies:

$$
\left(t_{+}-t\right)^{\frac{d-1}{2} \eta_{0}^{+}}\|u(t, .)\|_{H^{1-\theta}} \longrightarrow 0, \quad t \rightarrow t_{+},
$$


(VI.6)

$\sup _{t \in\left[t_{0}, t\right)}\left(\left(t_{+}-t\right)^{\frac{d-1}{2}} \eta_{0}^{+}\|u(t, .)\|_{H^{1-\theta}}+\left(t_{+}-t\right)^{\eta_{0}^{+}+1-\epsilon}\left\|\partial_{t}\left[[a(t)]^{\frac{d-1}{2}} u(t, .)\right]\right\|_{H^{-\theta}}\right) \leqslant K\left(\left\|u_{0}\right\|_{H^{1-\theta}}+\left\|u_{1}\right\|_{H^{-\theta}}\right)$.

Remark VI.3. Due to constraint (I.18), this theorem cannot be applied to the important case $d=3, \xi=0, \eta_{0}^{+} \in(0,1)$. This situation, with supplementary hypotheses, can be treated in the general framework developped by $H$. Ringström for the silent singularities of Bianchi spacetimes [50], [51]. We assume that: (1) $\mathbf{K}$ is a connected 3-dimensional Lie group (an elementary but interesting example is $\mathbf{K}=S^{3}=S U(2)$ ) and the mean curvature of $\mathbf{K} \times\{t\}$ is strictly positive; (2) $\eta_{0}^{+} \in(1 / 3,1)$ (therefore $t_{+}$is a "silent monotone volume singularity"). Then the main results of [50] imply that the solution $u$ of (II.1) with $m=0, \xi=0$ satisfies

$$
u(t) \sim A\left(t_{+}-t\right)^{1-3 \eta_{0}^{+}}+B,\left(t_{+}-t\right)^{3 \eta_{0}^{+}} \partial_{t} u(t) \rightarrow C, \quad t \rightarrow t_{+} .
$$

These asymptotics are also obtained by A. Alho, G. Fournodavlos and A. T. Franzen in [1] if $\mathbf{K}=\mathbb{R}^{3}, \eta_{0}^{+} \in(1 / 3,1)$.

Proof. First we write the Klein-Gordon equation (I.7) as:

$$
\left\{\partial_{\tau}^{2}+\mathfrak{A}+\frac{q}{\left(\tau_{+}-\tau\right)^{2}}\right\} \varphi=p(\tau) \varphi
$$

where

$$
\begin{gathered}
q:=\left(\xi-\frac{d-1}{4 d}\right)\left[\eta_{0}^{+}(d+1)-2\right] d \frac{\eta_{0}^{+}}{1-\eta_{0}^{+}}>\frac{1}{4}, \\
p(\tau):=N+\frac{q}{\left(\tau_{+}-\tau\right)^{2}}-V(\tau),
\end{gathered}
$$

and $N \geqslant 0$ is choosen such that

$$
\mathfrak{A}:=-\Delta_{\mathbf{K}}+\xi R_{\gamma}(\mathbf{x})+N \geqslant I d .
$$

We remark that (IX.1) assures that $p \in C^{0}\left(\left[0, \tau_{+}\right)\right)$satisfies for some $\epsilon \in\left(0, \frac{1}{2}\right)$ and $C_{p}>0$,

$$
|p(\tau)| \leqslant C_{p}\left(\tau_{+}-\tau\right)^{-2+\epsilon}
$$

Using the spectral decomposition of $\mathfrak{A}$, the issue of the asymptotics of $\varphi$ will be reduced to the study of the behaviour at $\tau_{+}$of the solution of the equation

$$
\psi^{\prime \prime}(\tau)+\lambda^{2} \psi(\tau)+\frac{q}{\left(\tau_{+}-\tau\right)^{2}} \psi(\tau)=p(\tau) \psi(\tau), \quad \tau \in\left[0, \tau_{+}\right)
$$

Lemma VI.4. Given $q>\frac{1}{4}, M>0$, there exists $K>0$ such as for any $\lambda \geqslant M, \psi_{0}, \psi_{1} \in \mathbb{C}$, the solution $\psi \in C^{0}\left(\left[0, \tau_{+}\right)\right)$of (VI.8) with intial data $\psi(0)=\psi_{0}, \psi^{\prime}(0)=\psi_{1}$, satisfies

$$
\sup _{0 \leqslant \tau<\tau_{+}}\left(\lambda|\psi(\tau)|+\left(\tau_{+}-\tau\right)^{1-\epsilon}\left|\partial_{\tau} \psi(\tau)\right|\right) \leqslant K\left(\lambda\left|\psi_{0}\right|+\left|\psi_{1}\right|\right) .
$$

Moreover we have

$$
\psi(\tau) \rightarrow 0, \tau \rightarrow \tau_{+} .
$$

Proof. Given $\lambda>0, q>\frac{1}{4}$, we consider the solution $\psi$ of

$$
\psi^{\prime \prime}(\tau)+\lambda^{2} \psi(\tau)+\frac{q}{\left(\tau_{+}-\tau\right)^{2}} \psi(\tau)=0, \quad \tau \in\left[0, \tau_{+}\right), \quad \psi(0)=\psi_{0}, \quad \psi^{\prime}(0)=\psi_{1} .
$$

$\psi$ is expressed by using the Riemann functions $R_{k}(\tau ; \lambda)$, where $R_{0}$ is the solution for $\psi_{0}=1$, $\psi_{1}=0$, and $R_{1}$ is the solution for $\psi_{0}=0$ and $\psi_{1}=1$,

$$
\psi(\tau)=R_{0}(\tau ; \lambda) \psi_{0}+R_{1}(\tau ; \lambda) \psi_{1} .
$$


$R_{k}$ can be written in terms of Bessel functions. Writing $\psi(\tau)=\left(\tau_{+}-\tau\right)^{\frac{1}{2}} f\left(\lambda\left(\tau_{+}-\tau\right)\right)$, we can see that $\psi$ is a solution of (VI.11) iff $f(z)$ is solution of the Bessel equation

$$
f^{\prime \prime}(z)+\frac{1}{z} f^{\prime}(z)+\left(1+\frac{\mu^{2}}{z^{2}}\right)=0, \quad \mu:=\sqrt{q-\frac{1}{4}} .
$$

Elementary and tedious computations give

$$
\begin{gathered}
R_{0}(\tau ; \lambda)=\left(\tau_{+}-\tau\right)^{\frac{1}{2}} \frac{\pi}{4} \tau_{+}^{-1}\left[\left(2 \lambda \tau_{+} Y_{i \mu}^{\prime}\left(\lambda \tau_{+}\right)+Y_{i \mu}\left(\lambda \tau_{+}\right)\right) J_{i \mu}\left(\lambda\left(\tau_{+}-\tau\right)\right)\right. \\
\left.-\left(2 \lambda \tau_{+} J_{i \mu}^{\prime}\left(\lambda \tau_{+}\right)+J_{i \mu}\left(\lambda \tau_{+}\right)\right) Y_{i \mu}\left(\lambda\left(\tau_{+}-\tau\right)\right)\right], \\
R_{1}(\tau ; \lambda)=\left(\tau_{+}-\tau\right)^{\frac{1}{2}} \frac{\pi}{2} \tau_{+}^{\frac{1}{2}}\left[J_{i \mu}\left(\lambda \tau_{+}\right) Y_{i \mu}\left(\lambda\left(\tau_{+}-\tau\right)\right)-Y_{i \mu}\left(\lambda \tau_{+}\right) J_{i \mu}\left(\lambda\left(\tau_{+}-\tau\right)\right)\right] .
\end{gathered}
$$

We estimate $R_{k}(\tau ; \lambda)$ near $\tau_{+}$. A characteristic time is given by

$$
\tau_{\lambda}:=\left(1-M \lambda^{-1}\right) \tau_{+} .
$$

We know (see [41]) that $J_{i \mu}(x), Y_{i \mu}(x), x J_{i \mu}^{\prime}(x), x Y_{i \mu}^{\prime}(x)$ are bounded on $(0,1)$ and $J_{i \mu}(x), J_{i \mu}^{\prime}(x)$, $Y_{i \mu}(x), Y_{i \mu}^{\prime}(x)$ decay as $x^{-\frac{1}{2}}$ as $x \rightarrow \infty$. Therefore, given $M>0$, there exists $C>0$ such that for any $\lambda \geqslant M$ we have for $k=0,1$,

$$
\begin{gathered}
\forall \tau \in\left[0, \tau_{\lambda}\right],\left|R_{k}(\tau ; \lambda)\right|+\lambda^{-1}\left|\partial_{\tau} R_{k}(\tau)\right| \leqslant C \lambda^{-k}, \\
\forall \tau \in\left[\tau_{\lambda}, \tau_{+}\right),\left|R_{k}(\tau ; \lambda)\right|+\left(\tau_{+}-\tau\right)\left|\partial_{\tau} R_{k}(\tau)\right| \leqslant C \lambda^{\frac{1}{2}-k}\left(\tau_{+}-\tau\right)^{\frac{1}{2}},
\end{gathered}
$$

(see [19] for another proof using the confluent hyperbolic functions). The solution $\psi \in C^{0}\left(\left[0, \tau_{+}\right)\right)$ of (VI.8) is given by

$$
\psi(\tau)=R_{0}(\tau ; \lambda) \psi_{0}+R_{1}(\tau ; \lambda) \psi_{1}+\int_{0}^{\tau} K_{\lambda}(\tau, \sigma) p(\sigma) \psi(\sigma) d \sigma
$$

where we have put

$$
K_{\lambda}(\tau, \sigma):=R_{1}(\tau ; \lambda) R_{0}(\sigma ; \lambda)-R_{0}(\tau ; \lambda) R_{1}(\sigma ; \lambda), \quad 0 \leqslant \sigma, \tau<\tau_{+} .
$$

Thanks to (VI.7) and (VI.14) we have for $\tau \in\left[0, \tau_{\lambda}\right]:$ we have

$$
0 \leqslant \sigma \leqslant \tau,\left|K_{\lambda}(\tau, \sigma) p(\sigma)\right| \leqslant 2 C^{2} C_{p} \lambda^{-1}\left(\tau_{+}-\sigma\right)^{-2+\epsilon} \leqslant 2 C^{2} C_{p} M^{-1}\left(\tau_{+}-\sigma\right)^{-1+\epsilon},
$$

and with (VI.15) for $\tau \in\left[\tau_{\lambda}, \tau_{+}\right)$:

$$
0 \leqslant \sigma \leqslant \tau_{\lambda}, \quad\left|K_{\lambda}(\tau, \sigma) p(\sigma)\right| \leqslant 2 C^{2} C_{p} \lambda^{-\frac{1}{2}}\left(\tau_{+}-\tau\right)^{\frac{1}{2}}\left(\tau_{+}-\sigma\right)^{-2+\epsilon} \leqslant 2 C^{2} C_{p} M^{-\frac{1}{2}}\left(\tau_{+}-\sigma\right)^{-1+\epsilon},
$$

$$
\tau_{\lambda} \leqslant \sigma \leqslant \tau,\left|K_{\lambda}(\tau, \sigma) p(\sigma)\right| \leqslant 2 C^{2} C_{p}\left(\tau_{+}-\tau\right)^{\frac{1}{2}}\left(\tau_{+}-\sigma\right)^{-\frac{3}{2}+\epsilon} \leqslant 2 C^{2} C_{p}\left(\tau_{+}-\sigma\right)^{-1+\epsilon} .
$$

We deduce that there exists $C^{\prime}>0$ independent of $\lambda \geqslant M$ such that for any $\tau \in\left[0, \tau_{+}\right)$

$$
|\psi(\tau)| \leqslant C^{\prime}\left[\left|\psi_{0}\right|+\lambda^{-1}\left|\psi_{1}\right|+\int_{0}^{\tau}\left(\tau_{+}-\sigma\right)^{-1+\epsilon}|\psi(\sigma)| d \sigma\right] .
$$

Then the Gronwall lemma implies that

$$
\forall \tau \in\left[0, \tau_{+}\right),|\psi(\tau)| \leqslant C^{\prime}\left(\left|\psi_{0}\right|+\lambda^{-1}\left|\psi_{1}\right|\right) \exp \left(C^{\prime} \epsilon^{-1} \tau_{+}^{\epsilon}\right) .
$$

Moreover since $R_{k}(\tau ; \lambda)$ and $K_{\lambda}(\tau, \sigma)$ tend to zero as $\tau \rightarrow \tau_{+}$, (VI.10) follows from the dominated convergence theorem applied to (VI.16). To obtain the estimate for $\partial_{\tau} \psi$ we note that

$$
\begin{gathered}
0 \leqslant \sigma \leqslant \tau \leqslant \tau_{\lambda},\left|\partial_{\tau} K(\tau, \sigma)\right| \leqslant 2 C^{2} \\
0 \leqslant \sigma \leqslant \tau_{\lambda} \leqslant \tau<\tau_{+}, \quad\left|\partial_{\tau} K(\tau, \sigma)\right| \leqslant 2 C^{2} \lambda^{-\frac{1}{2}}\left(\tau_{+}-\tau\right)^{-\frac{1}{2}}, \\
\tau_{\lambda} \leqslant \sigma \leqslant \tau<\tau_{+}, \quad\left|\partial_{\tau} K(\tau, \sigma)\right| \leqslant 2 C^{2}\left(\tau_{+}-\tau\right)^{-\frac{1}{2}}\left(\tau_{+}-\sigma\right)^{\frac{1}{2}},
\end{gathered}
$$


and we deduce from (VI.7), (VI.16) and (VI.21) that there exists $C^{\prime \prime}>0$ independent of $\lambda \geqslant M$ such that

$$
\forall \tau \in\left[0, \tau_{+}\right), \quad\left|\partial_{\tau} \psi(\tau)\right| \leqslant C^{\prime \prime}\left(\lambda\left|\psi_{0}\right|+\left|\psi_{1}\right|\right)\left(\tau_{+}-\tau\right)^{-1+\epsilon}
$$

The spectral theorem assures the existence of a measure space $(\mathbf{M}, d \mu)$ with finite measure $d \mu$, an unitary operator $U$ from $L^{2}(\mathbf{K})$ onto $L^{2}(\mathbf{M}, d \mu)$ and a real valued function $f \in \bigcap_{p \geqslant 1} L^{p}(\mathbf{M}, d \mu)$ such that

$$
\psi \in D(\mathfrak{A}) \Leftrightarrow f(.)(U \psi)(.) \in L^{2}(\mathbf{M}, d \mu), \quad(U(\mathfrak{A} \psi))(\mu)=f(\mu)(U \psi)(\mu)
$$

Since $\mathfrak{A} \geqslant I d$, we have $f \geqslant 1$ a.e. and on $D(\mathfrak{A})$ the norms $H^{s}(\mathbf{K})$ are equivalent to

$$
\left(\int_{\mathbf{M}}|(U \psi)(\mu)|^{2}[f(\mu)]^{s} d \mu\right)^{\frac{1}{2}} .
$$

Therefore, for $s \geqslant 0$, we may consider $U$ as an isometry from $H^{s}(\mathbf{K})$ onto $L_{s}^{2}(\mathbf{M})$ where $L_{s}^{2}(\mathbf{M}):=$ $L^{2}\left(\mathbf{M},[f(\mu)]^{s} d \mu\right)$. Moreover, $U$ can be uniquely extended into an isometry from $H^{s}(\mathbf{K})$ onto $L_{s}^{2}(\mathbf{M})$ for any $s \leqslant 0$, denoted $U$ again.

For $\mu \in \mathbf{M}$ we introduce the solution $\psi(\tau ; \mu)$ of the equation (VI.8) with the initial data $\psi(0 ; \mu)=$ $\left(U \varphi_{0}\right)(\mu), \partial_{\tau} \psi(0 ; \mu)=\left(U \varphi_{1}\right)(\mu)$ where $\left(\varphi_{0}, \varphi_{1}\right)=\mathcal{L}\left(u_{0}, u_{1}\right)$ given by (II.25). Lemma VI.4 with $\lambda=[f(\mu)]^{\frac{1}{2}}$ and $M=1$ implies that

$$
\begin{gathered}
\psi \in C^{0}\left(\left[0, \tau_{+}\right] ; L_{1-\theta}^{2}(\mathbf{M})\right) \cap C^{1}\left(\left[0, \tau_{+}\right) ; L_{-\theta}^{2}(\mathbf{M})\right), \\
\sup _{\tau \in\left[0, \tau_{+}\right)}\left(\|\psi(\tau ; .)\|_{L_{1-\theta}^{2}}+\left(\tau_{+}-\tau\right)^{1-\epsilon}\left\|\partial_{\tau} \psi(\tau ; .)\right\|_{L_{-\theta}^{2}}\right) \leqslant K\left(\left\|\varphi_{0}\right\|_{H^{1-\theta}}+\left\|\varphi_{1}\right\|_{H^{-\theta}}\right), \\
\psi\left(\tau_{+} ; .\right)=0 .
\end{gathered}
$$

Then $\varphi(\tau, \mathbf{x}):=\left(U^{-1} \psi(\tau ;).\right)(\mathbf{x})$ belongs to $C^{0}\left(\left[0, \tau_{+}\right] ; H^{1-\theta}(\mathbf{K})\right) \cap C^{1}\left(\left[0, \tau_{+}\right) ; H^{-\theta}(\mathbf{K})\right)$ and satisfies

$$
\sup _{\tau \in\left[0, \tau_{+}\right)}\left(\|\varphi(\tau ; .)\|_{H^{1-\theta}}+\left(\tau_{+}-\tau\right)^{1-\epsilon}\left\|\partial_{\tau} \varphi(\tau ; .)\right\|_{H^{-\theta}}\right) \leqslant K\left(\left\|\varphi_{0}\right\|_{H^{1-\theta}}+\left\|\varphi_{1}\right\|_{H^{-\theta}}\right), \quad \varphi\left(\tau_{+}\right)=0 .
$$

Moreover $\varphi$ is solution of (I.7) and $\varphi(0, \mathbf{x})=\varphi_{0}(\mathbf{x}), \partial_{\tau} \varphi(0, \mathbf{x})=\varphi_{1}(\mathbf{x})$. Now the theorem follows from the uniqueness of the solution of the Cauchy problem for (I.4).

Q.E.D.

\section{QuAntum FIELD}

In this section we investigate a peculiar aspect of the quantum fields theory in curved spacetimes: the particle creation in a universe beginning with a Big Bang $\left(\eta_{0}^{-}>0\right)$ and ending with a Big Crunch $\left(\eta_{0}^{+}>0\right)$ or a Sudden Singularity $\left(\eta_{0}^{+}=0\right)$. For the sake of simplicity, we suppose that $\mathbf{K}$ is a compact manifold and the main result of this part states that the number of cosmological particle creation is finite under general assumptions. If $\mathbf{K}$ is a homogeneous infinite space, this number obviously has to be infinite. We let open the issue of the finitness of the local density of particle creation if the volume of $\mathbf{K}$ is infinite.

In the previous sections, we have proved the existence and asymptotic completeness of the wave operators associated to the Klein-Gordon equation (I.7) if the potential $V(\tau)$ defined by (I.8) has finite limits at $\tau_{ \pm}$

$$
V_{ \pm}:=\lim _{\tau \rightarrow \tau_{ \pm}} V(\tau) .
$$

For us to be able to define the (anti-)particles, the asymptotic Hamiltonians

$$
-\Delta_{\mathbf{K}}+\xi R_{\gamma}+V_{ \pm}
$$


have to be positive on a suitable subspace of $L^{2}(\mathbf{K})$. Hence we introduce an infrared cut-off by choosing $\delta \in \mathbb{R}$ such that

$$
\delta>-\min \left(V_{-}, V_{+}\right)
$$

and using (VI.1) we put

$$
X:=H_{\xi}^{\frac{1}{2}, \delta}(\mathbf{K}) \times H_{\xi}^{-\frac{1}{2}, \delta}(\mathbf{K})
$$

that is a Hilbert space for the equivalent norms:

$$
\left\|\left(\varphi_{0}, \varphi_{1}\right)\right\|_{ \pm}:=\left(\left\|\left(-\Delta_{\mathbf{K}}+\xi R_{\gamma}+V_{ \pm}\right)^{\frac{1}{4}} \varphi_{0}\right\|_{L^{2}(\mathbf{K})}^{2}+\left\|\left(-\Delta_{\mathbf{K}}+\xi R_{\gamma}+V_{ \pm}\right)^{-\frac{1}{4}} \varphi_{1}\right\|_{L^{2}(\mathbf{K})}^{2}\right)^{\frac{1}{2}} .
$$

Therefore the classical Scattering Operator

$$
S:=W_{+} \circ W_{-}^{-1}
$$

exists and it is an isomorphism on $X$ in the following cases presented in Figures 4 and 5 ,

\begin{tabular}{|c|c|c|c|}
\hline Singularity & $\eta_{0}^{ \pm}$ & $\tau_{ \pm}$ & $V_{ \pm}$ \\
\hline \hline$C^{0}-$ Big Bang & $0<\eta_{0}^{-}<1$ & $-\infty<\tau_{-}$ & $V_{-}=0$ \\
\hline$C^{1}-$ Big Bang & $1 \leqslant \eta_{0}^{-}$ & $\tau_{-}=-\infty$ & $V_{-}=0$ \\
\hline \hline Sudden Singularity & $\eta_{0}^{+}=0$ & $\tau_{+}<\infty$ & $V_{+}=m^{2}\left(c_{0}^{+}\right)^{2}$ \\
\hline$C^{0}-$ Big Crunch & $0<\eta_{0}^{+}<1$ & $\tau_{+}<\infty$ & $V_{+}=0$ \\
\hline$C^{1}-$ Big Crunch & $1 \leqslant \eta_{0}^{+}$ & $\tau_{+}=\infty$ & $V_{+}=0$ \\
\hline
\end{tabular}

FiguRE 4. Existence of the Scattering Operator for the conformal coupling $\xi=\frac{d-1}{4 d}$.

\begin{tabular}{|c|c|c|c|c|}
\hline Singularity & $\eta_{0}^{ \pm}$ & $\eta_{1}^{+}$ & $\tau_{ \pm}$ & $V_{ \pm}$ \\
\hline \hline$C^{1}-$ Big Bang & $\eta_{0}^{-}=1$ & $\left(\eta_{0}^{-}, \infty\right)$ & $\tau_{-}=-\infty$ & $V_{-}=\left(c_{0}^{-}\right)^{2} d(d-1)\left(\xi-\frac{d-1}{4 d}\right)$ \\
\hline$C^{1}-$ Big Bang & $\eta_{0}^{-}>1$ & $\left(\eta_{0}^{-}, \infty\right)$ & $\tau_{-}=-\infty$ & $V_{-}=0$ \\
\hline \hline$C^{1}-$ Big Brake & $\eta_{0}^{+}=0$ & $\eta_{1}^{+}>2$ & $\tau_{+}<\infty$ & $V_{+}=m^{2}\left(c_{0}^{+}\right)^{2}$ \\
\hline$C^{1}-$ Big Crunch & $\eta_{0}^{+}=1$ & $\left(\eta_{0}^{+}, \infty\right)$ & $\tau_{+}=\infty$ & $V_{+}=\left(c_{0}^{+}\right)^{2} d(d-1)\left(\xi-\frac{d-1}{4 d}\right)$ \\
\hline$C^{1}-$ Big Crunch & $\eta_{0}^{+}>1$ & $\left(\eta_{0}^{+}, \infty\right)$ & $\tau_{+}=\infty$ & $V_{+}=0$ \\
\hline
\end{tabular}

FiguRE 5. Existence of the Scattering Operator for the non conformal coupling $\xi \neq \frac{d-1}{4 d}$.

To quantize the scattering operator we must introduce the one-particle space $X_{\text {pos }}^{ \pm}$and the oneantiparticle $X_{\text {neg }}^{ \pm}$that are defined as

$$
X_{\text {pos }(n e g)}^{ \pm}:=\left\{\left(\varphi_{0}, \varphi_{1}\right) \in X ; \varphi_{1}=+(-) i\left(-\Delta_{\mathbf{K}}+\xi R_{\gamma}+V_{ \pm}\right)^{\frac{1}{2}} \varphi_{0}\right\} .
$$

We denote $P_{\text {pos(neg) }}^{ \pm}$the projection on $X_{\text {pos }(n e g)}^{ \pm}$along $X_{\text {neg(pos })}^{ \pm}$:

$$
P_{p o s(n e g)}^{ \pm}=\frac{1}{2}\left(\begin{array}{cc}
I d & -(+) i\left(-\Delta_{\mathbf{K}}+\xi R_{\gamma}+V_{ \pm}\right)^{-\frac{1}{2}} \\
+(-) i\left(-\Delta_{\mathbf{K}}+\xi R_{\gamma}+V_{ \pm}\right)^{\frac{1}{2}} & I d
\end{array}\right) .
$$

A classic result of the second quantization due to Shale [57] (see so Theorem XI.108 in [45]) assures that $S$ is a Bogolioubov transform that is unitarily implementable on the Fock-Cook spaces over $X$, iff its off diagonal parts defining the particle-antiparticle mixing,

$$
P_{n e g(p o s)}^{+} S P_{p o s(n e g)}^{-}
$$


are Hilbert-Schmidt. The physical meaning of this result is that a finite number $\mathcal{N}$ of creation of particle-antiparticle pairs occurs at $\tau_{+}$if the initial quantum state is the Fock vacuum at $\tau_{-}$. This number is given by

$$
\mathcal{N}=\left\|P_{n e g(\text { pos })}^{+} S P_{\text {pos }(n e g)}^{-}\right\|_{H S}^{2}
$$

where $\|\cdot\|_{H S}$ denotes the Hilbert-Schmidt norm (for explanations of the meaning of this norm in terms of particles, see e.g. [27], [28], 44], 45]).

Adopting the approach introduced by Fulling [28, a key ingredient to prove this property is the Liouville-Green (WKB) approximation that needs a sufficient regularity of the potential. In particular to apply the famous theorems of Olver [43], it is necessary to have $V^{\prime \prime} \in L^{1}\left(\tau_{-}, \tau_{+}\right)$. Hence some supplementary constraints on $\eta_{0}^{ \pm}$and $\eta_{1}^{ \pm}$will be necessary. Moreover the Hilbert-Schmidt property of (VII.3) is closely linked to the convergence of the series of the Zeta function $\zeta(s)$ of the Laplacian $\Delta_{\mathbf{K}}$, that is converging iff $\Re s>d / 2$. Therefore the constraint of regularity of the scale factor is increasing with the dimension $d$ of $\mathbf{K}$. Hence we introduce:

$$
l(d):=\max (2,[d / 2]), \quad[d / 2] \in \mathbb{N}, \quad[d / 2] \leqslant d / 2<[d / 2]+1 .
$$

The following theorem is established by proving that

$$
\mathcal{N} \lesssim \zeta(-l(d)-1):=\sum_{n} \lambda_{n}^{-l(d)-1}
$$

where $\lambda_{n}$ are the positive eigenvalues of $-\Delta_{\mathbf{K}}$. In some cases, the WKB approximation (more precisely, Theorem 4 in [43]) allows to only obtain

$$
\mathcal{N} \lesssim \zeta(-3)=\sum_{n} \lambda_{n}^{-3}
$$

Therefore the constraint of convergence, $3>d / 2$, restricts these results to $d \leqslant 5$.

Theorem VII.1. The operators (VII.3) are Hilbert-Schmidt on $X$ if we assume one of the following hypotheses in which $a \in C^{l}\left(t_{-}, t_{+}\right)$satisfies (I.2) for any $k \leqslant l$.

- A universe beginning with a Big Bang and ending with a Big Crunch:

$$
\begin{gathered}
\xi=\frac{d-1}{4 d}, \quad \frac{l-1}{l+1} \leqslant \eta_{0}^{-}, \eta_{0}^{+}, \quad d \geqslant 3, \quad l=l(d), \\
\xi \neq \frac{d-1}{4 d}, \quad 1<\eta_{0}^{-}, \eta_{0}^{+}, \quad d \geqslant 3, \quad l=l(d)+2, \\
\xi \neq \frac{d-1}{4 d}, \quad \eta_{0}^{-}=\eta_{0}^{+}=1, \quad c_{0}^{-}=c_{0}^{+}, \quad d \geqslant 3, \quad l=l(d)+2, \\
\xi \neq \frac{d-1}{4 d}, \quad 1=\min \left(\eta_{0}^{-}, \eta_{0}^{+}\right), \quad d \leqslant 5, \quad l=4,
\end{gathered}
$$

- A universe beginning with a Big Bang and ending with a Big Brake:

$$
\begin{gathered}
\xi=\frac{d-1}{4 d}, \quad \eta_{0}^{-} \in(1 / 3, \infty), \quad \eta_{0}^{+}=0, \quad \eta_{1}^{+}>1, \quad d \leqslant 5, \quad l=2, \\
\xi \neq \frac{d-1}{4 d}, \quad 1 \leqslant \eta_{0}^{-}, \quad \eta_{0}^{+}=0, \quad \eta_{1}^{+}>3, \quad d \leqslant 5, \quad l=4 .
\end{gathered}
$$


Proof of the Theorem. We shall use the notation $A(\lambda) \sim B(\lambda)$ where $\lambda$ is an asymptotic parameter tending to $\infty$ to mean $A(\lambda)=B(\lambda)+o(B(\lambda))$ as $\lambda \rightarrow \infty$. Since $\mathbf{K}$ is a compact manifold, $-\Delta_{\mathbf{K}}+\xi R_{\gamma}$ has compact resolvent. We denote $\left(\mu_{n}\right)_{n \in \mathbb{N}}$ the eigenvalues of this operator and we assume that $\mu_{n} \leqslant \mu_{n+1}$. We introduce

$$
N:=\min \left\{n \in \mathbb{N} ; \delta<\mu_{n}\right\} .
$$

We introduce a Hilbert basis $\left(\phi_{n}(\mathbf{x})\right)_{n \in \mathbb{N}}$ of $L^{2}(\mathbf{K})$, composed of eigenfunctions, $-\Delta_{\mathbf{K}} \phi_{n}+\xi R_{\gamma} \phi_{n}=$ $\mu_{n} \phi_{n}$. Then the vectors $\Phi_{\text {pos }(n e g), n}^{ \pm}:=2^{-\frac{1}{2}}\left(\left(\mu_{n}+V_{ \pm}\right)^{-\frac{1}{4}} \phi_{n},+(-) i\left(\mu_{n}+V_{ \pm}\right)^{\frac{1}{4}} \phi_{n}\right), n \geqslant N$, form a Hilbert basis $\mathcal{B}_{\text {pos(neg) }}^{ \pm}$of $\left(X_{\text {pos }(n e g)}^{ \pm},\|\cdot\|_{ \pm}\right)$, and $\mathcal{B}_{\text {pos }}^{ \pm} \cup \mathcal{B}_{\text {neg }}^{ \pm}$is a Hilbert basis of $\left(X,\|\cdot\|_{ \pm}\right)$. To establish the theorem, we have to prove that

$$
\sum_{n=N}^{\infty}\left\|P_{n e g(p o s)}^{+} S \Phi_{\text {pos }(n e g), n}^{-}\right\|_{+}^{2}<\infty .
$$

In the sequel, we consider only $P_{\text {neg }}^{+} S P_{\text {pos }}^{-}$, the case of $P_{\text {pos }}^{+} S P_{\text {neg }}^{-}$being analogous. We denote $\varphi_{n}(\tau, \mathbf{x})$ the solution of (I.7) with initial data

$$
\left(\varphi_{n}(0, .), \partial_{\tau} \varphi_{n}(0, .)\right)=W_{-}^{-1} \Phi_{p o s, n}^{-}
$$

that is to say, either

$$
\left(\varphi\left(\tau_{-}, \mathbf{x}\right), \partial_{\tau} \varphi_{n}\left(\tau_{-}, \mathbf{x}\right)\right)=\Phi_{p o s, n}^{-}(\mathbf{x}) \text { if } \tau_{-}>-\infty
$$

or

$$
\lim _{\tau \rightarrow \tau_{-}}\left\|\left(\varphi_{n}(\tau), \partial_{\tau} \varphi_{n}(\tau)\right)-e^{+i \tau \sqrt{\mu_{n}+V_{-}}} \Phi_{\text {pos }, n}^{-}\right\|_{X}=0 \text { if } \tau_{-}=-\infty .
$$

Since the perturbation $V$ does not depend on $\mathbf{x}$, the dynamics of (I.7) does not mix the modes $\phi_{n}$. Then $\varphi_{n}(\tau, \mathbf{x})$ has the form

$$
\varphi_{n}(\tau, \mathbf{x})=\psi_{n}(\tau) \phi_{n}(\mathbf{x})
$$

where $\psi_{n}$ is solution of the differential equation

$$
\psi^{\prime \prime}+\mu_{n} \psi+V(\tau) \psi=0, \quad \tau \in\left(\tau_{-}, \tau_{+}\right),
$$

and satisfies the incoming data

$$
\begin{aligned}
& \text { if } \tau_{-}>-\infty, \quad \psi_{n}\left(\tau_{-}\right)=2^{-\frac{1}{2}}\left(\mu_{n}+V_{-}\right)^{-\frac{1}{4}}, \quad \psi_{n}^{\prime}\left(\tau_{-}\right)=2^{-\frac{1}{2}} i\left(\mu_{n}+V_{-}\right)^{\frac{1}{4}}, \\
& \text { if } \tau_{-}=-\infty, \quad \psi_{n}(\tau) \sim 2^{-\frac{1}{2}}\left(\mu_{n}+V_{-}\right)^{-\frac{1}{4}} e^{i \tau \sqrt{\mu_{n}+V_{-}}}, \tau \rightarrow-\infty .
\end{aligned}
$$

We put

$$
S \Phi_{\text {pos }, n}^{-}=\left(\varphi_{0, n}^{+}, \varphi_{1, n}^{+}\right)=\left(\lambda_{0, n} \phi_{n}, \lambda_{1, n} \phi_{n}\right)=\alpha_{n} \Phi_{\text {pos }, n}^{+}+\beta_{n} \Phi_{n e g, n}^{+}, \quad \lambda_{j, n}, \alpha_{n}, \beta_{n} \in \mathbb{C} .
$$

Then (VII.14) is equivalent to

$$
\sum_{n=N}^{\infty}\left|\beta_{n}\right|^{2}<\infty
$$

On the one hand we have

$$
\alpha_{n}=\frac{1}{2}\left(\lambda_{0, n}+i\left(\mu_{n}+V_{+}\right)^{-\frac{1}{2}} \lambda_{1, n}\right), \beta_{n}=\frac{1}{2}\left(-i\left(\mu_{n}+V_{+}\right)^{\frac{1}{2}} \lambda_{0, n}+\lambda_{1, n}\right),
$$

and on the other hand

$$
\begin{gathered}
\text { if } \tau_{+}<\infty, \psi_{n}\left(\tau_{+}\right)=\lambda_{0, n}, \psi_{n}^{\prime}\left(\tau_{+}\right)=\lambda_{1, n} \\
\text { if } \tau_{+}=\infty, \quad \psi_{n}(\tau) \sim 2^{-\frac{1}{2}}\left(\mu_{n}+V_{+}\right)^{-\frac{1}{4}}\left(\alpha_{n} e^{i \tau} \sqrt{\mu_{n}+V_{+}}+\beta_{n} e^{-i \tau \sqrt{\mu_{n}+V_{+}}}\right), \tau \rightarrow \infty .
\end{gathered}
$$

Now the strategy of the proof consists in proving the following key estimate

$$
\beta_{n}=O\left(\mu_{n}^{-\frac{s}{2}}\right), s>d / 2 \text {. }
$$


Therefore to have (VII.18) it is sufficient to establish that for some $N_{0} \geqslant N$

$$
\sum_{n=N_{0}}^{\infty} \mu_{n}^{-s}<\infty
$$

We take $M=|\xi|\left\|R_{\gamma}\right\|_{L^{\infty}}+1$. Then (VII.20) means that $\left(-\Delta_{\mathbf{K}}+\xi R_{\gamma}+M\right)^{-1}$ is a sth Shatten class operator. We write $\left(-\Delta_{\mathbf{K}}+\xi R_{\gamma}+M\right)^{-1}=\left(I d+\left(-\Delta_{\mathbf{K}}+M\right)^{-1} \xi R_{\gamma}\right)^{-1}\left(-\Delta_{\mathbf{K}}+M\right)^{-1}$. Since the space of the $s$ th Shatten class operators is an ideal of the bounded operators, it is sufficient to prove that $\left(-\Delta_{\mathbf{K}}+M\right)^{-1}$ is a sth Shatten class operator. This property is equivalent to

$$
\zeta(s)<\infty, \zeta(s):=\sum_{\lambda_{n} \in \sigma\left(-\Delta_{\mathbf{K}}\right) \backslash\{0\}} \lambda_{n}^{-s} .
$$

We conclude by invoking a famous result of Minakshisundaram and Pleijel on the Zeta Function of a Laplacian on a $d$-dimensional compact manifold (see e.g. Theorem 5.2 in [56]) that states that the series $\zeta(s)$ converges if $s>\frac{d}{2}$. In the sequel, we establish (VII.19) with $s=l(d)+1$, by distinguishing the various cases according to $\tau_{ \pm}$is finite or not, and $V_{-}=V_{+}$or not.

(1) We begin with the case $\tau_{-}=-\infty, \tau_{+}=\infty, V_{-}=V_{+}$. First, we consider the assumptions assuring that $V_{-}=V_{+}=0$, hence we assume that $\xi=\frac{d-1}{4 d}$ and $\eta_{0}^{ \pm} \geqslant 1$, or $\xi \neq \frac{d-1}{4 d}$ and $\eta_{0}^{ \pm}>1$. Then $V \in C^{l(d)}(\mathbb{R}), V_{-}=V_{+}=0$ and $\frac{d^{k}}{d \tau^{k}} V \in L^{1} \cap L^{\infty}$ for $k \leqslant l(d)$. Since $V$ is integrable and $\mu_{n} \neq 0$, the integral equation

$$
\psi(\tau)=e^{i \sqrt{\mu_{n}} \tau}+\int_{-\infty}^{\tau} \frac{\sin \left(\sqrt{\mu_{n}}(\tau-\sigma)\right)}{\sqrt{\mu_{n}}} V(\sigma) \psi(\sigma) d \sigma
$$

has a unique solution $\psi=\sqrt{2} \mu_{n}^{\frac{1}{4}} \psi_{n}$ that satisfies

$$
\psi(\tau) \sim \alpha_{n} e^{i \sqrt{\mu_{n}} \tau}+\beta_{n} e^{-i \sqrt{\mu_{n}} \tau}, \quad \tau \rightarrow+\infty .
$$

We approximate $\psi$ with a Liouville-Green function. Following [43] we define recursively functions $A_{k}(\tau), 0 \leqslant k \leqslant l(d)+1$, by the relations

$$
A_{0}=1, \quad A_{k+1}(\tau)=-\frac{1}{2} A_{k}^{\prime}(\tau)-\frac{1}{2} \int_{0}^{\tau} V(\sigma) A_{k}(\sigma) d \sigma .
$$

Then Theorem 6 of [43] assures that for all $n \geqslant N_{0}, N_{0}$ large enough, there exists a solution $w\left(\mu_{n} ;\right.$.) of (VII.15) satisfying

$$
w\left(\mu_{n} ; \tau\right)=e^{i \sqrt{\mu_{n}} \tau} \sum_{k=0}^{l(d)} \frac{A_{k}(\tau)}{\left(i \sqrt{\mu_{n}}\right)^{k}}+\epsilon\left(\mu_{n} ; \tau\right),
$$

with

$$
\forall n \geqslant N_{0}, \quad \forall \tau \in \mathbb{R}, \quad\left|\epsilon\left(\mu_{n} ; \tau\right)\right|+\mu_{n}^{\frac{1}{2}}\left|\partial_{\tau} \epsilon\left(\mu_{n} ; \tau\right)\right| \leqslant C \mu_{n}^{-\frac{l(d)+1}{2}} \int_{0}^{\infty}\left|A_{l(d)+1}^{\prime}(\sigma)\right| d \sigma .
$$

We easily prove by recurrence that for $1 \leqslant p \leqslant l(d)+1$

$$
A_{p}^{\prime}(\tau)=\sum_{k=0}^{p-1}\left(-\frac{1}{2}\right)^{k+1} \frac{d^{k}}{d \tau^{k}}\left(V A_{p-1-k}\right)(\tau),
$$

and it follows by recurrence that

$$
k \leqslant l(d)+1, \quad A_{k} \in C^{l(d)+2-k}(\mathbb{R}), \quad 1 \leqslant p \leqslant l(d)+2-k, \frac{d^{p} A_{k}}{d \tau^{p}} \in L^{1} \cap L^{\infty}(\mathbb{R}),
$$


In particular $A_{l(d)+1}^{\prime} \in L^{1}(\mathbb{R})$, hence (VII.22) implies

$$
\forall n>N_{0}, \forall \tau \in \mathbb{R},\left|\epsilon\left(\mu_{n} ; \tau\right)\right| \leqslant C \mu_{n}^{-\frac{l(d)+1}{2}},
$$

We also deduce that for any $k \leqslant l(d), A_{k}(\tau)$ has finite limits as $\tau \rightarrow \pm \infty$. Moreover, since $V \in L^{1}$ there exists $\alpha^{ \pm}\left(\mu_{n}\right), \beta^{ \pm}\left(\mu_{n}\right) \in \mathbb{C}$ such that

$$
w\left(\mu_{n} ; \tau\right) \sim \alpha^{ \pm}\left(\mu_{n}\right) e^{i \sqrt{\mu_{n}} \tau}+\beta^{ \pm}\left(\mu_{n}\right) e^{-i \sqrt{\mu_{n}} \tau}, \quad \tau \rightarrow \pm \infty .
$$

We deduce from (VII.25) that for $n \geqslant N_{0}$,

$$
\left|\beta^{ \pm}\left(\mu_{n}\right)\right| \leqslant C \mu_{n}^{-\frac{l(d)+1}{2}} .
$$

On the other hand we have

$$
w\left(\mu_{n} ; \tau\right)=\cos \left(\tau \sqrt{\mu_{n}}\right) w\left(\mu_{n} ; 0\right)+\frac{\sin \left(\tau \sqrt{\mu_{n}}\right)}{\sqrt{\mu_{n}}} \partial_{\tau} w\left(\mu_{n} ; 0\right)+\int_{0}^{\tau} \frac{\sin \left((\tau-\sigma) \sqrt{\mu_{n}}\right)}{\sqrt{\mu_{n}}} V(\sigma) w\left(\mu_{n} ; \sigma\right) d \sigma,
$$

hence we obtain

$$
\alpha^{ \pm}\left(\mu_{n}\right)=\frac{1}{2}\left(w\left(\mu_{n} ; 0\right)+\frac{\partial_{\tau} w\left(\mu_{n} ; 0\right)}{i \sqrt{\mu_{n}}}-\frac{1}{2 i \sqrt{\mu_{n}}} \int_{0}^{ \pm \infty} e^{-i \sqrt{\mu_{n}} \sigma} V(\sigma) w\left(\mu_{n} ; \sigma\right) d \sigma\right) .
$$

Since $A_{0}=1$ we deduce from (VII.21) and (VII.25) that

$$
\alpha^{ \pm}\left(\mu_{n}\right)=1+O\left(\mu_{n}^{-\frac{1}{2}}\right) .
$$

Expressing $\psi$ on the basis $\left\{w\left(\mu_{n} ;.\right), w^{*}\left(\mu_{n} ;.\right)\right\}$ we obtain

$$
\beta_{n}=\frac{\alpha^{-*}\left(\mu_{n}\right) \beta^{+}\left(\mu_{n}\right)-\alpha^{+*}\left(\mu_{n}\right) \beta^{-}\left(\mu_{n}\right)}{\left|\alpha^{-}\left(\mu_{n}\right)\right|^{2}-\left|\beta^{-}\left(\mu_{n}\right)\right|^{2}},
$$

hence we deduce that

$$
\beta_{n}=O\left(\mu_{n}^{-\frac{l(d)+1}{2}}\right),
$$

and we conclude that (VII.19) holds with $s=l(d)+1$. The case (VII.10) is similar since $V_{-}=V_{+}$. We can employ the same method replacing $\mu_{n}$ by $\mu_{n}+V_{ \pm}$and $V(\tau)$ by $V(\tau)-V_{ \pm}$.

(2) Now we consider the case $\tau_{-}=-\infty, \tau_{+}=\infty$ and possibly $V_{-} \neq V_{+}$, i.e. we assume (VII.11). If $\eta_{0}^{-}=\eta_{0}^{+}=1$ (and $c_{0}^{-} \neq c_{0}^{+}$), we have $\int_{0}^{ \pm}\left|V(\tau)-V_{ \pm}\right|(1+|\tau|) d \tau<\infty$ and we can invoke the Lemma 1.4 (iv) of [16] that implies that (VII.19) holds with $s=3$. If one $\eta_{0}^{ \pm}>1, V(\tau)-V_{ \pm}$ decays as $\tau^{-1}$ and we cannot applied the known results. We consider an integer $N_{0} \geqslant N$ such that

$$
\forall \tau \in\left(\tau_{-}, \tau_{+}\right), \quad \mu_{N_{0}}>0, \quad \mu_{N_{0}}+V(\tau) \geqslant 1 .
$$

For any $n \geqslant N_{0}$ we take the Liouville-Green solution of (VII.15) introduced by Olver (Theorem 4 in [43]) that satisfies

$$
\begin{array}{r}
w\left(\mu_{n} ; \tau\right)=2^{-\frac{1}{2}}\left(\mu_{n}+V(\tau)\right)^{-\frac{1}{4}} \exp \left(i \int_{0}^{\tau}\left(\mu_{n}+V(\sigma)\right)^{\frac{1}{2}} d \sigma\right)\left(1+\epsilon_{n}(\tau)\right), \\
\left|\epsilon_{n}(\tau)\right|, \mu_{n}^{-\frac{1}{2}}\left|\epsilon_{n}^{\prime}(\tau)\right| \leqslant \exp \left(\left|\int_{0}^{\tau}\left(\mu_{n}+V(\sigma)\right)^{-\frac{1}{4}}\right| \frac{d^{2}}{d \sigma^{2}}\left(\mu_{n}+V(\sigma)\right)^{-\frac{1}{4}}|d \sigma|\right)-1 .
\end{array}
$$

Since $V^{\prime} \in L^{2}\left(\tau_{-}, \tau_{+}\right), V^{\prime \prime} \in L^{1}\left(\tau_{-}, \tau_{+}\right)$we get

$$
\sup _{\tau \in\left(\tau_{-}, \tau_{+}\right)}\left(\left|\epsilon_{n}(\tau)\right|+\mu_{n}^{-\frac{1}{2}}\left|\epsilon_{n}^{\prime}(\tau)\right|\right) \leqslant C \mu_{n}^{-\frac{3}{2}},
$$


As a consequence of $\int_{0}^{\tau_{ \pm}}\left|V(\tau)-V_{ \pm}\right| d \tau<\infty$, there exists $\alpha^{ \pm}\left(\mu_{n}\right), \beta^{ \pm}\left(\mu_{n}\right) \in \mathbb{C}$ such that

$$
w\left(\mu_{n} ; \tau\right) \sim 2^{-\frac{1}{2}}\left(\mu_{n}+V_{ \pm}\right)^{-\frac{1}{4}}\left(\alpha^{ \pm}\left(\mu_{n}\right) e^{i \tau \sqrt{\mu_{n}+V_{ \pm}}}+\beta^{ \pm}\left(\mu_{n}\right) e^{-i \tau \sqrt{\mu_{n}+V_{ \pm}}}\right), \quad \tau \rightarrow \pm \infty .
$$

Using the fact that

$$
\begin{gathered}
\alpha^{ \pm}\left(\mu_{n}\right)=2^{\frac{1}{2}}\left(\mu_{n}+V_{ \pm}\right)^{\frac{1}{4}} \lim _{\tau \rightarrow \pm \infty} \frac{1}{\tau} \int_{0}^{\tau} e^{-i \sigma \sqrt{\mu_{n}+V_{ \pm}}} w\left(\mu_{n} ; \sigma\right) d \sigma, \\
\beta^{ \pm}\left(\mu_{n}\right)=2^{\frac{1}{2}}\left(\mu_{n}+V_{ \pm}\right)^{\frac{1}{4}} \lim _{\tau \rightarrow \pm \infty} \frac{1}{\tau} \int_{0}^{\tau} e^{i \sigma \sqrt{\mu_{n}+V_{ \pm}}} w\left(\mu_{n} ; \sigma\right) d \sigma,
\end{gathered}
$$

we get

$$
\begin{gathered}
\alpha^{ \pm}\left(\mu_{n}\right)=\exp \left[i \int_{0}^{ \pm \infty} \frac{V(\tau)-V_{ \pm}}{\left(\mu_{n}+V(\tau)\right)^{\frac{1}{2}}+\left(\mu_{n}+V_{ \pm}\right)^{\frac{1}{2}}} d \tau\right]+O\left(\mu_{n}^{-\frac{3}{2}}\right), \\
\beta^{ \pm}\left(\mu_{n}\right)=O\left(\mu_{n}^{-\frac{3}{2}}\right) .
\end{gathered}
$$

Now we express $\psi_{n}$ on the basis $\left\{w\left(\mu_{n} ;.\right), w^{*}\left(\mu_{n} ;.\right)\right\}$ by putting $\psi_{n}(\tau)=A w\left(\mu_{n} ; \tau\right)+B w^{*}\left(\mu_{n} ; \tau\right)$, $A, B \in \mathbb{C}$. We have

$$
\begin{gathered}
1=A \alpha^{-}\left(\mu_{n}\right)+B \beta^{-*}\left(\mu_{n}\right), \quad 0=A \beta^{-}\left(\mu_{n}\right)+B \alpha^{-*}\left(\mu_{n}\right), \\
\alpha_{n}=A \alpha^{+}\left(\mu_{n}\right)+B \beta^{+*}\left(\mu_{n}\right), \quad \beta_{n}=A \beta^{+}\left(\mu_{n}\right)+B \alpha^{+*}\left(\mu_{n}\right),
\end{gathered}
$$

and $\beta_{n}$ is given by (VII) again. Hence we obtain from (VII.30) and (VII.31) that (VII.19) holds with $s=3$.

(3) Now we consider the cases for which $\tau_{+}$and $\tau_{-}$are finite and $V_{-} \neq V_{+}$. From the assumption (VII.12) with $\eta_{0}^{-} \in(1 / 3,1)$, we have $V \in C^{2}\left(\tau_{-}, \tau_{+}\right) \cap C^{0}\left(\left[\tau_{-}, \tau_{+}\right]\right), V^{\prime} \in L^{2}\left(\tau_{-}, \tau_{+}\right), V^{\prime \prime} \in L^{1}\left(\tau_{-}, \tau_{+}\right)$. We denote $\psi_{+}$the solution of (VII.15) with data

$$
\psi_{+}\left(\tau_{+}\right)=2^{-\frac{1}{2}}\left(\mu_{n}+V_{+}\right)^{-\frac{1}{4}}, \psi_{+}^{\prime}\left(\tau_{+}\right)=2^{-\frac{1}{2}} i\left(\mu_{n}+V_{+}\right)^{\frac{1}{4}} .
$$

$\psi_{+}$and $\psi_{+}^{*}$ are linearly independent and we have

$$
\psi_{n}(\tau)=\alpha_{n} \psi_{+}(\tau)+\beta_{n} \psi_{+}^{*}(\tau) .
$$

Now we choose the two complex-conjugate linearly independent solutions $w, w^{*}$ defined by (VII.28) and we express $\psi_{n}$ and $\psi_{+}$as:

$$
\psi_{n}(\tau)=A w(\tau)+B w^{*}(\tau), w(\tau)=\alpha \psi_{+}(\tau)+\beta \psi_{+}^{*}(\tau), \quad A, B, \alpha, \beta \in \mathbb{C} .
$$

We deduce that

$$
\alpha_{n}=A \alpha+B \beta^{*}, \quad \beta_{n}=A \beta+B \alpha^{*} .
$$

Matching with the data (VII.16) and (VII.32), we have

$$
\begin{gathered}
A=2^{-\frac{1}{2}}\left(\mu_{n}+V_{-}\right)^{-\frac{1}{4}} \frac{\left(w^{\prime}\left(\tau_{-}\right)+i\left(\mu_{n}+V_{-}\right)^{\frac{1}{2}} w\left(\tau_{-}\right)\right)^{*}}{\left[w, w^{*}\right]}, \\
B=-2^{-\frac{1}{2}}\left(\mu_{n}+V_{-}\right)^{-\frac{1}{4}} \frac{w^{\prime}\left(\tau_{-}\right)-i\left(\mu_{n}+V_{-}\right)^{\frac{1}{2}} w\left(\tau_{-}\right)}{\left[w, w^{*}\right]}, \\
\alpha=-2^{-\frac{1}{2}} i\left(\mu_{n}+V_{+}\right)^{-\frac{1}{4}}\left(w^{\prime}\left(\tau_{+}\right)+i\left(\mu_{n}+V_{+}\right)^{\frac{1}{2}} w\left(\tau_{+}\right)\right), \\
\beta=2^{-\frac{1}{2}} i\left(\mu_{n}+V_{+}\right)^{-\frac{1}{4}}\left(w^{\prime}\left(\tau_{+}\right)-i\left(\mu_{n}+V_{+}\right)^{\frac{1}{2}} w\left(\tau_{+}\right),\right.
\end{gathered}
$$


where $[f, g]$ is the Wronskian, $[f, g]:=f g^{\prime}-f^{\prime} g$. Since $V^{\prime} \in L^{2}\left(\tau_{-}, \tau_{+}\right), V^{\prime \prime} \in L^{1}\left(\tau_{-}, \tau_{+}\right)$we have (VII.29) again, hence $\left[w, w^{*}\right] \sim 2 i, n \rightarrow \infty$, and $A, \alpha=O(1)$ and $B, \beta=O\left(\mu_{n}^{-\frac{3}{2}}\right)$. Therefore we obtain the key estimate (VII.19) with $s=3$. We conclude that (VII.18) holds if $d \leqslant 5$.

(4) Now we consider the cases for which $\tau_{+}$and $\tau_{-}$are finite and $V_{-}=V_{+}=0$, i.e. $\xi=\frac{d-1}{4 d}$, $\eta_{0}^{ \pm} \in\left(\frac{l-1}{l+1}, 1\right)$. We apply the method of the previous case (3) but we choose another LiouvilleGreen function $w$ by taking (VII.21). We remark that $\frac{d^{k} V}{d \tau^{k}}\left(\tau_{ \pm}\right)=0$ for $0 \leqslant k \leqslant l(d)-1$ and $\frac{d^{l(d)} V}{d \tau^{l(d)}} \in L^{1}\left(\tau_{-}, \tau_{+}\right)$. We deduce that $A_{k}^{\prime}\left(\tau_{ \pm}\right)=0$ for $0 \leqslant k \leqslant l(d)$ and $A_{l(d)+1}^{\prime} \in L^{1}\left(\tau_{-}, \tau_{+}\right)$. Then we have

$$
w^{\prime}\left(\tau_{ \pm}\right)-i \sqrt{\mu_{n}} w\left(\tau_{ \pm}\right)=O\left(\mu_{n}^{-\frac{l(d)}{2}}\right)
$$

and we obtain $\left[w, w^{*}\right] \sim 2 i \sqrt{\mu_{n}}, n \rightarrow \infty, A=O\left(\mu_{n}^{-\frac{1}{4}}\right), \alpha=O\left(\mu_{n}^{\frac{1}{4}}\right), B=O\left(\mu_{n}^{-\frac{1}{4}-\frac{l(d)+1}{2}}\right)$, $\beta=O\left(\mu_{n}^{-\frac{1}{4}-\frac{l(d)}{2}}\right)$, therefore (VII.19) holds with $s=\frac{l(d)+1}{2}$.

(5) We consider the case $\tau_{-}=-\infty, \tau_{+}<\infty, V_{-}=V_{+}=0$, that occurs if $\xi=\frac{d-1}{4 d}, \eta_{0}^{-} \geqslant 1$, $\eta_{0}^{+} \in\left(\frac{l-1}{l+1}, 1\right)$. We have $\frac{d^{k} V}{d \tau^{k}}\left(\tau_{+}\right)=0$ for $0 \leqslant k \leqslant l(d)-1$ and $\frac{d^{l(d)} V}{d \tau^{l(d)}} \in L^{1}\left(-\infty, \tau_{+}\right)$. We deduce that $A_{k}^{\prime}\left(\tau_{+}\right)=0$ for $0 \leqslant k \leqslant l(d)$ and $A_{l(d)+1}^{\prime} \in L^{1}\left(-\infty, \tau_{+}\right)$. We employ the Liouville-Green function (VII.21) that satisfies

$$
w^{\prime}\left(\tau_{+}\right)+i \sqrt{\mu_{n}} w\left(\tau_{+}\right)=O\left(\mu_{n}^{-\frac{l(d)}{2}}\right),
$$

and $\left[w, w^{*}\right] \sim 2 i \sqrt{\mu_{n}}, n \rightarrow \infty$. There exist $\alpha^{-}\left(\mu_{n}\right), \beta^{-}\left(\mu_{n}\right) \in \mathbb{C}$ such that

$$
w\left(\mu_{n} ; \tau\right) \sim \alpha^{-}\left(\mu_{n}\right) e^{i \sqrt{\mu_{n}} \tau}+\beta^{-}\left(\mu_{n}\right) e^{-i \sqrt{\mu_{n}} \tau}, \quad \tau \rightarrow-\infty,
$$

and like in the case (1), they satisfy

$$
\alpha^{-}\left(\mu_{n}\right)=1+O\left(\mu_{n}^{-\frac{1}{2}}\right), \beta^{-}\left(\mu_{n}\right)=O\left(\mu_{n}^{-\frac{l(d)+1}{2}}\right) .
$$

We use (VII.33) and VII.34) in which

$$
\begin{gathered}
A=2^{-\frac{1}{2}} \mu_{n}^{-\frac{1}{4}} \frac{\left(\alpha^{-}\left(\mu_{n}\right)\right)^{*}}{\left|\alpha^{-}\left(\mu_{n}\right)\right|^{2}-\left|\beta^{-}\left(\mu_{n}\right)\right|^{2}} \sim 2^{-\frac{1}{2}} \mu_{n}^{-\frac{1}{4}}, n \rightarrow \infty, \\
B=2^{-\frac{1}{2}} \mu_{n}^{-\frac{1}{4}} \beta^{-}\left(\mu_{n}\right)=O\left(\mu_{n}^{-\frac{1}{4}-\frac{l(d)+1}{2}}\right), \\
\alpha \sim 2^{\frac{1}{2}} \mu_{n}^{\frac{1}{4}}, n \rightarrow \infty, \beta=O\left(\mu_{n}^{-\frac{1}{4}-\frac{l(d)}{2}}\right) .
\end{gathered}
$$

We conclude that we have $\beta_{n}=O\left(\mu_{n}^{-\frac{l(d)+1}{2}}\right)$.

(6) We consider the case $\tau_{-}=-\infty, \tau_{+}<\infty, V_{-} \neq V_{+}$, that occurs if $d \leqslant 5, \eta_{0}^{-} \geqslant 1, \eta_{0}^{+}=0$, with $\eta_{1}^{+}>1$ and $l=2$ if $\xi=\frac{d-1}{4 d}$, or $\eta_{1}^{+}>3$ and $l=4$ if $\xi \neq \frac{d-1}{4 d}$. With these hypotheses, $V \in C^{2}\left(-\infty, \tau_{+}\right) \cap C^{0}\left(\left(-\infty, \tau_{+}\right]\right), V^{\prime} \in L^{2}\left(-\infty, \tau_{+}\right), V^{\prime \prime} \in L^{1}\left(-\infty, \tau_{+}\right), \int_{-\infty}^{0}\left|V(\tau)-V_{-}\right| d \tau<\infty$. We adapt the method employed for the case (2). We take the Liouville-Green function $w\left(\mu_{n}, \tau\right)$ defined by (VII.28) that satisfies (VII.29) again. We have

$$
w\left(\mu_{n} ; \tau\right) \sim 2^{-\frac{1}{2}}\left(\mu_{n}+V_{-}\right)^{-\frac{1}{4}}\left(\alpha^{-}\left(\mu_{n}\right) e^{i \tau \sqrt{\mu_{n}+V_{-}}}+\beta^{-}\left(\mu_{n}\right) e^{-i \tau \sqrt{\mu_{n}+V_{-}}}\right), \quad \tau \rightarrow-\infty,
$$




$$
\begin{gathered}
\alpha^{-}\left(\mu_{n}\right)=\exp \left[i \int_{0}^{-\infty} \frac{V(\tau)-V_{-}}{\left(\mu_{n}+V(\tau)\right)^{\frac{1}{2}}+\left(\mu_{n}+V_{-}\right)^{\frac{1}{2}}} d \tau\right]+O\left(\mu_{n}^{-\frac{3}{2}}\right), \beta^{-}\left(\mu_{n}\right)=O\left(\mu_{n}^{-\frac{3}{2}}\right), \\
w^{\prime}\left(\tau_{+}\right)+i \sqrt{\mu_{n}} w\left(\tau_{+}\right)=O\left(\mu_{n}^{\frac{1}{4}}\right), w^{\prime}\left(\tau_{+}\right)-i \sqrt{\mu_{n}} w\left(\tau_{+}\right)=O\left(\mu_{n}^{-\frac{5}{4}}\right) .
\end{gathered}
$$

Using (VII.33) and VII.34), we have $A, \alpha=O(1), B, \beta=O\left(\mu_{n}^{-\frac{3}{2}}\right)$ and we conclude that $\beta_{n}=O\left(\mu_{n}^{-\frac{3}{2}}\right)$.

(7) The last case concerns $\tau_{-}>-\infty, \tau_{+}=\infty$ that occurs for $\eta_{0}^{-} \in\left(\frac{l-1}{l+1}, 1\right), \eta_{0}^{+} \geqslant 1$. By a time reversing, it is equivalent to the case (5). Finally we have investigated all the situations and the proof of the theorem is complete.

Q.E.D.

\section{CONCLUSiON}

In this work we have carried out a complete description of the asymptotics of the solutions of the linear Klein-Gordon equation on a FLRW universe beginning with a Big Bang and ending with a Big Crunch, a Big Rip, or a Sudden Singularity. In these cases, the dynamics is defined by a scalar equation with a time-dependent mass that can be zero or infinite at these singularities. We also have obtained similar results for the semilinear Klein-Gordon equation in the simple case of subcritical exponents. The fundamental problem of the general non-linearity $|u|^{p-1} u$ is open. This case is much more difficult and certainly refined tools of harmonic analysis will be necessary, such as Strichartz estimates or the $L^{p}-L^{q}$ continuity of the propagator. Due to the time dependence of the coefficients and their singularity at the Big Bang/Crunch/Rip, these properties are certainly hard to get. Nevertheless, we note that in the case of the FLRW universes without singularity or the De Sitter space-time, similar results have be obtained [29, [30], 31], [40]. Finally we have showed that the number of cosmological particle creation is finite under rather general assumptions on the initial Big Bang and the final Big Crunch or Big Brake. The concept of particles in the dynamical universes is rather ambiguous. It would be interesting to pursue the investigation of the quantum fields near a general time singularity, by the study of fiducial quantum states in the spirit of [18] or 32 .

\section{Appendix}

This appendix is devoted to the asymptotics near a time-future singularity, of the scale factor expressed with the conformal time. The scale factor $a(t)$ is a positive function in $C^{l}\left(\left[t_{0}, t_{+}\right)\right)$and we assume that near $t_{+}$we have as $t \rightarrow t_{+}$

$$
\frac{d^{k} a}{d t^{k}}(t)=\frac{d^{k}}{d t^{k}}\left[c_{0}\left(t_{+}-t\right)^{\eta_{0}}+c_{1}\left(t_{+}-t\right)^{\eta_{1}}\right]+o\left(\left(t_{+}-t\right)^{\eta_{1}-k}\right), \quad k \leqslant l
$$

where the coefficients $c_{0}, \eta_{j} \in \mathbb{R}$ satisfy :

$$
c_{0}>0, \eta_{0}<\eta_{1}
$$

The conformal time $\tau$ is defined by

$$
\tau:=\int_{t_{0}}^{t} \frac{1}{a(s)} d s, \quad \tau_{+}:=\int_{t_{0}}^{t_{+}} \frac{1}{a(s)} d s \in(0, \infty],
$$

and the scale factor in this coordinate is expressed as

$$
\alpha(\tau):=a(t) .
$$


We begin by the case $\eta_{0}<1$. Then $\tau_{+}<\infty$ and we write for $t_{+}-t$ small enough

$$
\begin{aligned}
\tau_{+}-\tau & =c_{0}^{-1} \int_{t}^{t_{+}}\left(t_{+}-s\right)^{-\eta_{0}}\left[1-\frac{c_{1}}{c_{0}}\left(t_{+}-s\right)^{\eta_{1}-\eta_{0}}+o\left(\left(t_{+}-s\right)^{\eta_{1}-\eta_{0}}\right)\right] d s \\
& =c_{0}^{-1}\left(1-\eta_{0}\right)^{-1}\left(t_{+}-t\right)^{1-\eta_{0}}\left[1-\frac{c_{1}}{c_{0}} \frac{\left(1-\eta_{0}\right)}{\left(1-2 \eta_{0}+\eta_{1}\right)}\left(t_{+}-t\right)^{\eta_{1}-\eta_{0}}+o\left(\left(t_{+}-t\right)^{\eta_{1}-\eta_{0}}\right)\right] .
\end{aligned}
$$

We deduce that

$$
t_{+}-t=\left[c_{0}\left(1-\eta_{0}\right)\right]^{\frac{1}{1-\eta_{0}}}\left(\tau_{+}-\tau\right)^{\frac{1}{1-\eta_{0}}}\left[1+O\left(\left(\tau_{+}-\tau\right)^{\frac{\eta_{1}-\eta_{0}}{1-\eta_{0}}}\right)\right] .
$$

We conclude that the scale factor satisfies for $k \leqslant l$

$$
\frac{d^{k}}{d \tau^{k}} \alpha(\tau)=\left[c_{0}\left(1-\eta_{0}\right)^{\eta_{0}}\right]^{\frac{1}{1-\eta_{0}}} \frac{d^{k}}{d \tau^{k}}\left(\left(\tau_{+}-\tau\right)^{\frac{\eta_{0}}{1-\eta_{0}}}\right)+O\left(\left(\tau_{+}-\tau\right)^{\frac{\eta_{1}}{1-\eta_{0}}-k}\right), \quad \eta_{0}<1,
$$

and more specifically for $\eta_{0}=0$,

$$
\frac{d^{k}}{d \tau^{k}} \alpha(\tau)=\frac{d^{k}}{d \tau^{k}}\left(c_{0}+c_{1}\left[c_{0}\right]^{\eta_{1}}\left(\tau_{+}-\tau\right)^{\eta_{1}}\right)+o\left(\left(\tau_{+}-\tau\right)^{\eta_{1}-k}\right), \quad k \leqslant l, \quad \eta_{0}=0 .
$$

If $\eta_{0} \geqslant 1$, then $\tau_{+}=+\infty$. First we consider the case $\eta_{0}=1$ that is particular. We have for $t$ close to $t_{+}$

$$
\begin{aligned}
\tau & =c_{0}^{-1} \int_{t_{0}}^{t} \frac{1}{t_{+}-s}\left[1-\frac{c_{1}}{c_{0}}\left(t_{+}-s\right)^{\eta_{1}-1}+o\left(\left(t_{+}-s\right)^{\eta_{1}-1}\right)\right] d s \\
& =-c_{0}^{-1} \ln \left(t_{+}-t\right)+k_{0}+O\left(\left(t_{+}-t\right)^{\eta_{1}-1}\right), \quad k_{0} \in \mathbb{R} .
\end{aligned}
$$

We deduce that

$$
t_{+}-t=e^{-c_{0}\left(\tau-k_{0}\right)}+o\left(e^{-c_{0} \tau}\right)
$$

hence

$$
\frac{d^{k}}{d \tau^{k}} \alpha(\tau)=c_{0} e^{c_{0} k_{0}} \frac{d^{k}}{d \tau^{k}}\left(e^{-c_{0} \tau}\right)+o\left(e^{-c_{0} \tau}\right), \quad k \leqslant l, \quad \eta_{0}=1 .
$$

Finally we consider the case $\eta_{0}>1$. We write

$$
\begin{aligned}
\tau & =c_{0}^{-1} \int_{t_{0}}^{t}\left(t_{+}-s\right)^{-\eta_{0}}\left[1-\frac{c_{1}}{c_{0}}\left(t_{+}-s\right)^{\eta_{1}-\eta_{0}}+o\left(\left(t_{+}-s\right)^{\eta_{1}-\eta_{0}}\right)\right] d s \\
& =\frac{1}{c_{0}\left(\eta_{0}-1\right)}\left(t_{+}-t\right)^{1-\eta_{0}}+o\left(\left(t_{+}-t\right)^{1-\eta_{0}}\right)
\end{aligned}
$$

hence

We conclude that

$$
\left(t_{+}-t\right)=\left[c_{0}\left(\eta_{0}-1\right) \tau\right]^{-\frac{1}{\eta_{0}-1}}+o\left(\tau^{-\frac{1}{\eta_{0}-1}}\right) .
$$

$$
\frac{d^{k}}{d \tau^{k}} \alpha(\tau)=c_{0}^{\frac{1}{1-\eta_{0}}}\left(\eta_{0}-1\right)^{\frac{\eta_{0}}{1-\eta_{0}}} \frac{d^{k}}{d \tau^{k}}\left(\tau^{\frac{\eta_{0}}{1-\eta_{0}}}\right)+o\left(\tau^{\frac{\eta_{0}}{1-\eta_{0}}-k}\right), \quad k \leqslant l, \quad \eta_{0}>1
$$

\section{REFERENCES}

[1] A. Alho, G. Fournodavlos, A. T. Franzen, The wave equation near flat Friedmann-Lemaître-Robertson-Walker and Kasner Big Bang singularities, preprint (2018), arXiv:1805.12558.

[2] P. T. Allen, A. D. Rendall, Asymptotics of linearized cosmological perturbations, J. Hyperbolic Differ. Equ. 7 (2010), no. 2, 255-277.

[3] L. Andersson, A. D. Rendall, Quiescent cosmological singularities, Commun. Math. Phys. 218 (2001), 479-511.

[4] A. Bachelot, A. Bachelot-Motet, Waves on accelerating dodecahedral universes, Class. Quantum Grav. 34 (2017), no. 5, 055010, 39 pp.

[5] J. D. Barrow, A. B. Batista, G. Dito, J. C. Fabris, M. J. S. Houndjo, Sudden singularities survive massive quantum particle production, Phys. Rev. D 84 (2011), 123518. 
[6] J. D. Barrow, S. Cotsakis and A. Tsokaros, A General Sudden Cosmological Singularity, Class. Quantum Grav. 27 (2010), 165017.

[7] J. D. Barrow, A. A. H. Graham, Singular Inflation, Phys. Rev. D 91 (2015), 083513.

[8] J. D. Barrow, S. Z. W. Lip, Classical Stability of Sudden and Big Rip Singularities, Phys. Rev. D 80 (2009), 043518.

[9] F. Beyer, P. G. LeFloch, Second-order hyperbolic Fuchsian systems and applications, Class. Quantum Grav. 27 (2010), 245012.

[10] F. Beyer, P. G. LeFloch, Self-gravitating fluid flows with Gowdy symmetry near cosmological singularities, Comm. Partial Differential Equations 42 (2017), no. 8, 1199-1248.

[11] H. Calderón and W. A. Hiscock, Quantum fields and "big rip" expansion singularities, Class. Quantum Grav. 22 (2005), L23-L26.

[12] R. R. Caldwell, M. Kamionkowski, N. N. Weinberg, Phantom Energy and Cosmic Doomsday, Phys.Rev.Lett. 91 (2003), 071301.

[13] C. Cattoën, M. Visser, Necessary and sufficient conditions for big bangs, bounces, crunches, rips, sudden singularities and extremality events, Class. Quantum Grav. 22 (2005), 4913-4930.

[14] L. P. Chimento, M. G. Richarte, Big brake singularity is accommodated as an exotic quintessence field, Phys. Rev. D 93 (2016), no. 4, 043524.

[15] Y. Choquet-Bruhat, General Relativity and the Einstein Equations (Oxford University Press, 2009).

[16] A. Cohen, T. Kappeler, Scattering and inverse scattering for steplike potentials in the Schrödinger equation, Indiana Univ. Math. J. 34 (1) (1985), 127-180.

[17] T. Damour, M. Henneaux, A. D. Rendall, M. Weaver, Kasner-like behaviour for subcritical Einstein-matter systems, Ann. Henri Poincaré 3 (2002), no. 6, 1049-1111.

[18] A. Degner, R. Verch, Cosmological particle creation in states of low energy, J. Math. Phys. 51 (2010), 022302.

[19] D. Del Santo, T. Kinoshita, M. Reissig, Klein-Gordon Type Equations with a Singular Time-dependent Potential, Rend. Istit. Mat. Univ. Trieste 39 (2007), 141-175.

[20] M. R. Ebert, W. N. Nascimento, A classification for wave models with time-dependent mass and speed of propagation, preprint (2017), arXiv:1710.01212.

[21] M. R. Ebert, M. Reissig, Regularity theory and global existence of small data solutions to semi-linear de Sitter models with power non-linearity, Nonlinear Analysis: Real World Appl. 40 (2018), 14-54.

[22] J. Eichhorn, The Boundedness of Connection Coefficients and their Derivatives, Math. Nachr. 152 (1991), 145158.

[23] J. Eichhorn, The Banach manifold structure of the space of metrics on noncompact manifolds, Differential Geom. Appl. 1 (1991), no. 2, 89-108.

[24] E. Elizalde, S. Nojiri, S.D. Odintsov, Late-time cosmology in (phantom) scalar-tensor theory: dark energy and the cosmic speed-up, Phys. Rev. D, 70 (2004), 043539.

[25] L. Fernández-Jambrina, R. Lazkoz, Classification of cosmological milestones, Phys. Rev. D (3) 74 (2006), no. 6, 064030 .

[26] H. Friedrich, On the existence of n-geodesically complete or future complete solutions of Einstein's field equations with smooth asymptotic structure, Comm. Math. Phys. 107 (1986), no. 4, 587-609.

[27] S. A. Fulling, Aspects of Quantum Field Theory in Curved Space-Time, London Mathematical Society Student Texts, 17 (Cambridge University Press, 1989).

[28] S. A. Fulling, Remarks on positive frequency and Hamiltonians in expanding universes. Gen. Relativity Gravitation 10 (1979), no. 10, 807-824.

[29] A. Galstian, T. Kinoshita, K. Yagdjian, A note on wave equation in Einstein and de Sitter space-time, J. Math. Phys. 51 (2010), no. 5, 052501, 18 pp.

[30] A. Galstian, K. Yagdjian, Global solutions for semilinear Klein-Gordon equations in FLRW spacetimes, Nonlinear Analysis 113 (2015), 339-356.

[31] A. Galstian, K. Yagdjian, Global in time existence of self-interacting scalar field in de Sitter spacetimes, Nonlinear Analysis: Real World Appl. 34 (2017), 110-139.

[32] C. Gérard, O. Oulghazi, M. Wrochna, Hadamard States for the Klein-Gordon Equation on Lorentzian Manifolds of Bounded Geometry, Commun. Math. Phys. 352 (2017), 519-583.

[33] V. Gorini, A. Y. Kamenshchik, U. Moschella, V. Pasquier, Tachyons, scalar fields and cosmology, Phys. Rev. D $69(2004), 123512$

[34] A. A. Grib, Yu. V. Pavlov, Particle creation in the early Universe: achievements and problems Grav. Cosmol. 22 (2016), 107-115.

[35] N. Große, C. Schneider, Sobolev spaces on Riemannian manifolds with bounded geometry: General coordinates and traces, Math. Nach. 286, 16 (2013), 1586-1613.

[36] M. Hadzic, J. Speck, The global future stability of the FLRW so- lutions to the dust-Einstein system with a positive cosmological constant, J. Hyperbolic Differ. Equ. 12 (2015), no. 1, 87-188. 
[37] S. Kichenassamy, A. D. Rendall, Analytic description of singularities in Gowdy spacetimes, Classical Quantum Gravity 15 (1998), no. 5, 1339-1355.

[38] J. Leray, Hyperbolic differential equations (Princeton University Press, 1953).

[39] J-L. Lions, E. Magenes, Non-Homogeneous Boundary Value Problems and Applications, volume I, Die Grundlehren der mathematischen Wissenschaften, 181 (Springer-Verlag, 1972).

[40] M. Nakamura, The Cauchy problem for semi-linear Klein-Gordon equations in de Sitter spacetime, J. Math. Anal. Appl. 410 (2014), 445-454.

[41] F. W. J. Olver, D. W. Lozier, R. F. Boisvert, C. W. Clark, NIST Handbook of Mathematical Functions (Cambridge University Press, 2010).

[42] S. Nojiri, S. D. Odinstov, S. Tsujikawa, Properties of singularities in (phantom) dark energy universe, Phys. Rev. D. 71 (2005), 063004.

[43] F. W. J. Olver, Error bounds for the Liouville-Green (or WKB) approximation, Proc. Cambridge Philos. Soc. 57 (1961) 790-810.

[44] L. E. Parker, D. J. Toms, Quantum Field Theory in Curved Spacetime, Quantized Fields and Gravity, Cambridge Monographs on Mathematical Physics (Cambridge University Press 2009).

[45] M. Reed, B. Simon, Methods of modern mathematical physics III, Scattering Theory (Academic Press, 1975).

[46] A.D. Rendall, Fuchsian analysis of singularities in Gowdy spacetimes beyond analyticity, Classical Quantum Gravity 17 (2000), no. 16, 3305-3316.

[47] H. Ringström, Future stability of the Einstein non-linear scalar field system, Invent. math. 173 (2008), $123-208$.

[48] H. Ringström, Strong cosmic censorship in $T^{3}$-Gowdy spacetimes, Ann. of Math. (2) 170 (2009), no. 3, $1181-1240$.

[49] H. Ringström, On the topology and future stability of the universe, Oxford Mathematical Monographs (Oxford University Press, 2013).

[50] H. Ringström, Linear systems of wave equations on cosmological backgrounds with convergent asymptotics, preprint (2017), arXiv:1707.02803.

[51] H. Ringström, A unified approach to the Klein-Gordon equation on Bianchi backgrounds, preprint (2018), arXiv:1808.00786.

[52] I. Rodnianski, J. Speck, The nonlinear future stability of the FLRW family of solutions to the irrotational Euler-Einstein system with a positive cosmological constant, J. Eur. Math. Soc. 15 (2013), no. 6, $2369-2462$.

[53] I. Rodnianski, J. Speck, A regime of linear stability for the Einstein-scalar field system with applications to nonlinear big bang formation, Ann. of Math. (2) 187 (2018), no. 1, 65-156.

[54] I. Rodnianski, J. Speck, Stable Big Bang formation in near-FLRW solutions to the Einstein-scalar field and Einstein-stiff fluid systems, Selecta Math. (N.S.) 24 (2018), no. 5, 4293-4459.

[55] I. Rodnianski, J. Speck, On the nature of Hawking's incompleteness for the Einstein-vacuum equations: The regime of moderately spatially anisotropic initial data, preprint (2018) arXiv:1804.06825.

[56] S. Rosenberg, The Laplacian on a Riemannian manifold. An introduction to analysis on manifolds, London Mathematical Society Student Texts, 31 (Cambridge University Press 1997).

[57] D. Shale, Linear symmetries of free boson fields, Trans. Amer. Math. Soc. 103 (1962), 149-167.

[58] J. Speck, The nonlinear future stability of the FLRW family of solutions to the Euler-Einstein system with a positive cosmological constant, Selecta Math.18 (2012), no. 3, 633-715.

[59] J. Speck, The Maximal Development of Near-FLRW Data for the Einstein-Scalar Field System with Spatial Topology $S^{3}$, Comm. Math. Phys. 364 (2018), no. 3, 879-979.

[60] W. A. Strauss, On continuity of functions with values in various Banach spaces, Pacific J. Math., 19 (3) (1966), 543-551.

[61] R. S. Strichartz, Analysis of the Laplacian on the complete Riemannian manifold, J. Funct. Anal. 52 (1983), 48-79.

[62] C. Svedberg, Future Stability of the Einstein-Maxwell-Scalar Field System, Ann. Henri Poincaré 12, No. 5, (2011) 849-917.

[63] H. Tanabe, Functional analytic methods for partial differential equations, Pure and Applied Mathematics, 204 (Marcel Dekker, 1997).

Université de Bordeaux, Institut de Mathématiques, UMR CNRS 5251, F-33405 Talence Cedex

E-mail address: alain.bachelot@u-bordeaux.fr 